IZA DP No. 5728

The Economics of Risky Health Behaviors

John Cawley

Christopher J. Ruhm

May 2011 


\title{
The Economics of Risky Health Behaviors
}

\author{
John Cawley \\ Cornell University \\ and IZA \\ Christopher J. Ruhm \\ University of Virginia \\ and IZA
}
May 2011
IZA
P.O. Box 7240
53072 Bonn
Germany

Discussion Paper No. 5728

Phone: +49-228-3894-0

Fax: +49-228-3894-180

E-mail: iza@iza.org

\begin{abstract}
Any opinions expressed here are those of the author(s) and not those of IZA. Research published in this series may include views on policy, but the institute itself takes no institutional policy positions.

The Institute for the Study of Labor (IZA) in Bonn is a local and virtual international research center and a place of communication between science, politics and business. IZA is an independent nonprofit organization supported by Deutsche Post Foundation. The center is associated with the University of Bonn and offers a stimulating research environment through its international network, workshops and conferences, data service, project support, research visits and doctoral program. IZA engages in (i) original and internationally competitive research in all fields of labor economics, (ii) development of policy concepts, and (iii) dissemination of research results and concepts to the interested public.
\end{abstract}

IZA Discussion Papers often represent preliminary work and are circulated to encourage discussion. Citation of such a paper should account for its provisional character. A revised version may be available directly from the author. 


\section{ABSTRACT}

\section{The Economics of Risky Health Behaviors ${ }^{1}$}

Risky health behaviors such as smoking, drinking alcohol, drug use, unprotected sex, and poor diets and sedentary lifestyles (leading to obesity) are a major source of preventable deaths. This chapter overviews the theoretical frameworks for, and empirical evidence on, the economics of risky health behaviors. It describes traditional economic approaches emphasizing utility maximization that, under certain assumptions, result in Pareto-optimal outcomes and a limited role for policy interventions. It also details nontraditional models (e.g. involving hyperbolic time discounting or bounded rationality) that even without market imperfections can result in suboptimal outcomes for which government intervention has greater potential to increase social welfare. The chapter summarizes the literature on the consequences of risky health behaviors for economic outcomes such as medical care costs, educational attainment, employment, wages, and crime. It also reviews the research on policies and strategies with the potential to modify risky health behaviors, such as taxes or subsidies, cash incentives, restrictions on purchase and use, providing information and restricting advertising. The chapter concludes with suggestions for future research.

JEL Classification: I1, I20, I180, D01, H2, D1, D6, D03, D87, D83, J1, Q18, I24

Keywords: health behaviors, alcohol, tobacco, smoking, drugs, obesity, diet, food, physical activity, public health, public policy, taxation, subsidies, addiction, externalities, advertising, information, behavioral economics, neuroeconomics, human capital, education, prices, sex, income, time preference, peers, bounded rationality, medical costs, employment, wages, crime, hyperbolic discounting

Corresponding author:

John Cawley

Department of Policy Analysis and Management

Cornell University

124 MVR Hall

Ithaca NY 14853

USA

E-mail: JHC38@cornell.edu

\footnotetext{
${ }^{1}$ This is a paper in progress that will be published as a chapter in the Handbook of Health Economics, volume 2, by Elsevier. We thank the editors of the Handbook, Pedro Pita Barros, Tom McGuire, and Mark Pauly, for their useful feedback and helpful guidance. We also thank the other authors in the volume for their feedback and comments at the Authors' Conference, and we are grateful to Abigail Friedman for transcribing the comments at that conference.
} 


\section{Introduction}

Health has many determinants, including market goods and services such as medical care, investments of time, and environmental conditions such as air pollution, sanitation and water purity. However, in industrialized countries where morbidity and mortality are primarily related to chronic rather than infectious diseases, health behaviors are particularly important. Such health behaviors are the subject of this chapter and can be broadly construed as any action, or deliberate inaction, by an individual that affects his or her own health or the health of others. This chapter focuses on the specific behaviors - like smoking, drinking, diet and physical activity - that have strong direct effects on own health. The empirical evidence cited in this chapter is primarily drawn from high-income countries, particularly the United States, so the analysis is particularly relevant for industrialized nations. Previous Handbook chapters have provided detailed discussions of individual health habits such as smoking (Chaloupka and Warner, 2000) alcohol consumption (Cook and Moore, 2000) and prevention (Kenkel, 2000). For the most part, we neither repeat nor update those discussions. ${ }^{2}$ Instead, we provide a broad overview of theoretical frameworks and empirical evidence on the economics of health behaviors. In doing so, we examine traditional economics approaches emphasizing utilitymaximization that, under certain assumptions (e.g. perfect information and no externalities) result in Pareto-optimal outcomes and at most a limited role for policy interventions. We also describe a variety of factors (e.g. market imperfections and hyperbolic time discounting) that can result in Pareto suboptimal outcomes in which government intervention has the potential to increase social welfare.

\section{a. The Importance of Health Behaviors}

\footnotetext{
${ }^{2}$ For more recent, but also generally more selective, reviews of the literature on smoking, alcohol consumption and obesity, see: Gallet and List (2003); Wagenaar et al., (2009); Cook and Moore (2002) and Cawley (2010).
} 
An influential study by Mcginnis \& Foege (1993) estimated that approximately half of the 2.1 million deaths occurring in the United States in 1990 resulted from external modifiable risk factors. Their findings, summarized in the first column of Table 3.1.1, suggest that health behaviors play a major role. Tobacco use was responsible for almost a fifth of mortality in that year and the combined influence of smoking, diet, physical activity and alcohol consumption accounted for 38 percent of deaths. Unsafe sexual behaviors, driving and illicit drug use accounted for another 3 percent of deaths. An update to this study (Mokdad et al., 2004, 2005), shown in the second column of Table 3.1.1, indicated that the situation was relatively similar in 2000, when 36 percent of deaths were related to smoking, diet, physical activity, and alcohol consumption and an additional 3 percent of deaths were attributable to unsafe sex, driving, or drug use.

\section{INSERT TABLE 3.1.1 HERE}

The results of more recent research, examining a different set of risk factors for U.S. mortality in the 2005, are shown in Table 3.1.2 (Danaei et al. 2009). Whereas McGinnis and Foege (1993) and Mokdad et al. $(2004,2005)$ examine the composite risk factor of poor diet and physical inactivity, Danaei et al. (2009) separately break out the effects of: high BMI (to which they attribute 216,000 annual deaths), physical inactivity (191,000 deaths), high blood glucose (190,000 deaths), high LDL cholesterol (113,000 deaths), high dietary salt (102,000 deaths), low dietary omega-3 fatty acids (84,000 deaths), high dietary trans fatty acids (82,000 deaths), low intake of fruits and vegetables (58,000 deaths), and low dietary polyunsaturated fatty acids $(15,000)$.

INSERT TABLE 3.1.2 HERE 
All of these estimates should be interpreted with considerable caution because the sources of most deaths are multifactorial, making it quite difficult to ascertain the independent effect of specific determinants, and because of the difficulty of fully adjusted for potential confounding variables. $^{3}$ This uncertainty notwithstanding, modifiable behaviors represent an important determinant of premature death. In addition, mortality is only part of the negative consequences of poor health habits; morbidity must also be considered. For example, obesity is associated with high rates of arthritis, which is chronic and disabling but rarely deadly, and Type II diabetes, which can lead to medical complications such as blindness and amputation of toes or feet (Dixon, 2010). Smoking is similarly linked to a myriad of quality-of-life reducing health problems such as lung cancer, emphysema, and chronic obstructive pulmonary disease (US DHHS, 1990).

The World Health Organization (WHO) has recently examined how modifiable risk factors are related to both mortality and morbidity, as measured by disability-adjusted life years (DALYs) (World Health Organization, 2009). The results for high income countries (those with 2004 per capita incomes in excess of $\$ 10,065$ ), which are summarized in Table 3.1.3, differ from those presented in Tables 3.1.1 and 3.1.2 in that there is no attempt to identify a single (primary) cause of death or disability, nor to account for interactions between them (e.g. smoking may be a cause of hypertension). As a result, many of the risk factors may reflect a combination of health behaviors and medical treatments.

\section{INSERT TABLE 3.1.3 HERE}

\footnotetext{
${ }^{3}$ For example, Flegal et al. (2005) calculate that the number of excess deaths associated with clinical weight classification (relative to the normal weight category of $18.5<=$ BMI $<25$ ) was 112,000 for obesity (BMI $>=30$ ), negative 86,000 for overweight $(25<=\mathrm{BMI}<30)$, and 34,000 for underweight $(\mathrm{BMI}<=18)$, which is difficult to reconcile with the Mokdad et al. (2005) estimate that 365,000 deaths are due to poor diet and physical inactivity.
} 
The estimates from the WHO, shown in Table 3.1.3, rate smoking as the most damaging health behavior, responsible for 18 percent of deaths and 11\% of DALYs. Excess body weight is third on the list, responsible for 8 percent of deaths and 7 percent of DALYs, and physical inactivity is fourth, responsible for 8 percent of deaths and 4 percent of DALYs. The second, fifth and sixth ranked risk factors - high blood pressure, blood glucose, and cholesterol - are all affected by health behaviors such as smoking, physical inactivity and diet. Indeed, only two of the risk factors listed - outdoor air pollution and occupational risks - are unrelated to individual health behaviors.

The WHO analysis underscores the importance of individual health behaviors in modern industrialized economies. This is in contrast to poorer nations, where infectious diseases and environmental risks play a greater role. For example, in countries with 2004 per capita incomes of $\$ 825$ or less, the top ten risks of death included child underweight, unsafe water/sanitation/hygiene, and indoor smoke from solid fuels (ranked first, fourth and sixth), all of which are a direct consequence of poverty (poverty could, in turn, affect behaviors) . Low incomes are also important for suboptimal breastfeeding (ranked ninth) and limiting the availability of medical treatments that might offset the consequences of unsafe sex, which is the third ranked mortality risk. Tobacco use and physical inactivity play a smaller role in poorer countries - each being involved in around 4 percent of deaths and ranked as the seventh and eighth risk factors. ${ }^{4}$

\section{b. Trends in Health Behaviors}

\footnotetext{
${ }^{4}$ The risks associated with DALYs appear to be even more directly related to poverty. Child underweight, unsafe water/sanitation/hygiene, unsafe sex, suboptimal breastfeeding and indoor smoke from solid fuels are the top five risks, with vitamin A and zinc deficiencies ranking sixth and tenth. Rounding out the list are high blood pressure, alcohol use and high blood glucose (ranked seventh through ninth). Smoking, overweight/obesity, physical inactivity and illicit drug use are notably absent from this list.
} 
Figures 3.1.1 and 3.1.2 depict trends in a variety of health behaviors in the United States, based on information from a variety of sources (detailed in the figure notes). ${ }^{5}$ Figure 3.1.1 illustrates alcohol consumption per capita, the prevalence of current smoking among adults, illicit drug use during the past year by high school seniors, daily calorie intake (separately for males and females), and obesity prevalence. ${ }^{6}$ The results are mixed, with a trend toward healthier behaviors on some dimensions but not others. Most importantly, smoking prevalence fell by almost half among adults between 1974 and 2007 (from 37 to 20 percent), while obesity more than doubled (rising from 15 percent in the early 1970s to 34 percent in 2003-2006). The increase in obesity was fueled by an increase in average daily calorie consumption (12\% rise for males, and 23\% rise for females). Alcohol consumption per capita declined 20 percent between 1974 and 1997 but has increased modestly (by around 8 percent) since then. The effects of this change are ambiguous because light drinking may yield some health benefits (Gaziano, et al., 1993; Thun, et al., 1997). However, during the 1997-2007 period there was either no change or an increase in binge and heavy drinking (National Center for Health Statistics, 2010), which are likely to negatively affect health. ${ }^{7}$ Finally, illicit drug use (among high school seniors) shows no clear time trend: it fell sharply from a peak of 54 percent in 1979 to a trough of 27 percent in 1992, before rising rapidly to 42 percent in 1997 after which it declined modestly. ${ }^{8}$

\section{INSERT FIGURES 3.1.1 AND 3.1.2 HERE}

\footnotetext{
${ }^{5}$ Some databases provide information on health behaviors for a broader set of countries (although completeness and comparability of the data sometimes presents challenges. For instance, the OECD Health Data (www.oecd.org/health/healthdata) indicates food, alcohol and tobacco consumption, and rates of overweight and obesity, for most OECD countries. Data on these behaviors, as well as on physical activity, oral health and health risks such as blood pressure, cholesterol and diabetes can be obtained from the World Health Organization's, WHO Global Infobase (https://apps.who.int/infobase/).

${ }^{6}$ Data are unavailable in some years for many of these outcomes, with linear interpolation used to impute these missing values. Unless otherwise noted, obesity is defined throughout as a body mass index (BMI, calculated as weight in kilograms divided by height in meters squared) of 30 or higher.

${ }^{7}$ There is no evidence of reductions in heavy drinking (more than 14 drinks per week for males and 7 drinks per week for females) or binge drinking (5 or more drinks on a single occasion).

${ }^{8}$ Illicit drugs include: marijuana, LSD, other hallucinogens, cocaine, heroin, other narcotics, amphetamines, barbiturates, or tranquilizers not under a doctor's orders.
} 
Figure 3.1.2 depicts trends in health behaviors such as physical inactivity (no leisure-time physical activity during the last month), medical screening tests (mammograms and colorectal endoscopy), vaccinations (flu shots) and breastfeeding. Obviously, these represent only a subset of possible health behaviors that could be considered and their inclusion is illustrative rather than exhaustive. Consistent data on these behaviors is available for a shorter period of time (with the exception of breastfeeding) than those presented in the previous figure; however, they tend to suggest that healthy behaviors are becoming increasingly common over time. Thus, mammography and colonoscopies have both become more widely used over time, as has influenza vaccination and the fraction of infants that are breastfed. ${ }^{9}$ There also appears to have been an increase in physical activity, although most adults do not meet recommended levels (Carlson et al., 2008).

Overall, changes in health behaviors since the 1970s (particularly the rapid decline in smoking) have mostly operated in the direction of improving overall health (Cutler et al., 2009); however, many of these beneficial trends ended or slowed by the early or mid-1990s. Moreover, the main exception to this pattern - the rapid and continuing growth in obesity - has important negative consequences. As always, it is difficult to extrapolate from the past to predict the future. Cutler et al. (2009) suggest that mortality risks will increase during the first two decades of the $21^{\text {st }}$ century due to increases in obesity, ${ }^{10}$ and Olshansky et al. (2005) raise the possibility that its rise may lead to reductions in life expectancy. ${ }^{11}$

\section{c. Differences in Health Behaviors Across Population Subgroups}

\footnotetext{
${ }^{9}$ The results for breastfeeding are part of a longer-term secular increase. For instance, 30 percent of infants were breastfed (for at least some period of time) in 1974 as compared to 53 percent in 1990 and 77 percent in 2006.

10 Their estimates of mortality risk are not strictly limited to changes in health behaviors. In particular, they allow for direct effects of education and of blood pressure and cholesterol; the former influences health behaviors, while the latter are affected by them.

${ }^{11}$ However, Flegal et al. (2007) find that most types of mortality risk from obesity have been falling over time.
} 
Health-related behaviors differ, sometimes substantially, across population subgroups. Table 3.1.4 summarizes disparities in health behaviors like smoking, obesity, drinking, physical inactivity, two types of medical screening testing (mammograms and colorectal exams), sexually transmitted disease and the use of sun protection. The estimates are based on 2008 data from the National Health Interview Survey (NHIS). ${ }^{12}$ The subsamples examined are stratified by sex, race/ethnicity, age, education and annual family income; within those categories the averages and prevalences reported are unconditional. Because many of these factors may be correlated (i.e. better educated persons tend to be in families with higher incomes), the disparities observed should not be interpreted as causal. Table 3.1.5 displays probit estimates of the corresponding predicted subgroup differences after including controls for demographic characteristics.

\section{INSERT TABLES 3.1.4 and 3.1.5 HERE}

The two tables reveal fairly similar patterns. Females are more likely than males to engage in certain healthy behaviors (they are less likely to smoke or binge drink, and are more likely to use sunscreen) but are less likely than males to engage in other healthy behaviors (they are less likely to engage in physical activity and are more likely to have sexually transmitted diseases (STDs), although the STD disparity may be due biological differences in susceptibility rather than differences in behaviors).

Blacks and Hispanics are less likely than non-Hispanic whites to smoke or drink heavily, but are more likely to be obese and physically inactive. Minorities less commonly receive colorectal screening and Hispanic women are less likely to obtain mammograms but most of these differences are associated with correlated factors (like education and income) rather than race/ethnicity itself. Smoking and problem drinking tend to decrease with age, while obesity, physical inactivity and medical screening tend to rise.

\footnotetext{
${ }^{12}$ See http://www.cdc.gov/nchs/nhis.htm for information on the NHIS.
} 
By far the strongest results are that higher socioeconomic status (SES), as proxied by educational attainment or family income, is generally correlated with healthier behaviors. For example, compared to high school dropouts, college graduates were 13.9 percentage points less likely to smoke, 8.7 percentage points less likely to be obese, 0.9 percentage points less likely to drink heavily, and 22.3 percentage points less likely to be physically inactive. In addition, they are 12.6 percentage points more likely to receive mammograms, 15.3 percentage points more likely to receive colorectal screening and 16.0 percentage points more likely to use sunscreen when outside on warm sunny days. The only exception is that the highly educated were 2 percentage points more likely to have had an STD during the last 5 years. Income also appears to be associated with healthy behaviors, independent of education. Compared to persons with family incomes below $\$ 35,000$, those with family incomes of at least $\$ 75,000$ had relatively low rates of smoking, obesity, physical inactivity and STDs; they also have a high prevalence of medical screening and sunscreen use. The exception to this pattern is that high family income is associated with modestly greater rates of binge drinking.

Previous evidence of healthier behaviors by more advantaged individuals was obtained in the two influential "Whitehall studies” of British civil servants (Marmot et al., 1978, 1991), which documented a strong positive relationship between occupational status, healthy behaviors and life expectancy. ${ }^{13}$ In these, and in many subsequent examinations, drinking is an exception, as it was in the NHIS data discussed above. For example, Adler et al. (1994) provide evidence of a negative association between SES (usually proxied by income or education) and smoking or physical inactivity but a positive correlation of SES with alcohol consumption. Cutler \& LlerasMuney (2010) show that education is positively associated with healthier behavior as regards to smoking, diet and obesity, health knowledge, household safety, medical testing, screening and

\footnotetext{
${ }^{13}$ See Marmot and Wilkinson (2006) for an in-depth discussion of these issues.
} 
vaccinations and the control of high blood pressure and diabetes. Conversely, the highly educated were more likely to have ever used marijuana (but had smoked it less frequently within the last year) and had more often engaged in light (but not heavy) drinking.

Differences in health behaviors are one possible explanation for why socioeconomic status is positively related to health status and life expectancy. Interestingly, the original Whitehall studies, and much research that has followed (e.g. Lynch et al., 1996; Lantz et al., 2001), suggests that behaviors explain only a small fraction of the better health and longer life expectancy experienced by high individuals with high socioeconomic status. However, using data from the British Health and Lifestyle Survey, Contoyannis \& Jones (2004) and Balia \& Jones (2008) potentially resolve this contradiction, showing that accounting for endogeneity in behavioral choices increases the estimated effect of behaviors on health outcomes and reduces the size of the residual SES effects. This occurs because persons with worse latent health tend to adopt healthier behaviors (e.g. someone who is diagnosed with cancer may quit smoking), introducing a negative bias into the predicted effects of health-enhancing behaviors.

Over time, SES-related gradients have widened for most, but not all, health behaviors. Probably most dramatic is the change for tobacco use, where a large gap has emerged over the last four decades because of larger reductions in smoking for more advantaged adults. Kanjilal et al. (2006) present evidence from the National Health and Nutrition Examination Surveys (NHANES) showing that the gap in current smoking between persons with more versus less than a high school education was 11.6 percentage points (33.5\% vs. 45.1\%) in 1971-74 but had almost doubled to 21.5 points (17.1\% vs. 38.6\%) in 1999-2002; during the same period the smoking differential between the highest and lowest poverty-income-ratio (PIR) quartiles rose from 10.5 (33.5\% vs. $44.0 \%$ ) to 23.5 (13.9\% vs. 37.4\%) percentage points. Kenkel (2007) finds 
that the gap in smoking rates between those with and without a college degree grew from 2 percentage points in 1954 to 15 points in 1999. A substantial portion of the recent differential occurs because the highly educated tobacco users are much more likely than their less educated counterparts to quit smoking. For instance, in 2008, the quit ratio - defined as the percentage of persons who had ever smoked (more than 100 cigarettes) but who were not current tobacco users - was $45.7 \%$ for adults (aged 25 and over) without a high school diploma, compared to $80.7 \%$ of those with a graduate degree (Dube et al., 2009).

In contrast, most of the available evidence suggests that SES-related differentials in obesity have narrowed over time, as body weight has increased for all groups but at a somewhat faster rate for those with high education or income. Zhang \& Wang (2004) find that between 1971-74 and 1999-2000 the prevalence of obesity rose 16.2 percentage points (from $7.4 \%$ to 23.6\%) for college educated men (aged 20 to 60 ) and 14.7 percentage points (from $12.0 \%$ to 26.7\%) for those with less than a high school education. Over the same period the education gradient narrowed even more for women; the prevalence of obesity increased 22.6 percentage points (from $7.3 \%$ to $29.9 \%$ ) for college educated women versus 12.9 percentage points (from $24.9 \%$ to $37.8 \%$ ) for women with less than a high school education. The results of Chang \& Lauderdale (2005) are also suggestive of a weakening in the negative relationship between income and obesity between 1971-74 and 1999-2002, to the extent that income and obesity are now positively correlated for non-Hispanic Black males. Among low SES individuals, the prevalence of obesity was already quite high in the early 1970s and so has grown relatively little over time, raising the possibility that differentials in severe obesity might have continued to widen over time. However, Cutler, et al. (2010) find that the combined prevalence of Class 2 obesity (BMI between 35.0 and 39.9) and Class 3 obesity (BMI of 40 or higher) rose between 
1971-74 and 1999-2004 by the same amount for adults with and without college educations: by 8 percentage points for males and 11 percentage points for females in both education groups.

There is weaker evidence on the behaviors that determine body weight: diet and physical activity. Popkin et al. (1996) find improvements in dietary quality between 1965 and 1989-91, with larger gains for highly educated persons (who had worse diets at the beginning of the period but similar quality diets at the end of it). Casagrande et al. (2007) find that the consumption of fruits and vegetables changed little between 1988-94 and 1999-2002, with possibly higher levels and slight increases observed for high SES individuals. Conversely, Kant \& Graubard (2007) show that income and education-related differentials in the consumption of healthy foods declined between 1971-75 and 1999-2002, largely due to a decrease among advantaged individuals. All of these findings should be considered preliminary and are not informative about net energy intake, which is of primary interest when one is concerned about obesity.

There is even less evidence on whether and how energy expenditure has changed over time across groups. Simpson et al. (2003) find that the prevalence of walking rose between 1987 and 2000 but without clear differences across education. On the other hand, Brownson et al. (2005) document modest increases in the probability of achieving recommended levels of physical activity for persons with 16 or more years of education but decreases for those with fewer than 12 years of schooling. However, they emphasize that this includes only leisure-time physical activities, and so does not provide information on other sources of energy expenditure (e.g. the strenuousness of work), which has declined over time, quite possibly differentially across groups. $^{14}$

\footnotetext{
${ }^{14}$ There have also been important changes in a variety of medical conditions - like hypertension, high blood pressure, and diabetes mellitus - that are determined by an interaction of health behaviors and medical care. Better control of blood pressure and cholesterol, particularly when combined with reductions in smoking, represent significant improvements in cardiovascular risk factors. These have declined over the last three decades of the $20^{\text {th }}$
} 


\section{d. Health Economics Research on Health Behaviors}

The introduction to Volume 1A of the Handbook of Health Economics (Culyer and

Newhouse, 2000) includes an organizational chart of the field of health economics. Six of the eight categories of research relate to the health care sector and there is no explicit category for economics research on health behaviours; this is presumably included under the vague residual grouping of “what influences health, other than health care?” (see Cawley and Kenkel, 2008).

However, 50 percent of U.S. health economists surveyed in 2005 reported studying "the behavior of individuals," which makes this as popular as any sub-specialty of health economics (Morrisey and Cawley, 2008), and economics research on health behaviors has increased considerably in the past three decades. ${ }^{15}$ Figure 3.1.3 depicts the trend in the number of economics publications concerning various health behaviors identified in EconLit, the database of journal articles, dissertations, and working papers in economics. Between 1980 and 2009, the annual number of economics publications on obesity increased from 0 to 135, the number on alcohol rose from 2 to 99, those on tobacco or smoking grew from 31 to 410, and the number on health behaviors generally increased from 2 to 292. ${ }^{16}$ These trends are likely due to many factors, including greater awareness of the externalities associated with unhealthy behaviors (for instance, Manning et al.'s (1991) study on the external costs of smoking, heavy drinking, and sedentary life-styles), growth in the availability of large secondary datasets allowing researchers to track and model unhealthy behaviors (e.g. the Behavioral Risk Factor Surveillance System (BRFSS) began in

century for virtually all groups but without clear differences across education or income categories (Kanjilal, et al., 2006; Cutler, et al., 2010). Conversely, diabetes diagnoses have increased dramatically over time, particularly for those with less education or low incomes (Kanjilal, et al., 2006).

${ }^{15}$ While it seems likely that most of the health economists stating that they research "the behavior of individuals" study health behaviors, it is possible that some are referring to non-health behaviors.

${ }^{16}$ The annual number of publications in health care economics also rose substantially, but less than the increase in health behaviors research. For example, the annual number of publications in EconLit with the keyword "hospital" rose from 30 in 1980 to 235 in 2009, and the number with the keyword "medical care" rose from 5 in 1980 to 61 in 2009. 
1984 and the Youth Risk Behavior Surveillance System (YRBSS) started in 1991), and rising interest in government action to internalize the negative externalities associated with some health behaviors (e.g. the Federal tax increases on tobacco enacted during the 1990s).

\section{INSERT FIGURE 3.1.3 HERE}

The importance of health behaviors in explaining morbidity and mortality in economically developed countries, increasing richness of data available to study these behaviors, and the policy relevance of the related research questions, makes us optimistic about the future of research on health behaviors. We hope this chapter will be useful to the researchers contributing to that future literature and of broader interest to students and policy-makers.

We have chosen not to divide this chapter into sections for smoking, alcohol consumption, drug use, and obesity; instead, the chapter is organized by the underlying economic concepts that relate to all behaviors. It is our hope that this organization will enable researchers to see common patterns and important differences across the various health behaviors, and bring researchers of specific health behaviors out of their separate silos to learn from the synergistic research on other health behaviors.

Interested readers are also referred to the previous Handbook chapters on smoking (Chaloupka and Warner, 2000) alcohol consumption (Cook and Moore, 2000) and prevention (Kenkel, 2000), the chapter on health behaviors among young people in the Elgar Companion to Health Economics (Kenkel, 2006), and that on health behaviors and addictions in the Oxford Handbook of Health Economics (Kenkel and Sindelar, forthcoming).

\section{The Traditional Economic Approach to Studying Health Behaviors}

\section{a. Model of Health Capital}


The foundation for much economics research on health behaviors is Michael Grossman's model of health capital (Grossman 1972, 2000), which has been detailed in a previous volume in this series (Grossman 2000). ${ }^{17}$ Basic aspects of the model are that people receive an endowment of health capital at birth, which depreciates with age but can be raised through investments; death occurs when the health stock falls below a minimum level. Health has both consumption and investment aspects, as it enters the utility function directly and determines the amount of healthy time available for market and nonmarket activities. People produce health by combining market goods and services with time, consistent with Becker's model of household production (Becker 1976). For example, an individual might produce health by buying a treadmill and running shoes and spending time running on the treadmill.

Individuals allocate time and money to maximize the present discounted value of lifetime utility. Indirectly, length of life is a choice in the original model which contains no uncertainty. Specifically, the timing of death results from conscious decisions regarding health investments made with full knowledge of their implications for longevity. ${ }^{18}$ Assuming that health has only investment aspects (i.e. it does not enter the utility function directly and is only valuable for producing healthy days), optimal health capital is characterized by an equality of the supply of health capital (i.e. the opportunity cost of health capital) and the demand for health capital (i.e. the marginal monetary return on health investments).

Application of the health capital model for health behaviors such as sleep and exercise is straightforward (see Kenkel, 2000): people invest in such behaviors until, at the margin, the return on investments in health equals the opportunity cost of health capital. However, the

\footnotetext{
${ }^{17}$ Related empirical work is often inspired more by the intuition of this model than strict adherence to its theoretical features.

${ }^{18}$ However, at high ages, the depreciation rate of health capital may become so large that the individual is unable to afford sufficient investment flows to stay alive.
} 
model also applies to unhealthy behaviors, which can be interpreted as negative investments in health. When the individual has solved the constrained maximization problem, the optimal participation in unhealthy behaviors will be characterized by an equality of the marginal costs of the unhealthy behavior (both the monetary cost of purchasing market goods like cigarettes and alcohol and the nonpecuniary cost of reduced health and shorter lifespan) and the marginal benefits (such as the instantaneous pleasure derived from consumption).

\section{b. Education and Health Behaviors}

The literature review by Grossman and Kaestner (1997) concluded that education is the most important correlate of good health for both individuals and groups; in particular, health is more strongly correlated with schooling than with occupation or income. In the model of health capital (Grossman, 1972), schooling may improve health by enhancing allocative efficiency (participation in healthier behaviors) or productive efficiency (obtaining more health from the same set of inputs). Cutler and Lleras-Muney (2010) provide an overview of the differences in health behavior by education, noting that in a parsimonious model estimated using the National Health Interview Survey data, an additional year of education is associated with a 3.0 percentage point lower probability of being a current smoker, 1.4 percentage point lower probability of being obese, a 1.8 percentage point lower probability of being a heavy drinker, and a 0.1 percentage point lower probability of using marijuana in the past month.

Economists have used a variety of identification strategies to measure the causal effect of education on health behaviors. Focusing on the studies that used instruments that are both powerful and plausibly exogenous, the results are mixed. Three studies find evidence of a causal effect of education on smoking. Currie and Moretti (2003) instrument for a woman's education using college openings in her county of residence and find that education reduces the probability 
of smoking. deWalque (2007) and Grimard and Parent (2007) exploit college attendance as a draft avoidance strategy during the Vietnam war and both find that college education reduces the probability of smoking by males.

Other studies are unable to reject the null hypothesis of no causal effect of education on health behaviors. Reinhold and Jurges (2009) examine the exogenous variation in education due to the abolition of school fees in Germany and conclude that there is no evidence that education causes reductions in smoking or obesity. Clark and Royer (2010) exploit two changes to British compulsory schooling laws that compelled a large percentage of the student population to stay in school longer. This exogenous variation in education had no detectable effect on smoking, drinking, diet, or exercise.

Other studies find mixed results. For example, Kenkel et al. (2006) exploit education policies (e.g. number of courses required to graduate from high school) as instruments and find that high school completion significantly reduces the probability of current smoking by adult men (but not women), but has no effect on overweight or obesity for either men or women.

\section{c. Habit and Addiction}

Marshall (1920) may have been the first work in which an economist addressed the phenomenon of habit or addiction; it contains the observation that “...the more good music a

man hears, the stronger his taste for it is likely to become...” (Marshall, 1920, p. 94; Stigler and Becker, 1977). In this sense, the individual's utility function includes not just the current consumption of music $C$ but also the stock of past consumption of music $S$.

In general, there are three characteristics of addiction. Reinforcement implies that the marginal utility of current consumption rises with the stock of past consumption $\left(U_{C S}>0\right)$. This 
is called adjacent complementarity because consumption of the good in adjacent time periods is complementary. ${ }^{19}$ Tolerance implies that the stock of past consumption lowers utility $\left(U_{S}<0\right)$. This assumes that the addiction is harmful; one can also have a beneficial addiction (like exercise) where the stock of past consumption raises utility $\left(U_{S}>0\right)$. Finally, withdrawal implies that there is a positive marginal utility of current consumption $\left(U_{C}>0\right){ }^{20}$

These characteristics of addiction are depicted in Figure 3.2.1, which is adapted from Rachlin (1997). The vertical axis is utility $(U)$, and the horizontal axis is stock of past consumption of the habitual or addictive good $(S)$. The lower line AD shows the utility associated with each possible level of the stock of past consumption, conditional on not currently consuming the addictive good. The upper line BC shows the utility associated with each possible level of the stock of past consumption, conditional on currently consuming the addictive product. The graph illustrates withdrawal, as at every stock of past consumption, consuming the addictive good provides higher utility than abstaining: $U_{C}>0$. The graph also illustrates tolerance. It depicts a harmful addiction because the stock of past consumption lowers utility: $U_{S}<0$. For example, the first time an individual consumes the addictive substance, he has a stock of past consumption of zero, so he is at point B. After the individual has been heavy user for sufficiently long to have the maximum stock of past consumption, when he consumes he is at point $\mathrm{C}$, which is not only far below the utility he enjoyed during his first use of the addictive substance, it is also below the utility A that he enjoyed when he was still abstinent. Finally, the graph illustrates reinforcement, as the instantaneous marginal utility derived from consuming the

\footnotetext{
${ }^{19}$ Ryder and Heal (1973) also describe the possibility of distant complementarity, which they illustrate with the following example: "a person with distant complementarity who expects to receive a heavy supper would tend to eat a substantial breakfast and a light lunch. A person with adjacent complementarity would tend to eat a light breakfast and a substantial lunch in the same circumstances” (p. 5).

${ }^{20}$ More complicated (but realistic) models of withdrawal include a kink in the marginal utility function at or near levels of recent previous use, as detailed below.
} 
addictive good is greater the higher the stock of past consumption: $U_{C S}>0$. This illustrates that quitting a habit is harder the higher the stock of past consumption.

\section{INSERT FIGURE 3.2.1 HERE}

Houthakker and Taylor (1970) make the important empirical distinction between habitual (or addictive) goods, for which the stock of past consumption positively affects current use, and durable goods, for which prior consumption is negatively correlated with current use. They note that a good can be durable in the short term and habitual in the long-term. For example, even someone who habitually overeats will, in the short term, experience satiation (resulting in a negative correlation in consumption in nearby time periods) but over the longer term, consumption will be positively correlated and thus food is habitual over longer periods of time.

\section{i. Theory of Rational Addiction (TORA)}

A landmark in the study of addiction is Becker and Murphy’s (1988) Theory of Rational Addiction (TORA). In this model, addiction is optimal in the sense that it involves forwardlooking utility maximization with stable preferences. ${ }^{21}$ Previously, models of habit formation or addiction assumed that consumers were naïve: they realized that current consumption of the addictive good depended on its past consumption, but did not take into account the impact of current consumption on future consumption (Pollak, 1975). ${ }^{22}$ An appealing aspect of the TORA is that it allows consumers to be sophisticated regarding the intertemporal dynamics of consumption and utility.

The TORA assumes that instantaneous utility depends on current consumption of the addictive good, the stock of past consumption of the addictive good, and current consumption of all other goods. Individuals allocate income to addictive goods and all other goods, taking into

\footnotetext{
${ }^{21}$ This does not mean that addicts will necessarily be glad to be addicted, a point to which we return below.

${ }^{22}$ For an example model of myopic habit formation, see Houthakker and Taylor (1970).
} 
account the future consequences of their actions, including tolerance and reinforcement. ${ }^{23}$ In the TORA, a person for whom a good is highly addictive (i.e., for whom the good has high adjacent complementarity) might knowingly consume enough to become an addict, because he calculates that by doing so he maximizes the present discounted value of utility. Becker and Murphy describe the model as applicable to a wide spectrum of behaviors, including harmful addictions such as smoking, drinking, gambling, using cocaine or heroin, and overeating, as well as beneficial addictions such as religiosity and jogging.

Formally, the consumer maximizes the present discounted value of lifetime utility by allocating her budget to consumption of an addictive good $C$ and all other (nonaddictive) goods $Y$. Current utility depends not just on instantaneous consumption of $C$ and $Y$ but also on the stock of past consumption of $C$, which is denoted $S$. (The stock of past consumption of all other (nonaddictive) goods does not enter the utility function.) The lifetime utility function is:

$$
U(0)=\int_{0}^{T} e^{-\sigma t} U[Y(t), C(t), S(t)] d t
$$

where $\sigma$ is a constant rate of time preference. Reinforcement implies that $U_{C S}>0$ : a higher level of the addictive stock raises the marginal utility of consuming the addictive good.

Tolerance implies that $U_{S}<0$ for harmful addictions and that $U_{S}>0$ for beneficial addictions.

The stock of past consumption $S$ changes over time according to:

$$
\dot{S}(t)=S(t)-S(t-1)=C(t)-\delta S(t)-h[D(t)]
$$

where $C$ is consumption of the addictive good in period $t, \delta$ is the exogenous depreciation rate in the addictive stock, $D(t)$ represents expenditures on endogenous depreciation or appreciation of the stock. Consumers face a lifetime budget constraint.

\footnotetext{
${ }^{23}$ A parsimonious rational addiction model expresses the adverse future consequences of substance use as a higher level of addictive stock, which causes disutility, but one could also incorporate into the model other adverse future consequences, such as probability of martial strife, criminal victimization, or arrest.
} 
The TORA yields several important implications regarding the responsiveness of consumption to price. First, in almost any model of addiction, consumption at a point in time is related not only to current prices but also to past prices, because the latter determine the current addictive stock. Second, in models where agents exhibit foresight (like TORA but not myopic addiction), current consumption is also related to anticipated future prices because future prices will affect desired future consumption, which is a complement with the future level of the addictive stock, which in turn is affected by current consumption. Third, a future price change will have a greater impact on current demand the sooner it is anticipated, because individuals will then react to it earlier. Fourth, permanent price changes affect demand more than temporary ones, because forward-looking persons anticipate and make decisions based on future dynamics in prices. Fifth, the price elasticity of demand for the addictive good will be greater in the long run than in the short run, and that difference will rise with the level of addictiveness.

Finally, an arguably counter-intuitive implication is that the more addictive the good, the greater the long-run price elasticity of demand (See equation 18 and the related discussion in Becker and Murphy, 1988). The first reason that higher price leads to a reduction in quantity demanded is the usual reason that applies to all goods: the law of demand states that when price rises, the quantity demanded falls. However, for addictive goods there is a second reason that higher price leads to a reduction in quantity demanded: adjacent complementarity. Specifically, a rise in price that is expected to persist implies less consumption in the future, so it becomes optimal to hold a lower quantity of addictive stock, which is achieved by reducing consumption today. The more addictive the good, the greater the adjacent complementarity, and the greater the extent to which current consumption falls in response to an expected reduction in future consumption. This prediction - that all else equal addiction implies a greater price elasticity - is 
in stark contrast to early models of addiction that hypothesized that addicts were irrational and therefore unresponsive to incentives (see Cawley, 2008).

\section{ii. Empirical Tests of Rational Addiction}

Most papers testing the TORA use the empirical model developed by Chaloupka (1992) that represents a simplified version of the Becker and Murphy (1988) framework. The utility function is assumed to be quadratic, which yields linear first-order conditions and, with additional assumptions, the demand function:

$$
\mathrm{C}_{\mathrm{t}}=\beta_{0}+\beta_{1} \mathrm{P}_{\mathrm{t}}+\beta_{2} \mathrm{P}_{\mathrm{t}-1}+\beta_{3} \mathrm{P}_{\mathrm{t}+1}+\beta_{4} \mathrm{C}_{\mathrm{t}-1}+\beta_{5} \mathrm{C}_{\mathrm{t}+1}+\varepsilon,
$$

where $\mathrm{P}$ are prices and $\mathrm{C}$ are consumption levels of the addictive good in different time periods. ${ }^{24}$ If omitted determinants of demand are autocorrelated then lags and leads of consumption will be correlated with the residual of current consumption, and OLS estimates of (1) will yield biased estimates of $\beta_{4}$ and $\beta_{5}$. A common strategy for dealing with this problem is to instrument for lagged and future consumption using further lags and leads of prices, under the assumption that any effect on current consumption of prices before $(\mathrm{t}-1)$ or after $(\mathrm{t}+1)$ must operate through their effects on consumption in ( $\mathrm{t}-1)$ or $(\mathrm{t}+1)$ (Becker, Grossman and Murphy, 1994; Chaloupka, 1991).

The signs of the coefficients in (1) are used to test for addiction and forward-looking behavior. Table 3.2.1 lists predictions about the signs of the key coefficients implied by alternative hypotheses. Regardless of whether the good is addictive, consumption is always negatively correlated with contemporaneous price, because of the law of demand. The key test for addiction is whether past consumption raises current consumption; this is informative about

\footnotetext{
${ }^{24} \mathrm{An}$ interesting feature of this regression model is that the estimated coefficients on past and future prices, and past and future consumption, can be used to calculate the rate of time discount $(\sigma)$ by noting that $\beta_{3}=\beta_{2} /(1+\sigma)$ and $\beta_{5}=$ $\beta_{4} /(1+\sigma)$.
} 
adjacent complementarity. The key test for whether addiction is farsighted is whether current and future consumption are positively correlated - a rational (forward-looking) addict considers future events when choosing current consumption.

\section{INSERT TABLE 3.2.1. HERE}

Table 3.2.1 also shows the possibly surprising prediction of the TORA that past and future prices are positively correlated with current consumption, after controlling for past and future consumption (see the discussion in Chaloupka (1992) concerning equations 2 and 3). ${ }^{25}$ Because the model already controls for past and future consumption, for past (or future) prices to be higher holding constant past (or future) consumption, some unobserved correlate of demand must have changed. It is assumed that the change in unobservables persists today, implying higher demand today. ${ }^{26}$

Empirical tests of rational addiction have been conducted for many goods including: tobacco (Becker, Grossman and Murphy, 1991; Chaloupka, 1992), alcohol (Waters and Sloan, 1995), cocaine (Grossman and Chaloupka, 1998), obesity (Cawley, 1999), and gambling (Mobilia, 1992). Comprehensive reviews are available in Grossman (1993), Chaloupka (1996), Chaloupka (2000), and Cook and Moore (2000).

There are several challenges beyond those already mentioned to estimating empirical models of rational addiction. First, data on consumption of addictive goods may contain significant reporting error. Individuals may fear prosecution if they report consumption of illegal

\footnotetext{
${ }^{25}$ Gruber and Koszegi (2001) point out that, in many other applications, a positive correlation between future prices and current consumption is interpreted as a failed specification test of the model, not as evidence of forward-looking behavior.

${ }^{26}$ In the Chaloupka (1992) model, the long-run price elasticity of demand, defined as movement from one steady state of addictive consumption to another, is: $\varepsilon=\left(\partial \mathrm{C}^{*} / \partial \mathrm{P}\right)\left(\mathrm{P} / \mathrm{C}^{*}\right)=\left(\beta_{1}+\beta_{2}+\beta_{3}\right) /\left(1-\beta_{4}-\beta_{5}\right) *(\mathrm{P} / \mathrm{C} *$ ).
} 
substances, and stigma may lead to underreporting their use, even if legal. ${ }^{27}$ Also, heavy consumers of some addictive products may be unable to accurately recall their consumption. Moreover, sales data provide a noisy measure of consumption because some purchases may be shared with others, stored until later, wasted, or transported across borders. In future research, biomarkers (e.g. levels of nicotine, alcohol, or drugs in the blood) could serve as more objective measures of consumption but, to provide statistical power, would need to be collected (ideally repeatedly) for large numbers of people. ${ }^{28}$

A second empirical challenge is that prices are generally measured with error. This especially true for illegal drugs, but it can be remarkably challenging to determine prices faced by consumers even for cigarettes or alcohol because there many varieties and brands (which may vary in quality), and because purchases may occur in a different state with a lower tax rate, on Native American reservations with no excise taxes, or over the internet. Even when prices are accurate, consumers act in based on anticipated future prices that the econometrician does not observe.

Finally, some papers estimate variants of the rational addiction model using aggregate data, but these are unconvincing as there is no reason to believe that adjacent complementarity at the individual level should be detectable in aggregate data (Ferguson, 2000). Auld and Grootendorst's (2004) falsification test finds evidence of rational addiction, using conventional estimation techniques, for annual national aggregate quantities of milk, eggs, and oranges for Canada between 1961 and 2000. Moreover, their estimates suggest that milk is more addictive than cigarettes. They show that rational addiction can generally not be distinguished from serial

\footnotetext{
${ }^{27}$ This may be especially true in certain subpopulations. For instance, pregnant women may be more likely to underreport smoking or drinking than the general population.

${ }^{28}$ In an early use of biomarkers to study health behaviors, Farrell and Fuchs (1982) used carbon monoxide in expired air samples and thiocyanate in blood samples to confirm the accuracy of self-reports of smoking in the Stanford Heart Disease Prevention Program data.
} 
correlation when one uses aggregate time-series data and that the discount rates implied in such situations are unreliable. $^{29}$

\section{iii. Less-Tested Implications of TORA}

Under the TORA, greater adjacent complementarity implies a higher likelihood of unstable steady states. Becker and Murphy (1988) observe that, for many addictive goods, the distribution of consumption is bimodal. For example, few people consume small quantities of crystal meth or crack cocaine year after year; people tend to quickly converge to either a steady state with high consumption (addiction) or one with zero consumption (abstinence). Becker and Murphy suggest that exogenous shocks such as job loss or divorce could nudge a person out of the abstinence steady state, resulting in a rapid transition to the addicted steady state.

Conversely, a shock such as a bad drug experience or "bottoming out" could lead an addict to quit “cold turkey” and eventually end up abstaining from use. ${ }^{30}$

In contrast to the large number of studies using empirical models of rational addiction to calculate price elasticities of demand, we know of no empirical work examining unstable steady states or the possibility that shocks drive a person from abstinence to addiction or vice versa. This may be due to a lack of large longitudinal datasets that include both accurate data on addictive consumption and credibly exogenous shocks for a substantial number of respondents. Moreover, the shocks leading to movement between steady states may be idiosyncratically person-specific and therefore difficult to identify in secondary data.

\footnotetext{
${ }^{29}$ As an alternative, Gruber and Koszegi (2001) examine responses to increases in future cigarette taxes that have been enacted but not yet implemented. They find that cigarette sales rise but consumption falls, suggesting that consumers are both stock-piling (to avoid the higher future prices) and reducing smoking (to reduce the future addictive stock), both consistent with forward-looking behavior.

${ }^{30}$ Becker and Murphy argue that, when addiction is strong, the only effective way to quit is to go "cold turkey" because any consumption is likely to lead the consumer back to the addicted steady state.
} 
Consumption of addictive goods is sometimes characterized by cyclicality; for example, binging and purging with food, repeatedly quitting drinking but then falling off the wagon, or cold turkey drug withdrawal followed by subsequent use. The original Becker and Murphy (1988) model, and the elaboration on it by Dockner and Feichtinger (1993), permit such cycles of addictive behavior by incorporating a second addictive stock into the utility function. Whereas the original addictive stock exhibits adjacent complementarity, the second stock exhibits adjacent substitutability (i.e. higher values of the stock lower the marginal utility of consumption). The first stock is assumed to have a high rate of depreciation (i.e. it is very sensitive to recent consumption) while the second stock depreciates more slowly (i.e. it is less sensitive to recent consumption). This difference between the stocks in depreciation can generate rational consumption cycles. Suppose, for example, that an individual who has previously always abstained from the addictive good (so that both stocks are zero) begins to use the substance. The first addictive stock, which has a high depreciation rate (making it sensitive to recent events) increases rapidly, and the adjacent complementarity of the stock promotes greater future consumption. However, as the individual continues to consume in period after period, the second addictive stock (that has a low depreciation rate) increases. Eventually the effect of the second stock (characterized by adjacent substitutability) overwhelms the effect of the first stock (characterized by adjacent complementarity), and consumption begins to fall. The stock with adjacent complementarity depreciates quickly, and that with adjacent substitutability more slowly, so consumption remains relatively low even as both stocks depreciate. At some point, both stocks have fallen back to zero and the cycle may begin anew.

We are unaware of studies that have empirically tested the two-stock model of rational addiction. One reason may be that it is hard to measure or even define the two stocks (and little 
effort has been made in the theoretical literature to do so). It might be possible to define the stock with adjacent complementarity as "addiction" and that with adjacent substitutability as "bad health" but there is no clear justification for doing so and these concepts are difficult to measure. Other models that emphasize adjustment costs of deviating from recent levels of consumption (e.g. Dragone, 2009) may prove easier to estimate and test.

\section{iv. Rational Addiction with Learning and Uncertainty}

A potential criticism of the TORA is that it predicts that addicts should be satisfied with their consumption patterns or, at least, view it as preferable to alternative states of the world in which they consume less of the addictive product and have a lower addictive stock. In an important extension, Orphanides and Zervos (1995) point out that this need not hold once the assumption of perfect foresight is relaxed. Specifically, inexperienced individuals are assumed to be uncertain about the harm of consuming the addictive good, with learning occurring over

time. Persons with high addictive tendencies or excessive initial optimism (about the probability of avoiding addiction) will most frequently end up being addicts and will regret their addiction once it occurs.

Orphanides and Zervos (1995) assume there are two types of individuals: “non-addicts”, for whom $\theta=0$, and "potential addicts", for whom $\theta=1$. Individuals do not initially know to which group they belong but at time $\mathrm{t}$ assign a subjective probability, $\mathrm{P}(\mathrm{t})$, to likelihood that they are non-addicts. Utility at time $\mathrm{t}$ is:

$$
\mathrm{U}(\mathrm{t})=\mathrm{U}(\mathrm{Y}(\mathrm{t}), \mathrm{C}(\mathrm{t}))+\theta \eta(\mathrm{t}) v(\mathrm{C}(\mathrm{t}), \mathrm{S}(\mathrm{t}))
$$

where $\mathrm{U}($.$) represents the immediate reward from consuming the non-addictive good (Y), and the$ addictive product (C), $v$ is the detrimental addictive side-effect of past consumption, $\mathrm{S}$ is the stock of addictive capital and $\eta$ is a dichotomous variable indicating whether a harmful addiction 
has occurred, with $\operatorname{Pr}(\eta=1)$ is increasing in S. Individuals maximize discounted expected lifetime utility:

$$
\max \mathrm{E}\left\{\sum_{t=0}^{\infty} \delta^{t}[\mathrm{U}(\mathrm{Y}(\mathrm{t}), \mathrm{C}(\mathrm{t}))+\theta \eta(\mathrm{t}) v(\mathrm{C}(\mathrm{t}), \mathrm{S}(\mathrm{t}))]\right\}
$$

subject to a budget constraint and the equation of motion of the stock of addictive capital. $\delta$ is the discount factor.

The key distinction between this and the standard TORA model is the uncertainty in the last term: individuals do not initially know their type $\theta$ or the exact point at which addiction occurs. The subjective probability of being a non-addict $(\theta=0)$ is initially set at $\mathrm{P}(0)$ and changes based on a Bayesian updating rule. If $\theta \eta(t)>0$ then the consumer updates his beliefs so $\mathrm{P}(\mathrm{t}+1)=0$. In other words, harm from consuming A reveals to the individual that he cannot be a non-addict. Conversely, if $\theta \eta(t)=0$, the consumer updates his beliefs so $\mathrm{P}(\mathrm{t}+1)>\mathrm{P}(\mathrm{t})$, because consuming the addictive good without harm makes it more likely that he is a non-addict.

As in the standard model of rational addiction, the particularly interesting cases involve multiple steady-states, and Orphanides and Zervos focus on the situation with two equilibria for potential addicts: one a low level of consumption capital, $\mathrm{S}_{1}$, where harm does not occur, and the other a high and harmful level of addictive consumption, $\mathrm{S}_{2}{ }^{31}$ The likelihood that potential addicts end up at the higher steady-state primarily depends on the baseline probability $\mathrm{P}(0)$, with individuals who are overoptimistic (i.e. have too large a value of $\mathrm{P}(0)$ ) at greatest risk of becoming addicted. By construction, all addicts regret their decision, ex post. ${ }^{32}$ The initial value of the crucial subjective probability, $\mathrm{P}(0)$, is determined outside the model but the potential

\footnotetext{
${ }^{31}$ Non-addicts consume an amount of A that would lead to a stock intermediate between $S_{1}$ and $S_{2}$ if they were addicts.

${ }^{32}$ Wang (2007) extends upon the framework of Orphanides and Zervos in three ways. His model is solved in continuous rather than discrete time. There is uncertainty about the ability to stop using the addictive product, as well as in the probability of becoming an addict - thus quitting behavior (including unsuccessful quit attempts) is the focus of this model. Finally, all individuals are potential addicts, but with initially unknown heterogeneity in the threshold for addiction.
} 
importance of peer influences, unwarranted optimism, and misinformation are emphasized. All of these determinants yield policy implications that are potentially quite different than those from the original TORA model (e.g. government policies might influence values of $\mathrm{P}(0)$ ) and none have received detailed empirical examinations.

\section{d. Price Elasticities of Health Behaviors}

There are enormous research literatures estimating the price elasticity of demand for habitual or addictive substances. There are comprehensive reviews of this literature available for tobacco products (Chaloupka and Warner, 2000; Gallet and List, 2003), alcohol (Cook, 2000; Wagenaar et al., 2009), and food (Andreyeva et al., 2010). Grossman (2005) summarizes the empirical evidence on the importance of price as a determinant of the demand for cigarettes, alcohol, and illicit drugs. In this section we will cite the consensus estimates reported in the reviews and meta-analyses, and single out for special mention some studies that utilize exceptionally rich data or especially insightful methods. However, we caution that these studies vary in terms of data utilized and models estimated, so for specifics readers should refer to the original studies as well as the comprehensive literature reviews cited above.

Early studies estimated price elasticities of demand using aggregate data on sales and state taxes, and as a result were limited by multicollinearity, the discrepancy between sales and consumption, smuggling and cross-border shopping, and an inability to estimate elasticities separately for important subgroups such as youths or to measure the price elasticity of initiation or cessation. In response to these limitations, and thanks to the arrival of richer data, more recent studies have used longitudinal individual-level data on consumption. However, challenges persist in accurately measuring price, which may vary even within small geographic areas, and at any location vary by brand and quantity purchased. Common data sources for prices are: for 
tobacco the Tax Burden on Tobacco (Orzechowski and Walker, 2009), for alcohol and food the American Chamber of Commerce Researchers Association (or ACCRA) Cost of Living Index, and for illicit drugs the System to Retrieve Information from Drug Evidence (STRIDE).

Cigarettes are the addictive substance for which the most price elasticities of demand have been estimated. The Handbook of Health Economics chapter on smoking concludes that the price elasticity estimates for overall cigarette demand mostly fall within the range of -0.3 to 0.5 (Chaloupka and Warner, 2000). More recently, Gallet and List (2003) located 523 published estimates of the price elasticity of demand for cigarettes, the mean of which is -0.48 , with a large standard deviation (0.43) and ranging from -3.12 to 1.41. They find that the median estimate of the price elasticity of demand for cigarettes is larger for the long run $(-0.44, \mathrm{~N}=155)$ than the short run $(-0.40, \mathrm{~N}=368)$, and larger for men $(-0.50, \mathrm{~N}=24)$ than women $(-0.34, \mathrm{~N}=15)$. Aggregation of the data matters; the 87 studies using individual-level data found a smaller median price elasticity (-0.39) than the 101 studies using data at the level of the state or province (-0.60). See Table 3.2.2. for the median price elasticities of demand for cigarettes for different samples. The meta-analysis by Gallet and List (2003) finds that cigarette price elasticities are not affected by many aspects of the empirical model (e.g. whether account for myopic addiction or estimate a double hurdle model, whether data are time series or cross-sectional, or whether data are from before or after the release of the 1964 Surgeon General's report on smoking); they conclude that estimation methods have little or no impact on the price elasticity of demand for cigarettes.

Some studies separately estimate the impact of price on smoking at the extensive margin. The consensus price elasticity of smoking participation is around -0.5 (Gilleskie and Strumpf, 2005; Grossman, 2005). Gilleskie and Strumpf (2005) show that higher cigarette prices lead to 
particularly large decreases in the probability of initiation by nonsmokers. Conversely, in their preferred estimates, DeCicca, Kenkel, and Mathios (2002) find no impact of cigarette taxes on smoking initiation by youths (both genders pooled) and, estimating models separately by gender, Cawley, Markowitz, and Tauras (2004) estimate that smoking initiation by boys, but not girls, is sensitive to cigarette price.

\section{INSERT TABLE 3.2.2. HERE}

The next largest relevant literature on price elasticities concerns food. Andreyeva et al. (2010) locate 160 studies that calculate the price elasticity of demand for major food categories. They find that the mean price elasticity of demand for food away from home is $-0.81(\mathrm{~N}=13)$, for soft drinks is $-0.79(\mathrm{~N}=14)$, for fats and oils is $-0.48(\mathrm{~N}=13)$ and for sweets and sugars is -0.34 ( $N=13)$. Consumption of high-nutrient, less energy dense foods is also sensitive to price; the mean price elasticity of demand for fruit is $-0.70(\mathrm{~N}=20)$ and that for vegetables is $-0.58(\mathrm{~N}=20)$. The few studies that calculated price elasticities of demand for food separately by income group found essentially no difference between the price sensitivity of low-income consumers and the population as a whole (Andreyeva et al., 2010). See Table 3.2.3 for mean price elasticities of demand for various food categories.

\section{INSERT TABLE 3.2.3. HERE}

There are also a large number of studies estimating the price elasticity of demand for alcohol. Wagenaar et al. (2009) located 112 such studies containing a total of 1,003 estimates of price elasticity. The simple means of these price elasticities are -0.51 for alcohol as a whole ( $\mathrm{N}=91),-0.46$ for beer $(\mathrm{N}=105),-0.69$ for wine $(\mathrm{N}=93)$, and -0.80 for spirits $(\mathrm{N}=103)$, and -0.28 for heavy drinking $(\mathrm{N}=10)$. They conclude that there is "overwhelming” evidence that higher 
prices decrease consumption of alcohol (Wagenaar et al., 2009, p. 187). See Table 3.2.4A for price elasticities of demand for alcohol for different types of alcohol.

\section{INSERT TABLE 3.2.4A HERE}

Gallet (2007) conducts a meta-analysis of 132 studies of the price elasticity of demand for alcohol. Across 1,172 published estimates, the median price elasticity is -0.535 . The median price elasticity of demand for alcohol is larger in the long run $(-0.816, N=148)$ than in the short run $(-0.518, N=1,024)$. The one study located by Gallet (2007) that estimated the price elasticity of demand for alcohol separately by gender found that price elasticity is larger for women (0.750) than men (-0.509). See Table 3.2.4B for price elasticities of demand for alcohol for different samples.

\section{INSERT TABLE 3.2.4B HERE}

Some studies estimate the price elasticity of demand for alcohol at the extensive margin. For example, Manning, Blumberg, and Moulton (1995) calculate that a 10 percent increase in the price of alcohol decreases by 5.5 percent the probability that an individual is a current drinker.

A much smaller literature examines the price elasticity of demand for illicit drugs, generally focusing on the extensive margin of use; this literature confirms that even drug use is sensitive to price. Pacula et al. (2001) find that a 10\% increase in the price of marijuana decreases its use at the extensive margin among high school seniors by 3\%. Even the use of hard drugs is price-sensitive. A permanent $10 \%$ increase in the price of cocaine is estimated to reduce the probability of its use by approximately $10 \%$ and to reduce by 3 to 4 percent the number of times cocaine users take the drug (Grossman and Chaloupka, 1998; Chaloupka, Grossman, and Tauras, 1999). The price elasticity of heroin participation is -0.89 , and is also similar across race 
and gender groups (Saffer and Chaloupka, 1999). See Table 3.2.5 for price elasticities of demand for various illicit drugs.

\section{INSERT TABLE 3.2.5. HERE}

Others have estimated price elasticities of demand for opium based on historic data from East Asia. Van Ours (1995) examines the opium market in the Dutch East Indies for 1923-1938 and estimates that the short-term and long-term price elasticity of demand were about -0.7 and 1.0. Liu et al. (1999) uses data from the opium market in Taiwan for 1914-1942 and calculate that the short and long-run elasticities of demand were -0.48 and -1.38 .

An important question is whether price sensitivity varies by intensity of use; i.e. when prices rise, is the reduction in consumption limited to casual users, or do heavy users decrease their consumption as well? The answer varies by substance. For alcohol, there is strong consistent evidence that the heaviest drinkers are the least sensitive to price. Manning et al. (1995) find that the price elasticity of demand for alcohol is U-shaped across drinking intensity; demand is relatively inelastic (-0.55) at the fifth percentile of drinkers, price elastic (-1.19) for the median drinker, and essentially zero at the 95th percentile. Likewise, Wagenaar et al. (2009), in their review of the literature, find a mean price elasticity of heavy drinking of- $0.28(\mathrm{~N}=10)$, which is only a third of the overall price elasticity of alcohol consumption of $-0.91(\mathrm{~N}=91)$. Similarly, Cook and Moore (2001) estimate that a one-dollar increase in the beer excise tax would reduce the prevalence of youth alcohol use by two percentage points, but would have no effect on binge consumption (Cook and Moore, 2001). In models estimated separately by gender, Markowitz and Grossman (2000) find that heavy drinking is elastic to the price of beer for women, but not men. 
In contrast to alcohol, when it comes to food the heaviest consumers may be the most price sensitive. Auld and Powell (2009) estimate quantile regressions, which indicate that food prices have small effects on most of the population but have larger effects on youths above the $80^{\text {th }}$ or so quantile of the distribution of BMI; e.g. the effect of fast food prices at the $90^{\text {th }}$ or $95^{\text {th }}$ quantile are three to five times higher than estimates for the entire population. Evidence is more mixed for smoking; price is a greater deterrent to heavy smoking (11+ cigarettes per day) than lighter smoking (6-10 cigarettes per day), but demand is relatively price-elastic at both amounts (Gilleskie and Strumpf, 2005).

Another important question is whether youths are more or less price sensitive than adults, and the results are mixed. Gallet and List (2003), in their review of the literature, find that price elasticities of demand for cigarettes are larger for teens $(-1.43, \mathrm{~N}=8)$ and young adults $(-0.76$, $\mathrm{N}=22)$ than for adults $(-0.32, \mathrm{~N}=17)$. However, more recent studies using richer data suggest that price has less impact on the smoking initiation of youths than that of adults (DeCicca, Kenkel, and Mathios, 2002; DeCicca et al., 2008a,b). When panel data are treated as repeated cross-sections, this research obtains estimates similar to those obtained in previous crosssectional studies; e.g. the estimated price elasticity of teen smoking participation is around -0.7. However, when he longitudinal nature of the data is exploited by examining the smoking initiation decisions of nonsmoking youths, cigarette taxes are found to have little impact on the probability of smoking initiation.

For alcohol, the literature review by Gallet (2007) found a mean price elasticity of demand that was lower for young adults $(-0.386, \mathrm{~N}=13)$ than for adults $(-0.386, \mathrm{~N}=22)$. Saffer and Chaloupka (1999) find that the price elasticity of demand for cocaine and heroin is similar across age groups (Saffer and Chaloupka, 1999). 
Another important question is whether the health behaviors of pregnant women are sensitive to price. Two studies (Colman, Grossman, and Joyce, 2003; Gruber and Koszegi, 2001) find that a $10 \%$ increase in cigarette prices is estimated to cause $10 \%$ of women to stop smoking during pregnancy. An important direction for future research is to better understand how price elasticities of demand vary across unhealthy behaviors, types of consumers (especially youths and pregnant women) and amounts of use.

\section{e. Income and Health Behaviors}

Income could either increase or decrease unhealthy behaviors. Income could lead to a rise in unhealthy behaviors if cigarettes, alcohol, drugs, and food are normal goods. However, good health and appearance may also be normal goods, leading one to invest more time and money the production of health as income rises (Philipson and Posner, 1999).

Hundreds of published studies have calculated the income elasticity of smoking and drinking. Gallet and List (2003) located 375 published estimates of the income elasticity of cigarette smoking, the mean of which is 0.42 , with a standard deviation equal to 0.49 and ranging from -0.80 to 3.03. They find that the median estimate of the income elasticity of demand for cigarettes is greater in the long run $(0.39, \mathrm{~N}=80)$ than in the short run $(-.28, \mathrm{~N}=295)$, and are greater for women $(1.23, \mathrm{~N}=8)$ than men $(0.27, \mathrm{~N}=11)$. Aggregation of the data matters; the 10 studies using individual data found a median income elasticity of 0.06 , whereas the 24 studies using data at the level of state or province found a median income elasticity of 0.30 . See Table 3.2.6 for estimates of the income elasticity of smoking for various samples.

INSERT TABLE 3.2.6. HERE 
Gallet (2007) documents 1,014 published estimates of the income elasticity of demand for alcohol, for which the median estimate is 0.69 . As found for the price and income elasticities of demand for smoking, the income elasticity of demand for alcohol is sensitive to the aggregation of the data, with studies based on individual data finding a smaller median elasticity than those based on data at the state or province level. See Table 3.2.7 for estimates of the income elasticity of alcohol consumption for various samples.

\section{INSERT TABLE 3.2.7. HERE}

In order to measure the causal effect of income on health behaviors, researchers have exploited a variety of natural experiments. A few papers have been able to use lottery winnings as an exogenous source of variation in income. Lindahl (2005) finds that higher lottery winnings reduce the probability of being overweight, but Apouey and Clark (2010) find that lottery winnings lead to an increase in smoking and social drinking. Other research uses variation in government income transfer policies as a source of exogenous variation. Cawley et al. (2010) find no detectable impact of Social Security income on weight or obesity, using as a natural experiment the Social Security benefits notch that endowed certain cohorts of retirees with higher benefits. Schmeiser (2009) exploits variation across states in the generosity of the Earned Income Tax Credit (EITC) and is unable to reject the null hypothesis of no effect of income on weight for men. His results for women indicate that an additional \$1000 per year is associated with a gain of between 0.84 and 1.80 pounds. In a randomly-assigned conditional cash transfer program in Mexico, a doubling of cash transfers to a household was associated with significantly higher BMI and prevalence of obesity among adults (Fernald, Gertler, and Hou, 2008) but significantly lower BMI for age and prevalence of overweight among children (Fernald, Gertler, Neufield, 2008). 


\section{f. The Role of Advertising}

Economists have long debated how advertising affects consumer welfare. One possibility is that it provides valuable information about product attributes, quality, price, and lowers search costs. Alternatively, advertising may change consumer preferences or differentiate product in superficial ways, allowing higher prices to be charged. Joseph Stiglitz states flatly, "Most advertising is not informative. The typical Marlboro ad, with a cowboy smoking a cigarette, or a Virginia Slims ad, or a Budweiser Beer ad conveys no credible information concerning the nature of the product being sold, the price at which the product is sold, or where the product may be obtained.” (Stiglitz, 1989, p. 842).

Advertising may allow oligopolists to differentiate their products, reduce cross-price elasticities of demand and thereby avoid price competition. This was the strategy of U.S. cigarette manufacturers in the early 1920s, when producers tacitly colluded to keep prices high, and competed only on the basis of advertising, creating substantial barriers to the entry of potential new rivals (Adams, 1952).

The net effect of advertising may partly depend on the characteristics of a good. Advertisements for "search goods" - whose qualities are well-known to consumers - may focus on price and availability, whereas those for "experience goods" - whose qualities can only be determined upon consumption - may include relatively little factual information on price or product characteristics (Carlton and Perloff, 2000). ${ }^{33}$ This may be even truer for credence goods, whose qualities may be difficult to evaluate, even after consumption. For example, a smoker may buy low-tar cigarettes under the assumption that such cigarettes are less harmful to the smoker's health, but whether that is true will not be clear even after the cigarettes are smoked.

\footnotetext{
${ }^{33}$ For example, antidepressants are experience goods because they have idiosyncratic effects (regarding efficacy and side effects) that are only revealed (to the patient and physician) after being used for an extended period of time.
} 
Deceptive advertising is particularly advantageous to firms selling experience or credence goods. ${ }^{34}$

Adding complexity is that advertising may be a complement to consumption of the advertised good, enhancing welfare even if it does not provide specific useful information about product characteristics or price (Becker and Murphy, 2003). However, Stiglitz (1989) expresses reservations about this possibility.

It is unclear to what extent unhealthy behaviors involve search, experience, or credence goods. Brands of cigarettes, alcohol, and food that one has not yet tried are experience goods. For many such goods, each unit of a specific brand is homogenous, so after having tried the product once it becomes a search good. However, some product attributes may not be known even after consumption (e.g. the long-run health consequences of use), so there are certain ways in which these are credence goods.

Another ambiguity is whether advertising is cooperative (expanding the market by convincing new people to begin consuming the good) or competitive (increasing the advertised brand's share of a fixed market by stealing users from rival firms) or both. Limited empirical suggests that advertising of soft drinks is competitive (Gasmi, Laffont, and Vuong, 1992) while cigarette advertising is cooperative (Roberts and Samuelson, 1988).

Researchers examining how advertising influences unhealthy behaviors face several challenges, beyond those already mentioned. It is hard to measure an individual's exposure to advertising, or to find data that includes both exposure to advertising and consumption of the advertised good. Perhaps most importantly, it is difficult to exploit exogenous variations in advertising in order to identify its impact on consumption. As pointed out by Avery et al. (2007,

\footnotetext{
${ }^{34}$ For example, all of the 58 advertisements (run during the first half of 1965) found to be deceptive by the Federal Trade Commission concerned experience, rather than search, qualities (Nelson 1974).
} 
p. 449): "The relationship between advertising and consumption is literally a textbook example of simultaneous equations ... Are consumers responding to the advertising or are advertisers responding to the consuming?”

Comprehensive literature reviews of the effect of advertising on tobacco consumption indicate that the evidence is mixed as to whether advertising increases use or has no detectable effect. Blecher (2008) identifies 18 studies that find no significant effect of advertising on smoking, and 17 analyses that uncover a significant positive impact. Saffer and Chaloupka (2000) classify studies of advertising and cigarette consumption according to whether the data are time series or cross-sectional. Among the time series studies, 9 find no effect of advertising and 6 find a small positive effect. All three cross-sectional studies examined indicate a positive effect of advertising.

Two meta-analyses of advertising elasticities of demand for cigarettes and alcohol find that use is less sensitive to advertising than to prices or income. Gallet and List (2003) located 137 published estimates of the advertising elasticity of cigarette smoking, the mean of which is 0.10, with a standard deviation equal to 0.13 and ranging from -0.10 to 069 . Gallet (2007) located 132 studies containing 322 estimated advertising elasticities of alcohol consumption, of which the median estimate is 0.029 .

Chou, Rashad, Grossman (2008) merge data from the 1979 and 1997 National Longitudinal Surveys of Youth (NLSY79 and NLSY97) with information on weekly hours of television advertisements for fast food restaurants by designated market area (DMA) and year. They estimate that if youths were exposed to an additional half hour of fast food advertising per week, the probability of being overweight would rise by 2.2 percentage points (15 percent) for boys aged 3-11 years, 1.6 percentage points (or 12 percent) for girls aged 3-11 years, 2.5 
percentage points (17 percent) for boys aged 12-18 years, and 0.6 percentage points ( 4 percent) for girls aged 12-18 years. This research is limited by a lack of information about fast food consumption (the outcome examined is BMI). Moreover, the child's exposure to advertising is estimated using the number of hours that the child reports watching television and the ads aired in that DMA; the researchers do not know how many and which fast food commercials each child saw. Moreover, advertising in the DMA may be endogenous; fast food restaurants likely target advertising to areas whose residents are expected to have a high demand for fast food. Controlling for DMA fixed effects accounts for time-invariant differences in demand but there remains potentially endogenous variation in demand over time within DMAs.

Saffer and Dave (2006) pursue a similar strategy, merging individual-level data from the Monitoring the Future and NLSY97 with market-level data on alcohol advertising in television, newspapers, radio and outdoor media. They examine the 75 largest DMAs in the U.S. and find that advertising is positively, although modestly, correlated with the probability of alcohol use and binge drinking. They acknowledge that the results may be biased if advertising expenditures are a function of factors affecting demand for alcohol. This study also suffers the limitation of not being able to accurately estimate exposure to ads within DMAs.

Virtually all previous studies examining the impact of advertising on health behaviors are limited by the likelihood that advertising exposure is endogenous, e.g. through targeting of ads to consumers likely to demand the products. A recent review of the literature examining the impact of cigarette marketing on smoking criticizes this work for failing to address the endogeneity of marketing exposure and concludes that the findings "fall far short of those required to establish well-founded causal relationships.” (Heckman et al., 2008, p. 43). The study that best estimates the effects of advertising exposure, while addressing the endogeneity of exposure, is Avery et al. 
(2007). Using the Simmons National Consumer Survey, the authors merge data on the number of advertisements for smoking cessation products in the specific magazine issues read by the respondent. To control for the selective targeting of ads, the authors control alternately for categories of magazines read (so variation in advertising exposure comes from, for example, reading Time instead of Newsweek) and for magazine fixed effects, thus exploiting variation over time in the number of ads in each magazine. The authors consistently find that exposure to magazine advertisements for smoking cessation products raises quit attempts, with weaker evidence of increases in successful quitting. ${ }^{35}$

\section{g. Time Preference and Health Behaviors}

The rate of time preference refers to an individual's willingness to exchange utility today for utility later: it is the marginal rate of substitution between current and future utility (Becker and Mulligan, 2001). Suppose that an individual seeks to maximize the present discounted value of lifetime utility:

$$
U=\sum_{t=1}^{T} \delta^{\mathrm{t}}\left(\mathrm{U}\left(\mathrm{C}_{\mathrm{t}}\right)\right) \text { for } \delta=\frac{1}{1+\sigma},
$$

where $\mathrm{U}\left(\mathrm{C}_{\mathrm{t}}\right)$ is utility in period t. A higher rate of time preference $\sigma$ indicates that the person is less patient (to a greater extent prefers utility today to utility tomorrow). The discount factor $\delta$ has the opposite correlation: a smaller discount factor implies less patience (future utility receives a weight closer to zero) and a higher discount factor implies greater patience (a higher weight for utility in later periods).

Victor Fuchs (1982) was one of the first economists to examine the relationship between rate of time preference and health behaviors, motivated by the large literature documenting a

\footnotetext{
${ }^{35}$ As a falsification test, they check whether future advertisements for smoking cessation products (in the same magazines the respondent currently reads) affect the current probability of quitting, and find that they do not.
} 
positive correlation between education and health. Fuchs (1982) argues that the correlation of education with good health could reflect differences in rate of time preference. Patient individuals are more likely to forego current utility in exchange for long-run benefits; this is likely to result in healthier behaviors (e.g. more exercising) and higher education, even if schooling has no causal effect on health. Fuchs finds that rate of time preference (elicited from questions about willingness to exchange a certain amount of money today for a larger amount in the future) is positively correlated with schooling and usually also with healthy behaviors, although the point estimates are often small and not always statistically significant.

Time preference is notoriously difficult to measure. Most of the empirical literature attempting to do so uses one of two approaches. The first is to infer a discount factor from Euler equations of consumption (e.g. Lawrance, 1991) or wealth (e.g. Samwick, 1998) in different periods. A limitation of this approach is that identification of the rate of time preference is dependent on strong assumptions about functional form (Lawrance, 1991; Zhang and Rashad, 2008). A second common method is to survey respondents using hypothetical scenarios regarding willingness to exchange money today for (more) money in the future (e.g. Fuchs 1982, Fuchs and Farrell 1982). This approach is limited because people may not provide accurate answers to hypothetical scenarios and responses to such questions may instead measure expectations about interest rates, rates of return on investments, or attitudes toward risk, rather than discount factors.

A fundamental challenge for all methods of calculating the discount rate is that rates of time preference may vary across types of consumption. For example, an individual may be happy to save rather than spend money, but eager to defer physical pain rather than to experience it immediately. Moreover, the marginal rate of substitution for consumption in two particular 
periods may change over time or across the lifecycle. ${ }^{36}$ These issues will be discussed in detail in section 3.

Becker and Mulligan (1997) model time preference as endogenous. They point out that there is an incentive to reduce the rate of time discount because doing so raises the present discounted value of lifetime utility. They, along with Fuchs (1982), hypothesize that schooling may provide a method of decreasing one's discount rate and that this provides a possible mechanism through which education improves health. Becker and Mulligan further suggest that precommitment mechanisms (such as "Christmas Clubs” that enforce saving) may be investments in learning patience. Parents may invest in reducing their children's discount rates, so that the youth will be more willing to make investments that involve short-term costs but long-term gain, which can yield benefits for health, human capital, and wealth. In the addiction model of Orphanides and Zervos (1998), discussed above, consumption of addictive substances raises the rate of time preference (reduces patience) but individuals are aware of this and account for it when making consumption decisions.

Fuchs and Farrell (1982) find that among white, non-Hispanic adults with between 12 and 18 years of schooling, in the Stanford Heart Disease Prevention Program, the negative correlation between eventual completed schooling and smoking is as strong at age 17 (when all were in the same grade, and differences in education had not yet arisen) as at age 24 (when they differed in attained education). Based on this, Fuchs and Farrell (1982) reject the hypothesis that years of schooling reduce smoking and conclude that omitted variables explain the observed correlation. They are unable to test which omitted variables are responsible, but hypothesize that one is rate of time discount.

\footnotetext{
${ }^{36}$ See Frederick, Loewenstein and O’Donoghue (2002) for a general discussion of these issues.
} 
Several recent papers demonstrate a correlation between body mass index (BMI) or obesity and proxies for rate of time preference such as savings rates (Komlos et al., 2004; Smith et al. 2005) or willingness to delay financial rewards or other gratification (Borghans and Golsteyn, 2008; Ikeda et al., 2010). Conversely, Chapman et al. (2001) find weak or no association between health behaviors (influenza vaccination, adherence to medication for high blood pressure, adherence to medication for high cholesterol) and time preference assessed using hypothetical scenarios. Khwaja et al. (2007) show that smokers and non-smokers have similar rates of discount when the latter is proxied by willingness to undergo a colonoscopy and conclude that variation in discounting is not a major explanation for differences in smoking behavior.

Cutler and Glaeser (2005) argue that if discount rate heterogeneity explains variations in health behaviors, we should observe high within-person correlations across health behaviors e.g. alcohol consumption should be higher among smokers than nonsmokers. They test for this using data for individuals 45 and older from the 1990 National Health Interview Survey on: current smoking, consumption of three or more alcoholic beverages per day, obesity, use of recommended hypertension medication, and (for women) receiving mammograms in the past three years. They find that the correlations across health behaviors are "surprisingly low" (p. 238); most below 10\%, with the highest (alcohol and smoking) at 16\%. They obtain similarly weak correlations using data from the Behavioral Risk Factor Surveillance System on smoking, drinking, obesity, seatbelt use, flu shots in the past year, and cancer screening. Changes over time in health behaviors (smoking, heavy drinking, being overweight, and physical inactivity) are also weakly correlated (in the Health and Retirement Study). While these results suggest that time preference is not a major determinant of health behaviors, other explanations are that these 
behaviors are primarily substitutes (rather than complements), and myopia about future consequences leads people to become addicted to certain unhealthy behaviors but not others.

\section{Alternative Approaches to Studying Health Behaviors}

\section{a. Peer Effects}

In the basic model of health capital, individuals make decisions in isolation from each other. Manski (2000) notes, however, that there are three channels through which individuals may affect each other. The first is through constraints in shared resources. For example, there may be only so many treadmills at the gym, or so many roster spots on the school sports team, and as a result one individual's decision to exercise or play sports can prevent another from doing the same. Second, individuals may influence each other's behavior through expectations. For example, teenagers may update beliefs about the marginal benefits and costs of risky behaviors by discussing their sexual experiences or observing each other's drug use. Third, individuals may directly affect each other's preferences.

This third mechanism is the focus of Leibenstein (1950). He emphasizes the role of "bandwagon” effects (deriving utility from consuming the same goods and services as peers), which makes the demand curve more elastic because, when price falls, demand increases both directly (because of lower prices) and indirectly (because others are more likely to be using the good). Conversely, "snob" effects work in the opposite direction. When something becomes common, people may not want to consume as much of it (perhaps because it no longer signals exclusivity). Snob effects make the demand curve less elastic because the increased consumption associated with a price decrease makes the good less desirable to consume. Bandwagon and snob effects imply that the market demand curve is not simply the horizontal 
summation of all individual demand curves in the market and that peer interactions matter in ways not captured by simple economic models.

For many addictive goods, consumers may strike a balance between bandwagon and snob effects. For example, teenagers may wish to rebel against the majority (consistent with a snob effect), but probably not in an utterly unique way that leaves them isolated from everyone else, so they choose emulate a small subset of peers, consistent with a limited bandwagon effect.

A rapidly growing empirical literature has investigated peer effects in health behaviors. Manski (2000) notes three possible explanations for the correlation of behaviors within groups: 1) endogenous interactions, where behavior of the group affects behavior of the individual; 2) contextual interactions, where exogenous characteristics of group members (such as age or family background) affect behavior of the individual; and 3) correlated effects, a nonsocial effect in which the group behaves similarly because they have similar characteristics or environment. For example, smoking may be correlated within youth peer groups for any or all of these reasons. There may be endogenous interactions such as peer smoking increasing own tobacco consumption because smoking is a bandwagon activity. There may be contextual interactions if teens who hang out with older kids tend to have smoking peers (because the peers are older), and having older friends (whether smokers or not) is associated with more frequent initiation of smoking. Finally, there may be correlated effects in the sense that low-income youth tend to hang out together, and low incomes are associated with smoking initiation.

The source of correlated group behaviors has important implications for public policy. For example, smoking cessation programs targeted to individual teens may have spillover effects to peers if there are endogenous interactions, but not if there are only contextual interactions or correlated effects. 
Manski $(1993,2000)$ emphasizes the difficulty in empirically distinguishing between these effects because of the "reflection problem": the observed correlation between an individual and his peers is a composite of both the impact of peers on the individual and of the individual on the peers. Researchers have sought to overcome the reflection problem by: assuming a specific length of lag between mean group behaviors and those of the individual, modeling individual behavior as a specific nonlinear function of group behavior (i.e. assuming that individuals are responsive to some feature of the group distribution of behavior other than the mean), or using instrumental variables approaches with instruments that explain exogenous variation in either the behavior of group members or the membership of the peer group. Even if endogenous interactions can be proven, Manski (2000) argues that such a finding is only useful if one can demonstrate that the mechanism is preferences (e.g. the stigma of drug consumption falls when its use rises), expectations (e.g. youths learn how pleasurable drugs can be by seeing others enjoy them), or constraints (e.g. search costs for drugs are reduced when friends buy and use them).

The nature and variety of empirical investigations on peer effects can usefully be illustrated by focusing on studies of obesity, which have received considerable attention, particularly in response to an examination of data on adults from the Framingham Heart Study indicating that obesity spreads within social networks (Christakis and Fowler, 2007). That study does not exploit any exogenous variation in either peer group membership or in the behavior of peers; instead they attempt to control for correlated effects by controlling for lags of both respondent obesity and peer obesity in a model that regresses respondent contemporaneous obesity on peer contemporaneous obesity status. Key findings are that the chances of becoming obese rose by $57 \%, 40 \%$ and $37 \%$ respectively if the respondent had a friend, sibling, or spouse 
who became obese. These findings could reflect selection (friends and spouses choosing each other based on future weight or weight trajectories), correlated effects due to shared environments (such as local food prices and availability, availability of exercise opportunities), or true endogenous interactions (i.e. causal peer effects). Christakis and Fowler (2007) and Fowler and Christakis (2008) argue that there is likely to be a true peer effect because the estimated effects are stronger for pairs who both list each other as friends than pairs in which one but not both individuals claim friendship. They also find that geographic proximity of peers does not matter - the authors interpret this second result as ruling out a common environment effect. However, even for true endogenous interactions, one might expect proximity to be important if the size of the peer effect depends on the frequency of interactions with the peer.

Cohen-Cole and Fletcher (2008a) investigate the sensitivity of Christakis and Fowler (2007) results using a different dataset (the National Longitudinal Survey of Adolescent Health or Add Health) and age group. ${ }^{37}$ When using the same regression model, they largely replicate the findings of Christakis and Fowler; however, when controls for school-specific time trends (as a proxy for the environment) included, the peer correlations fall more than $30 \%$, suggesting the importance of omitted group-level characteristics. ${ }^{38}$ In addition, they conduct falsification tests, using the Add Health data, showing that the Christakis and Fowler's (2007) regression model generates apparent network effects for outcomes for which true peer effects are unlikely (e.g. acne, height, and headaches) and that these disappear after controlling for environmental confounders. ${ }^{39}$ They conclude that the method used by Christakis and Fowler (2007) is not

\footnotetext{
${ }^{37}$ Add Health is a nationally representative sample of $7-12^{\text {th }}$ graders first interviewed in 1994-95.

${ }^{38}$ However, Christakis and Fowler (2008) interpret this work as supporting their own, pointing out that their original estimates are within the 95\% confidence intervals of Cohen-Cole and Fletcher (2008a).

${ }^{39}$ Falsification tests apply the empirical methods to outcomes where the hypothesized relationships should not occur. Large or stastically significant associations then suggest problems with the empirical strategy. In a classic falsification test, Dranove and Wehner (1994) used a standard method of testing for demand inducement for medical
} 
sufficiently specific to separate true network effects from spurious correlations due to insufficient controls for the local environment.

Identifying the correct peer group is a challenge. "However severe the reflection problem may be when group composition is known, the problem becomes insurmountable when group composition is unknown” (Manski, 2000, p. 129). Moreover, peer groups may vary across outcomes; for example, a teenage boy might experience bandwagon effects for physical fitness from his sports teammates, for risky sexual activity from his classmates, and for alcohol from his older brother. In practice, researchers examining health behaviors have examined a variety of peer groups, almost always driven by their opportunistic availability in secondary data, including: classmates (Lundborg, 2006; Argys and Rees, 2008), friends (Christakis and Fowler, 2007; Cohen-Cole and Fletcher, 2008), siblings (Christakis and Fowler, 2007), spouses (Christakis and Fowler, 2007), neighbors (Christakis and Fowler, 2007; Case and Katz, 1991), and college roommates (Yakusheva, 2010; Duncan et al., 2005). ${ }^{40}$

The method of instrumental variables can be applied to estimate peer effects by utilizing exogenous variations in peer behavior. For example, Trogdon et al. (2008) and Renna et al. (2008) instrument for the weight of a peer using the obesity status of the peer's parents. The validity of these instruments is questionable, however, because friendships could also be selected on the basis of obesity status, with obese youths are relatively likely to have obese parents. This strategy may also suffer from a second-order case of the reflection problem - friend's parents'

care in a situation where it should not hold: pregnancies. Their finding that obstetricians/gynecologists were estimated to increase the number of pregnant women suggests shortcomings of the standard test.

${ }^{40}$ Other relevant peer groups may include an online community or adolescents seen on television. In Christakis and Fowler (2007), "friends" were those that respondents listed as being able to get in touch with them in case they had moved and the surveyors was unable to find their new address or phone number. These might be friends or merely those most likely to know their whereabouts. Add Health asked respondents to list their five closest male and five closest female friends. This survey also contains information on a large number of students in the same schools, allowing researchers to use classmates as another peer group. In both the Framingham and Add Health data, one is able to explore the importance of symmetry: do two respondents list each other as friends, or does one person list the other as a friend but it is not reciprocated? 
weight may be affected by friend weight which in turn may be affected by the respondent's weight.

A second strategy for identifying causal peer effects is to instrument for peer behavior using exogenous variation in peer group membership; in the previous literature this has most frequently concerned classmates or roommates. For instance, Argys and Rees (2008) use birth date relative to the cutoff for starting kindergarten to generate exogenous variation in the age of the youth relative to classmates; they find that females with older peers are more likely to use substances (marijuana, alcohol, tobacco) but with few peer effects for boys. Lundborg (2006) assumes that while schools may be chosen by parents, the specific classroom within a grade is randomly assigned; utilizing across-classroom differences in peer behavior by controlling for school and grade fixed effects, he finds effects of classmates on binge drinking, smoking, and use of illicit drugs. Duncan et al. (2005) use randomized roommate assignment and find that boys who binge drank in high school consume more alcohol in college if their roommate binge drank in high school. No such peer effects were found for boys who didn’t binge drink in high school, or for girls, or for marijuana use or sexual behavior for either boys or girls. Yakusheva et al. (2009) also exploit random roommate assignment and find that female college students gained less weight during freshman year when their roommate was heavier. Carrell et al. (2010) exploit random assignment of United States Air Force Academy cadets to squadrons (of approximately 30) with whom cadets live, eat, study and compete in intramural sports. They find substantial peer effects; e.g. that the effect on current fitness of friends' high school fitness is nearly $40 \%$ as strong as the effect of own high school fitness. These peer effects are caused primarily by friends who are least fit; this may be due to the sample consisting of unusually fit individuals who may not have much room for improvement. 
In the Moving to Opportunity experiment, adults receiving a waiver to move to a higherincome neighborhood were 5 percentage points less likely to be obese than similar persons not receiving a waiver (Kling et al., 2007); this intent-to-treat estimate is consistent with those moving to more advantaged neighborhoods adopting a new set of peers with healthier habits. An alternative explanation is that the new neighborhoods better facilitate healthy eating and physical activity.

Another general empirical challenge in the peer effects literature is correctly modeling which aspects of the distribution of behavior in the peer group are relevant. Most analyses model individual behavior as a function of the central (especially average) tendencies of the peer group, but the presence or absence of extreme actions could be more important. High outliers ("bad apples”) and low outliers ("straight arrows") might be especially influential on behavior. For example, the probability that a female college student develops an eating disorder might be influenced by the number of sorority sisters who are underweight, rather than average body weight of sorority members.

\section{b. Information Constraints}

Individuals may make lack the information needed to accurately assess the costs and benefits associated with various health behaviors. One important question is whether individuals understand how health behaviors alter the risks of morbidity and mortality. If individuals underestimate the risks associated with unhealthy behaviors, government intervention to either directly provide the missing information or require disclosure of information by producers could be warranted. Kenkel (1991) provides evidence that health knowledge is related to smoking, drinking and exercise in the expected directions, but that even some highly knowledgeable persons have poor health habits. 
A large economics literature has examined consumer awareness of, and sensitivity to, information on the health consequences of smoking. Smoking is especially informative because its risks are well understood and have been widely publicized since the 1964 Surgeon General's report (U.S. Department of Health, Education and Human Welfare, 1964).

A landmark study by Viscusi (1990) found that adults were much more likely to overestimate than to underestimate the extent to which smoking raises the risk of lung cancer. Specifically, he estimated that the true lifetime risk of lung cancer for smokers was between 5 and 10 percent but respondents to a national telephone survey estimated that the risk was 43 percent. Assuming the true risk was 10 percent, approximately 90 percent of the sample overestimated the risk, with 51 percent thinking it exceeded 50 percent. Smokers had lower risk estimates than non-smokers but still overstated the true risk fairly dramatically - the average stated subjective risk was 37 percent; 86 percent estimating greater than a 10 percent lifetime risk for smokers and 42 percent putting the odds above 50 percent. These risks are overestimated even if respondents’ answers were based on all of risks of smoking (e.g. including those from heart disease, strokes and emphysema).

Analyses of data from the Health and Retirement Survey (HRS) reach a starkly different conclusion. Schoenbaum (1997) finds that 50-62 year old heavy smokers had expectations of living to age 75 that were almost twice as high as actuarial predictions. Kwaja et al. (2007), using the same data, find that, for the sample as a whole, subjective beliefs about survival were similar overall to the objective data but that current smokers were overly optimistic about survival while those who had never smoked were relatively pessimistic. ${ }^{41}$ Smith et al. (2001)

\footnotetext{
${ }^{41}$ Objective survival probabilities were based on mortality within the HRS sample, rather than on life tables. , Khwaja, et al. (2009) find that 50-70 year olds relatively accurately predict their probability of survival to age 75 regardless of their smoking status.
} 
estimates that heavy smokers are more optimistic about their self-assessed longevity than their smoking behavior would warrant. ${ }^{42}$

One explanation for the difference in the aforementioned findings is that individuals may suffer from optimism bias; i.e. they may have accurate knowledge of population risks but still underestimate their personal risk. Viscusi’s (1990) question “Among 100 cigarette smokers, how many do you think will get lung cancer because they smoke?” may measure knowledge of risks to the general population, whereas the HRS data analyzed by Schoenbaum (1997) and Kwaja et al. (2007) contained respondents beliefs that they personally would live to age 75.

Supporting this possibility, Smith et al. (2001) show that smokers and non-smokers in the HRS respond to health information differently: smokers dramatically reduce their subjective life expectancies when confronted with smoking-related health shocks but decrease their subjective life expectancies less than than non-smokers in response to non-smoking related health shocks. The authors conclude that smokers may not personalize the risks of tobacco use unless there is clear evidence that it is negatively affecting their own health. If true, this implies that general information (such as that required by labeling laws) may have only a limited effect on behavior until individuals incur a health shock related to the unhealthy behavior.

\section{c. Time-Inconsistent Preferences and Hyperbolic Discounting}

Models of intertemporal choice have typically been based on the exponential discounting model of Samuelson (1937); e.g. the Samuelson framework is the basis for the optimization problem in the rational addiction model discussed above. A key feature is that the discount rate between any two periods $t$ and $t+1$ is a constant (d), implying that the discount rate between

\footnotetext{
${ }^{42}$ Similarly, in an interesting analysis of secondary life insurance markets (viatical settlements) for individuals with HIV/AIDS, Bhattacharya et al. (2009) provide evidence that relatively healthy individuals understate their remaining life expectancy while those who are relatively unhealthy overstate it.
} 
periods $t$ and $t+n$ is $d^{n}$, for all $n$. Such behavior is often referred to as "time-consistent" since the marginal rate of substitution for consumption in any two periods remains constant over time.

Exponential discounting quickly became standard in models of intertemporal decisions because it provides a straightforward method of extending single-period utility-maximization into a multi-period context, not because it accurately depicts the way that such decisions are actually made. To the contrary, as discussed below, individuals often have "time-inconsistent" preferences, e.g. discount rates are higher for intertemporal tradeoffs that occur in the near future than for longer time horizons. This is called "hyperbolic” discounting (Ainslie, 1991). ${ }^{43}$

A key implication of hyperbolic discounting is "present-biased” preferences; the tradeoff between utility in the current versus the next period is greater than that for any two adjacent periods in the future. Hyperbolic discounting results in time-inconsistent behavior. For example, assume a person is willing to trade one "util" of happiness in period $\mathrm{n}$ in the future for two utils received in period $n+1$. With hyperbolic discounting and stable preferences, a person who is willing to make that trade when periods $n$ and $n+1$ are in the distant future will also be willing to make that trade when it actually is period n. However, with hyperbolic discounting, there may be a preference reversal; the person might be willing to trade one util in period $\mathrm{n}$ for two utils in period $n+1$ while those periods were in the distant future, but when it actually is period $\mathrm{n}$ the individual might suddenly decide that more than two utils would be needed in the next period to compensate them for giving up a util immediately. Consider an individual who is choosing on Monday how much ice cream to eat on Friday. An exponential discounter will carry out on Friday the plans made on Monday (assuming no changes in income, prices, or other relevant variables) because preferences are time consistent. However, a hyperbolic discounter may plan on Monday to skip the ice cream, but when Friday comes, she may experience a

\footnotetext{
${ }^{43}$ This idea was first formalized by Strotz (1956).
} 
preference reversal, suddenly being unwilling to deny herself the current utility from consuming the ice cream. Thus, time-inconsistent preferences lead to self-control problems and future plans to engage in healthy behavior (in the future in order to maximize the present discounted value of lifetime utility) are consistently undone by (abnormally) high present discount rates.

Time-inconsistent preferences can affect many health behaviors. Plans made the night before to exercise in the morning will not be realized when the alarm clock goes off, intentions to consume alcohol in moderation will be undone as the immediate pleasures of having "just one more drink" are repeatedly acted upon, and so forth. Individuals who are naive about their time inconsistent preferences may be endlessly optimistic about their ability to improve future health behaviors. Those who are more sophisticated may seek precommitments that compel their future self to adhere to healthy behaviors. For instance, they may avoid bringing home ice cream, knowing that doing so will result in overeating. Or they may plans to run with a friend, aware that this will make it harder for them to skip the workout. Each of these tactics is designed to ensure that binding decisions are made when the marginal rate of substitution between two future periods is relatively low, rather than later, when the marginal rate of substitution will change to incentivize immediate gratification. ${ }^{44}$

By far the most common way that economists have modeled hyperbolic discounting is using the quasi-hyperbolic (or $\beta-\delta$ ) framework developed by Laibson (1997), based on the functional form first used by Phelps and Pollak (1968) in their study of optimal intergenerational savings. Specifically, the utility function is characterized by:

$$
U_{t}=\mathrm{u}\left(\mathrm{C}_{\mathrm{t}}\right)+\beta \sum_{n=1}^{T-n} \delta^{t} \mathrm{u}\left(\mathrm{C}_{\mathrm{t}+\mathrm{n}}\right)
$$

\footnotetext{
${ }^{44}$ Sophistication is a double-edged sword. O'Donoghue and Rabin (1999) point out that sophisticated hyperbolic discounters are more likely than naive hyperbolic discounters to develop precommitment strategies but may also more frequently "preoperate" - realizing that they are unlikely to be able to stick to their plan, they abandon it earlier. For example, a sophisticated hyperbolic discounter may realize that she is unlikely to be able to stick to her diet and may cease even trying, whereas a naive hyperbolic discounter may naively start diet after diet.
} 
where $\mathrm{u}(\cdot)$ is utility in the specified period, $\mathrm{C}$ is a composite consumption good, $\mathrm{T}$ is the time horizon over which utility is measured (known with certainty), with $\beta \leq 1$ and $\delta \leq 1$. The key implication is that the discount factor for consumption between period $t$ and $t+1$ is $\beta \delta$, whereas that between any two future periods, $t+j$ and $t+j+1$ (for $j>0$ ) is $\delta$. If $\beta=1$ this reduces to exponential discounting. However, if $\beta<1$, the discount factor is lower (and the discount rate higher) for immediate than future consumption tradeoffs. This results in time-inconsistent preferences and self-control problems similar (but not identical) to those in more general forms of hyperbolic discounting and that can be relatively easily incorporated into standard economic models of intertemporal choice.

Substantial research suggests that hyperbolic discounting explains many real-world decisions more accurately than standard exponential discounting. For example, Thaler (1981) found that the future payments required to make individuals indifferent between receiving a prize now versus later yielded implied (per period) discount rates that declined dramatically as the length of time increased, consistent with hyperbolic discounting. ${ }^{45}$ Angeletos, et al., (2001), and the references contained therein, indicate that a wide variety of time preference experiments show that decision-makers are more impatient in the short-run than the long-run. Frederick et al. (2002) examine a large number of empirical studies and find that discount factors increase (discount rates decrease) as the time horizon of the study increases but that this relationship disappears when studies covering less than one year are eliminated. Such results are consistent with hyperbolic discounting. ${ }^{46}$

Gruber and Köszegi (2001) incorporate time-inconsistent preferences in a model of smoking and distinguish between sophisticated and naïve agents, where the former understand

\footnotetext{
${ }^{45}$ Discount rates also declined with the size of the prize and losses were discounted differently from gains.

${ }^{46}$ There is also clear evidence that animals discount hyperbolically (e.g. see Berns et al., 2007).
} 
that their preferences are time-inconsistent but the latter do not. A key prediction is that future price increases are predicted to reduce current consumption. Because this is generally the key test for rational addiction, standard econometric tests (focusing on responses to future changes) cannot distinguish between the TORA and similar frameworks with time-inconsistent preferences. $^{47}$ Cutler et al. (2003) also use an informal model with hyperbolic discounting to consider (but not formally test) how time-inconsistent preferences affect eating decisions and obesity, and the resulting consequences for social welfare. ${ }^{48}$

\section{d. Cognitive Limitations and Bounded Rationality}

Hyperbolic discounting does not change the utility maximization assumptions standard in economic models, only the method of discounting. However, it is possible that individuals cannot (or for whatever reason, do not) maximize utility when faced with highly complex problems. Such limitations are the basis of the models of bounded rationality and "satisficing" developed by Herbert Simon (1984) and applied by economists to many decision processes (e.g. see Thaler and Sunstein, 2009), including those related to health behaviors.

For instance, in Suranovic et al.’s (1999) modified rational addiction framework, an individual decides how much to smoke (S) at age t by maximizing:

$$
\mathrm{U}_{\mathrm{t}}(\mathrm{S})=\mathrm{B}_{\mathrm{t}}(\mathrm{S})+\mathrm{L}_{\mathrm{t}}(\mathrm{S})+\mathrm{C}_{\mathrm{t}}(\mathrm{S})
$$

where $\mathrm{B}$ is the current benefit of smoking, $\mathrm{L}$ is the fully discounted future loss from smoking, and $\mathrm{C}$ is the adjustment cost to changing tobacco use from recent levels. The key assumptions are that most utility losses occur at or near the end of life (and so are discounted heavily at young ages) and that adjustment costs are zero for smoking at or above recent levels but positive for

\footnotetext{
${ }^{47}$ Differential responses to price changes occurring at two or more future periods could theoretically be used to test for the existence and amount of time-inconsistency, however, the data requirements to perform this exercise are severe.

${ }^{48}$ Their model emphasizes the secular decline in time spent on food preparation.
} 
lower amounts of use. ${ }^{49}$ The model is forward looking, in that future health costs are accounted for, but myopic in that the consequences of current smoking on future adjustment costs are not considered. It allows for several realistic consequences that are either difficult to explain or require strong assumptions using models with fully rational addiction. For instance, gradual rather than "cold turkey" withdrawal may occur if addiction is "weak" (i.e. if adjustment costs for deviating from past history rise at an increasing rate), and quitting may become more likely late in life, since the number of periods until losses are incurred decreases so that these are discounted less heavily. Importantly, by failing to fully account for the consequences of current smoking on future quitting costs, individuals may enter a consumption "trap," strengthening the potential case for policy intervention.

Akerlof (1991) provides a sophisticated analysis where bounded rationality leads to choices that separately are close to utility-maximizing but, in combination, result in large errors. Key aspects of his formulation are that decision-makers are slightly biased toward present rather than future utility (as described above) in ways that they are either unaware or do not fully account for. The result is that they avoid large mistakes at any single point in time but may make small errors that accumulate across periods.

Procrastination is a key example of such behaviors. Individuals may intend to start an exercise program or stop smoking at a date in the near future but time-inconsistency repeatedly prevents these intentions from being realized. Akerlof's analysis also emphasizes the role of peer influences in encouraging these decision errors, particularly in situations, as with gangs or cults, where the social milieu may be constructed to encourage initially small but cumulatively large changes in behaviors.

\footnotetext{
${ }^{49}$ By contrast, Dragone (2009) has developed a fully rational model of eating in which there are costs to any changes (either positive or negative) in food consumption. Under specified assumptions, overshooting will cause individuals to oscillate between gaining and losing weight, before eventually converging on the steady-state.
} 
In Rubinstein’s (2003) model of bounded rationality, agents simplify choices by applying "similarity relations." Specifically, when considering uncertain and multi-dimensional outcomes, individuals do not fully account dimensions of the choices that are "similar" but instead focus on those characteristics that are dissimilar. Consider lotteries of the form (X, p), in which a payout $\mathrm{X}$ is won with probability $\mathrm{p}$ and a payout of zero is won with probability 1-p. Rubinstein argues that, faced with the choice between $(\$ 3000,0.25)$ and $(\$ 4000,0.2)$, the difference in the size of the prize will be the decisive factor because the probabilities ( 0.20 and 0.25) will be interpreted as "similar" (and thus the difference will be ignored) whereas the winning amounts ( $\$ 3000$ versus $\$ 4000$ ) will be interpreted as dissimilar. ${ }^{50}$ He argues that this model explains some observed time-inconsistent behavior better than hyperbolic discounting. Specifically, he argues that individuals may perceive "10 years from now" and "11 years from now" to be similar, but perceive "today and "a year from now" as dissimilar.

Education is likely to be correlated with cognitive ability (which, when measures are available, usually contain error), possibly explaining some of the strong positive relationship between schooling and healthy behaviors noted in section 1 of this chapter. Education might also influence behaviors in other ways (e.g. by being correlated with or causally affecting discount rates, health knowledge, or access to high quality medical care) but an emerging body of research suggests that differences in cognition partly explain variations in health behaviors.

Cutler and Lleras-Muney (2010) conclude that about 30 percent of the average educationgradient in a wide variety of health behaviors in the United States and Great Britain is related to disparities in cognitive ability, with high-level processing being more important than measures of memory. Interestingly, cognitive ability at later ages is more important than that measured

\footnotetext{
${ }^{50}$ Conversely, when choosing between $(\$ 3000,1)$ and $(\$ 4000,0.8)$ both the prize amounts and probabilities are dissimilar so that other criteria (like maximizing expected payouts) will be used.
} 
earlier in life, suggesting that cognitive skills are learned (and helping to rule out explanations based on factors that are confounded with education and cognitive skill levels at a point in time). Similarly, using Australian data, Antsey et al. (2009) find that cognitive skill (proxied by verbal ability and processing speed) is correlated with vitamin and mineral supplement use, high rates of physical activity and light/moderate alcohol use, and reduced smoking. ${ }^{51}$

Chen and Lange (2008) and Lange (2010) examine how education is related to breast, colorectal and cervical cancer screening, with particular attention paid to differences between objective versus subjective cancer risk. A key result is that subjective risks of highly educated individuals more accurately reflect objective risks than do those of counterparts with less schooling, and that differences in subjective risks are more closely linked to screening decisions for them. These education differences do not appear to occur because of a positive correlation between schooling and income or the quality of medical care but could reflect the more scientific world-view of those with more schooling. One overall conclusion is that the highly educated are better at processing information related to medical risks and the behaviors required to ameliorate them (although it is not clear that schooling causes these differences).

De Walque (2007a) demonstrates that a series of HIV/AIDS prevention campaigns in Uganda during the late 1980s and early 1990s resulted in larger reductions in the incidence of HIV infection for highly educated young men and (particularly) women than for their counterparts with less schooling, with increased condom use playing a key role. Although alternative explanations cannot be completely ruled out, the greater ability of highly educated

\footnotetext{
${ }^{51}$ This study does not identify the direction of causation.
} 
persons to process the information provided in these campaigns may represent a significant source of these schooling-related differences. ${ }^{52}$

Rosenzweig and Schultz (1989) show that education increases the ability of couples to successfully use complicated contraceptive methods (rhythm or withdrawal) but with no corresponding differences for simpler methods (e.g. the pill or IUD). Contraceptive effectiveness also increased with schooling following unplanned pregnancies. Both results suggest that increased cognitive ability favorably influences behaviors, particularly when information is limited or idiosyncratic.

Goldman and Smith (2002) show that education increases the probability that individuals with HIV or diabetes adhere to the complicated medical regimes developed to treat these diseases and that this superior health management is linked to better outcomes. ${ }^{53}$ Although this evidence refers to disease management, the same mechanisms seem likely to operate for health behaviors, especially since many of the measures of adherence (particularly for diabetes) were behavioral in nature..$^{54}$ They further show that the education differential in adherence to diabetes treatment largely disappears after controlling for scores on the Wechsler Adult Intelligence test (measuring high level abstract reasoning), further indicating the key role of cognitive skills. ${ }^{55}$

\section{e. Nontraditional Models}

\footnotetext{
${ }^{52}$ Similarly, De Walque (2007b) and Grimard and Parent (2007) find that education has a negative causal effect on smoking - using IV procedures exploiting college attendance as a strategy to avoid the Vietnam war - but they are not able to say whether the effect of schooling reflects increased cognitive processing abilities or other factors, such as education-related decreases in discount rates, improvements in access to information, or wage increases.

${ }^{53}$ Maitra (2010) confirms that the highly educated have better diabetes treatment adherence but raises questions about the extent to which this explains better self-reported health.

${ }^{54}$ For instance, Goldman and Smith (2002) show that highly educated diabetics self-monitored blood glucose and self-tested their blood or urine more frequently than their counterparts with less schooling.

${ }^{55}$ Less educated persons also have higher rates of undiagnosed diabetes, controlling for health insurance coverage (Smith, 2007), which is suggestive of a behavioral response although it could also reflect differences in access to care. Lleras-Muney and Lichtenberg (2005) show that educated individuals tend to use more recently developed drugs, particularly in cases where learning is required (e.g. when drugs a repeatedly purchased to treat a medical condition), and that these effects are unlikely to result from differences in insurance or access to medical care.
} 
The models described above deviate from standard economic optimization by adding constraints related to information or cognitive processing or by incorporating time-inconsistent preferences. However, attention has increasingly been paid, particularly in the areas of behavioral economics and neuroeconomics, to decision processes that differ more fundamentally from those traditionally used in economics. Kahneman (1994) distinguishes between "decision utility" and "experience utility". The key distinction is that individuals, when attempting to optimize based on decision utility, may incorrectly forecast the hedonic experiences (realized wellbeing) of different decisions (experience utility) and these errors may be systematic. Kahneman and Thaler (2006) offer the example of someone grocery shopping while hungry: she may buy overly large quantities of food because her current hunger leads her to overestimate the experience utility of eating in the future.

A possible reason for systematic errors is that decisions are influenced by immediate emotional experiences, called “visceral factors” by Loewenstein (2000), such as anger, fear, thirst, hunger, or sexual desire. Loewenstein argues that such factors have been traditionally discounted by economists because they fluctuate rapidly (although often in highly predictable ways) and because their impact is underestimated during “cool states” when individuals are not under their influence. In "hot” states, where visceral factors are operative, individuals "who otherwise display 'normal' decision-making behavior...behave in ways that give the appearance of extreme discounting of the future” (p. 430). ${ }^{56}$ In Laibson's (2001) cue-theory of consumption, repeated pairing of a cue and a consumption good eventually creates complementarity between the cue and consumption of the good (i.e. the presence of the cue raises the marginal utility of

\footnotetext{
${ }^{56}$ For example, Lerner and Keltner (2001) provide evidence that anger (but interestingly not fear) is associated with more risk-taking choices. Ariely and Loewenstein (2006) find that the willingness to engage in unsafe sex or in morally questionable behavior to obtain sexual gratification is higher for sexually aroused than non-aroused persons and that individuals poorly predict how sexual arousal will influence their behavior.
} 
consumption). He gives examples such as the smell of baking cookies and the sound of ice falling into a whiskey tumbler. Thus, cues can generate cravings in addicts and can be used in marketing to increase the consumption of food or alcohol. In contrast to the TORA, this model predicts high frequency variations in craving and marginal utility, which can lead to seemingly random patterns of consumption. (Although, Laibson notes that "cue effects can be captured using minor variants” of the TORA; see p. 82.) An important implication of the cue theory is that sophisticated consumers will actively engage in strategic cue management, which is consistent with the philosophy of (e.g.) Alcoholics Anonymous that its members must avoid people and locations (and of course, the sight or smell of alcohol) that could be cues that would lead to cravings and falling off the wagon. This theory also implies that there are negative externalities associated with cues, with consequent implications for policy to ensure that the production of cues does not exceed the level that would maximize social welfare.

A number of economists have used models of “multiple selves” to characterize decisionmaking processes. Thaler and Shefrin (1981) postulate that consumer behavior represents an internal battle between a farsighted "planner”, who values utility received in the distant future, and a myopic “doer” who prefers immediate gratification. The doer controls decisions but can be constrained by the farsighted planner through expenditures of (costly) willpower, (costly) precommitment devices restricting the choices available to or the tradeoffs faced by the doer, or other techniques for achieving self-control (e.g. rules, mental accounting, and framing). Similarly, Fudenberg \& Levine (2006) view decision problems as a game between a long-run patient self and a series of short-run impulsive selves. The long-run actor may again choose selfcontrol actions influencing the utility function of the myopic self, even though short-run costs must be incurred to reduce the future cost of self-control. Brocas and Carillo (2008) use a 
principal-agent approach in which the individual is split into a myopic but informed system (the agent) and a forward-looking but uninformed system (the principal) who maximizes the expected utility of all of the agents. Because the principal lacks complete information, she will optimally delegate certain choices to the agents but not others. For instance, the principal will offer the agent pairs of positively correlated labor supply-consumption choices that limit the consumption of "tempting” goods" but otherwise leave the agent free to make consumption decisions. For instance, this may take the form of strict prohibition on the consumption of addictive products, while allowing complete freedom in the use of those that are non-addictive. ${ }^{57}$

\section{i. Brain Structure and Decision-Making}

Many of the models just discussed link decision-making to the structure of the brain. In Thaler and Shefrin (1981), decisions of the "planner" primarily reside in the prefrontal cortex, while those of the "doer" occur in the more primitive limbic system (described below). Fudenberg and Levine (2006) similarly appeal to evidence that short-term impulsive behavior and long-term planned behavior occur in different parts of the brain. Brocas and Carrillo (2008) explicitly appeal to neuroscience evidence of multiple brain systems that split individual decision-making into two processes.

The following elements of brain anatomy provide insight into these characterizations. ${ }^{58}$

- The human brain evolved by adding new capabilities rather than replacing those previously existing. The brain stem and cerebellum, which developed first, control autonomic functions such as heartbeat and breathing. Surrounding this is the limbic system (the amygdale, thalamus, hypothalamus and hippocampus), which coordinates sensory inputs to generate

\footnotetext{
${ }^{57}$ In a somewhat related model of "temptation utility", Gul and Pesendorfer (2001) show that individuals may choose to limit the available choice set so as to avoid needing to exercise costly self-control.

${ }^{58}$ This discussion draws heavily on Ruhm (2010), which in turn is based on material in MacLean (1990), Massey

(2002), Bernheim and Rangel (2004), Loewenstein and O’Donoghue (2004), and Camerer et al. (2005).
} 
subjective feelings and drives states like anger, pleasure and aggression. The neocortex, which came last, consists of the occipital, parietal, temporal and frontal lobes (that deal with sensory processing), and the prefrontal cortex which is the locus of abstract thinking, conceptualization and planning.

- The limbic system responds to cues and stimuli without accounting for the long-term consequences of current actions, whereas the deliberative system, located in the neocortex, involves higher cognitive processes that do consider long-term consequences. The limbic and deliberative systems operate in parallel to yield differences in perception and memory, so that emotional feelings exist independently of rational assessments. We argue that many decisions about health behaviors involve an interaction of rational calculations with processes based on emotions, chemical responses, and feelings.

- The limbic system often acts upon external stimuli before deliberative processes take place in the neocortex. The number of neural connections running from the limbic system to the cortex also far exceeds those in the reverse direction, suggesting that emotional impulses frequently overwhelm cognitive processes.

Bernheim and Rangel (2004) draw on this neuroscience evidence in developing their model of cue-triggered decision processes. Specifically, they assume the brain contains a hedonic forecasting mechanism that learns from experience. However, the consumption of addictive substances interferes with this normal learning process by acting directly on the limbic system. Over time this system will be activated upon the presentation of the cues and the individual will enter a "hot" mode in which rational utility-maximization processes are bypassed. When the individual is in a "cold" mode, he makes decisions rationally with recognition of future 
consequences but in hot modes these cognitive processes do not operate. ${ }^{59}$ The sophisticated decision-maker is aware this occurs and rationally chooses a lifestyle that determines the possibility of being cued into hot modes.

This model differs from the TORA in a number of ways. Most importantly, consumption of the addictive good is frequently viewed to be a mistake because stochastic shocks (i.e. encountering cues that trigger hot modes) causes decisions to diverge from rationality. ${ }^{60}$ Cuetriggered addiction can also account for behaviors such as intentional use followed by halfhearted and later concerted attempts at abstention, or intentional recidivism.

One could alternatively model behavior as the result of multiple simultaneously-operating brain systems, without an extreme division into hot and cold modes. For example, in Loewenstein and O’Donoghue (2004), decisions reflect the interaction between the cognitively sophisticated deliberative system (located in the prefrontal cortex) and a rapidly responding affective system (occurring in more primitive brain structures). ${ }^{61}$ The affective system primarily controls behavior but the deliberative system exerts influence through the use of costly cognitive effort or willpower. ${ }^{62}$ Exposure to (potentially learned) cues and stimuli can trigger affective system responses but, in contrast to "hot/cold" models, the deliberative system almost always exerts at least some influence. The standard model of rational choice corresponds to the special case in which the deliberative system is in full control (i.e. exerting cognitive effort is costless) and hot states occur when the affective system is in full control (i.e. exerting cognitive effort has infinite cost).

\footnotetext{
${ }^{59}$ Metcalfe and Mischel (1999) provide an earlier and less formally developed "hot/cool” approach.

${ }^{60}$ Addiction also does not necessarily require adjacent complementarity in this model.

${ }^{61}$ Similar decision processes are modeled in the psychological literature without explicit linkages to brain function. For example, in cognitive-experiential self-theory, information is processed by "experiential" and "rational" systems that operate in parallel and are interactive: the experiential system is automatic, preconscious, rapid, and nonverbal; the rational system is analytic, deliberative, slow and affect-free (Epstein, 2003).

${ }^{62}$ Thaler and Sunstein (2009) refer to these as the "automatic" and "reflective" systems.
} 
This model provides insight into a variety of behaviors. First, individuals may simultaneously do one thing while wishing they were actually doing another (e.g. "I should not be eating this donut.”) Second, actions may frequently be influenced by transitory emotional states that result from affective system stimuli. Third, hyperbolic discounting and preference reversals occur naturally because future planning reflects deliberative processes but immediate decisions are strongly influenced by the affective system. Fourth, discount rates may vary across types of consumption (because affective system responses will be more important for some types than others) and situations (depending on how strongly the affective system has been triggered and how much willpower has been depleted). Fifth, the model can explain phenomenon such as loss aversion (if the affective system weights losses more heavily than gains) and non-linear probability weighting (individuals may consistently overestimate the probability of unlikely events and underestimate the probability of likely events) that are difficult to reconcile with standard utility-maximization.

Ruhm (2010) has developed and applied a variant of Loewenstein and O’Donoghue’s model in his examination of overeating and obesity. In his model, food consumption is influenced by the affective and deliberative systems and overeating has become more common over time partly because of lower food prices (as in traditional economic models) and partially because of the increased sophistication of "food engineering" whereby food products are increasingly designed to appeal to the affective system.

\section{ii. Behaviors Difficult to Explain Using Traditional Models}


Many aspects of generally observed behavior are difficult to reconcile with the traditional economic models in which decision-makers are fully rational. ${ }^{63}$ For example, Bernheim and Rangel (2004) point out that addicts typically describe their substance use to be a mistake even, in some instances, while they are in the act of taking the drug.

The data also suggest that the use of a single discount rate - whether exponential or hyperbolic - is unlikely to adequately describe many aspects of decision-making. For instance, Frederick et al. (2002) summarize a large body of evidence indicating that: gains are discounted more than losses (loss aversion); small amounts are discounted more than large amounts; improving sequences are preferred to worsening sequences; discount rates differ dramatically across situations and types of consumption. ${ }^{64}$ Many discounting "anomalies" can be explained if decision-making is based on multiple brain systems. Evidence that such brain modularity is important has been obtained from patients with brain damage. For example, persons with deficient limbic system function are less able to engage in gradual learning or to acquire conditioned responses to emotional stimuli, while individuals with damage to their prefrontal cortex exhibit impaired decision-making, with a particular inability to act on long-term goals (see Lowenstein and O’Donoghue, 2004; Camerer et al., 2005, or Brocas and Carrillo, 2008 for useful discussions of this literature). Some neuroscience evidence obtained from magnetic resonance imaging (MRI) supports the possibility that multiple-system models of decisionmaking arise directly from the structure of the brain (e.g. McClure et al., 2004); however, there remains ambiguity about these MRI results (e.g. see Glimcher et al., 2007).

\footnotetext{
${ }^{63}$ Although this section focuses on non-rational behavior, the consequences will, in practice, often be difficult to distinguish from non-exponential (e.g. hyperbolic) discounting. Either can cause, for example, self-control problems.

${ }^{64}$ Consistent with this, Cutler and Glaeser (2005) show that within-person correlations across health behaviors are quite low both at a point in time and when looking at behavioral changes across time. This variation results in part from genetic factors but behavior-specific situational influences are also important.
} 
The empirical data also suggest that the deliberative system exerts less power when cognitive processing resources are limited (when self-control is depleted) than when it is not. For example, Shiv and Fedorikhin (1999) describe an experiment in which subjects are asked to memorize either a two-digit or a seven-digit number and were then asked to choose a snack: either chocolate cake or fruit salad. Those who were asked to memorize a seven-digit number were more likely than those asked to memorize a two-digit number to request the chocolate cake (this was particularly true for those with high self-rated measures of consumer impulsivity), which the authors interpret as evidence that greater cognitive processing demands increases the likelihood that choices are driven by lower-order affective reactions rather than by higher-order processes like thinking or reasoning. More recently, Vohs et al., (2008) have shown that the cognitive effort involved in making choices reduces self-control among a variety of dimensions. $^{65}$

The frequent use of pre-commitment or other self-control devices is cited as evidence of time-inconsistent preferences of which agents have at least some awareness. For example, Gruber and Köszegi (2001) highlight the use of socially managed incentives to reduce smoking, such as announcing a New Year's resolution to quit smoking to create embarrassment for oneself if one resumes smoking. Ruhm (2010) emphasizes the increasing frequency of bariatric surgery, which can be viewed as an extremely strong pre-commitment strategy. The drug Antabuse is another market-generated precommitment device: by taking it in the morning an alcoholic can ensure that if he consumes alcohol later in the day he will be made ill by the interaction of the drug with the alcohol. A key distinction is that rational addicts may pay for technologies that help them quit or weaken their addiction but they should not be willing to pay to limit their

\footnotetext{
${ }^{65}$ They examine how making choices among consumer goods or college course options were related to physical stamina, persistence, procrastination and the ability to perform cognitive calculations. In each case, the group required to make choices experienced greater reductions in self-control or cognitive processing ability.
} 
future choices. Conversely, under alternative models, agents frequently make choices they will subsequently regret and so, under some circumstances, may choose to voluntarily constrain their future options.

Experimental and nonexperimental evidence suggests that individuals recognize their time-inconsistent preferences and act strategically to at least partially overcome them. Using data from three health clubs, Della Vigna and Malmendier (2006) compare the behavior of members to non-members, where the latter are allowed to use the clubs by paying for each visit. They find that members have higher per visit average costs and overestimate their future attendance. Those with monthly memberships are more likely, than annual members, to stay enrolled beyond one year (despite paying a higher fee for the flexibility to quit each month) and their attendance declines over time, in contrast to the increases observed for annual members. The researchers believe that overconfidence about future self-control provides the most likely explanation for these results.

Considerable ingenuity is required to distinguish between rational and non-rational use of addictive products. Most previous "tests" of the TORA identify forward looking behavior without distinguishing between complete or partial foresight, or between time-consistent preferences and self-control problems. Gruber and Köszegi (2001) emphasize that both the TORA and models of hyperbolic discounting imply responsiveness of current consumption to anticipated future price changes and the authors show that forward looking behavior by smokers need not indicate rational behavior. ${ }^{66}$

\footnotetext{
${ }^{66}$ Specifically, they find that announced future tobacco taxes have a positive effect on current cigarette sales, a result that suggests forward-looking behavior as consumers stock up prior to price hikes in order to save money. However, current consumption (not sales) appears to decline in response to future tax increases, as predicted by both TORA and less than fully rational models where consumers have some foresight.
} 
Because such direct tests are difficult to obtain, researchers have begun to use indirect evidence to distinguish between the two classes of models. Using data from the United States and Canada, Gruber and Mullainathan (2005) find that the happiness of smokers is increased when taxes are raised, consistent with sophisticated awareness of time-inconsistent preferences but probably not with rational addiction (because that model implies that higher prices reduce utility). Similarly, Kan’s (2007) IV estimates suggest that Taiwanese smokers who intend to quit are relatively supportive of smoking bans or cigarette tax increases. This is interpreted as evidence of a demand for self-control devices that are predicted with time-inconsistent preferences and forward-looking behavior. ${ }^{67}$ Ruhm (2010) tests a variety of predictions of a two-system model of overeating and obesity, with food engineering, against a standard model with full rationality. Among the most important are that the frequency of eating mistakes - as evidenced by weight loss attempts - and of the consumption of engineered foods (that are high in fat and salt) will have increased over time, particularly for heavy individuals, and that actual weight will have increased over time without an accompanying rise in desired weight. All of these predictions are born out using data for adults in the U.S.

\section{f. Short-Term Effects}

The discussion on health behaviors to this point has emphasized long-term factors and influences. (For example, education is anticipated to have lasting effects on decisions.) However, health behaviors are also influenced by short-term factors, including some that are poorly explained by many standard models. We discuss two examples below.

\section{i. Full Wallet Hypothesis}

\footnotetext{
${ }^{67}$ However, the appropriateness of the instruments (awareness of the health risks of smoking and weight loss attempts) is questionable quit attempts may increase the disutility of second-hand smoke, prompting support for these measures.
} 
According the permanent income hypothesis (PIH), short-term changes in income should have little influence on consumption decisions because spending is based "permanent" rather than current income (Hall, 1978). However, liquidity constrained individuals may not be able to smooth consumption as the model predicts and decision-makers with time-inconsistent preferences, or operating using the non-traditional models described above, may not even try to do so. The empirical evidence indicates that violations of PIH are common and that even very short-term changes in income affect a variety of types of consumption, including many health behaviors. This is sometimes referred to as the "full wallets" hypothesis.

Stephens (2003) showed that for persons receiving a major portion (at least 70 percent) of their income from Social Security, expenditures on "instantaneous consumption goods" (expenditures on food away from home and entertainment) increase by 33 percent on the day Social Security checks are received and by 35 percent on the next day, relative to average spending. The interpretation is that consumers have "full wallets" immediately after receiving their checks that results in higher spending because consumers are liquidity constrained at other times of the month. ${ }^{68}$ A similar responsiveness to the receipt of pay checks has been observed in the United Kingdom (Stephens, 2006) and to the receipt of food stamp benefits in the United States (Wilde and Ranney, 2000). ${ }^{69}$

Shapiro (2005) expands on this last result, showing that caloric intake declines 10 to 15 percent over the food stamp month. This is interpreted as evidence for quasi-hyperbolic discounting because, under exponential discounting, an annual discount rate of 146 percent

\footnotetext{
${ }^{68}$ Such behavior could occur without liquidity constraints if retailers discount prices at the beginning of the month (when Social Security checks are received). Instead, Hastings and Washington (2010) show that food prices decline slightly over the month (by about 3 percent between the first and fourth month) and that almost all of the change in food expenditures is due to variation in the quantities of food purchased.

${ }^{69}$ Energy intake also falls at the end of the "food stamp month" for those who shop infrequently.
} 
would be needed to explain these results. ${ }^{70}$ Mastrobuoni and Weinberg (2009) find even greater evidence of declining within-month food consumption for persons receiving 80 percent or more of their income from Social Security and who have little or no savings: annual discount factors are .08 for these persons, compared to flat consumption profiles for recipients with savings. The probability of consuming less than the recommended daily calories also increases dramatically toward the end of the benefit month for the former group but not for the latter.

The potential food insufficiency described above could have deleterious health consequences for at least some individuals. However, most detrimental consequences of shortterm changes in income appear to work in the opposite direction (i.e. "full” wallets are more harmful than "empty" wallets). Riddell and Riddell (2006) show that intraveous drug users are much more likely to be admitted to Vancouver hospitals with overdoses in the two days after welfare checks are received than at other times of the month. ${ }^{71}$ Dobkin and Puller (2007) obtain similar results for drug-related admissions, particular for cocaine overdoses, among California recipients of Supplemental Security Income (SSI) or Disability Income (DI). Interestingly, they do not find a corresponding "pay check" effect, suggesting that the consequences across population segments may be heterogeneous. ${ }^{72}$

Spikes in drug use due to full wallets have severe negative health consequences. Riddell and Riddell (2006) show that the death rates of SSI beneficiaries increase 22 percent on the day of benefit receipt - generally the first of the month. Mortality also increases at the beginning of the month for other reasons. Phillips et al. (1999) find that the overall U.S. death rate rises by

\footnotetext{
${ }^{70}$ Evidence is also provided that the implied discount rates increase dramatically ( 0.24 percentage points per day) over the food stamp month.

${ }^{71}$ These effects may be reinforced by cue-driven behavior (as in the models of Laibson, 2001 or Bernheim and Rangel, 2004 discussed above) since the majority of drug users in Vancouver live in close proximity of each other. ${ }^{72}$ Also, the SSI/DI impact on alcohol-related hospital admissions is smaller than that for other drugs - possibly because acute health problems are less common for alcohol.
} 
0.9 percent in the first week of the month due to large increases in mortality due to substance abuse (13.8 percent) but also because of homicide, suicide, other external causes, motor vehicle accidents and liver disease with mention of alcohol (6.5, 5.3, 4.6, 2.8 and 2.6 percent respectively). ${ }^{73}$ They also uncover evidence of smaller (less than 1.0 percent) but still significant first-of-the month effects for deaths due to respiratory or circulatory disorders, neoplasms and liver disease without mention of alcohol.

Using data from the 1973-2005 Multiple Cause of Death Files, Evans and Moore (forthcoming) confirm that mortality increases by 0.9 percent in the first week of the month, relative to the preceding week. This is led by a 3.0 percent rise in deaths due to substance abuse (versus a 0.8 percent increase in other fatalities) but because substance abuse deaths are relatively rare, the absolute within-month fluctuations in mortality are much greater for those from non-substance related causes. ${ }^{74}$ They also show that first-of-the-month increases in various types of consumption (e.g. food and non-food items, lottery tickets, movie box office receipts, as well as foot traffic at malls, retail and apparel establishments) are greatest for the groups most likely to be liquidity constrained (the less educated, government transfer payment recipients, low income households), and that within-month variation in mortality declines with education. Their overall conclusion is that deaths rise at the beginning of the month because many inherently risky activities increase when liquidity constrained consumers have full wallets.

\section{ii. Macroeconomic Fluctuations and Health Behavior}

Many aspects of health-related behaviors exhibit a counter-cyclical variation. Evidence that mortality is procyclical dates back more than 80 years (Ogburn and Thomas, 1922; Thomas

\footnotetext{
${ }^{73}$ They point out that alcohol or substance abuse seems likely to play an indirect role in many deaths from some of the other sources (e.g. homicide and suicide).

${ }^{74}$ They estimate that 647 extra deaths from substance abuse during the first week of the month, compared with 3636 additional fatalities from other sources.
} 
1927) but it is only in the last 15 years that these patterns, and the mechanisms for them, have begun to be understood. A major empirical innovation has been the use of data containing multiple geographic locations observed at several points in time, allowing the use of panel data methods, in particular the inclusion of location-specific fixed-effects and general time effects. ${ }^{75}$ In a series of papers, Ruhm has provided evidence that, when economic conditions (typically proxied by unemployment rates) weaken, heavy drinking and drunk-driving, smoking, obesity, and physical inactivity decrease and diets improve (Ruhm, 1995, 2000, 2005a; Ruhm and Black, 2002). Two potential mechanisms for these effects have been highlighted. First, income reductions during bad economic times appear to reduce some types of unhealthy consumption (e.g. drinking), as in the discussion of full wallet effects above. Second, some healthy behaviors (e.g. exercise) are time-intensive and work hours are generally procyclical.

Other research examining these health behaviors, using similar techniques, generally also finds that behaviors become healthier in bad times. Evidence that alcohol sales and driving problems decline has been provided by Evans and Graham (1988), Ettner (1997) and Freeman (1999), among others; Dee (2001) uncovered a drop in alcohol use and heavy consumption, but also an increase in binge drinking. ${ }^{76}$ Gruber and Frakes (2006) verify the decline in smoking and Courtemanche (2009) shows that shorter work hours decrease obesity because they are associated with increases in exercise and reduce consumption of fast food and prepared processed foods. Xu and Kaestner (2010) use an instrumental variables approach to show that the lower work hours occurring during economic downturns cut smoking and increase exercise while Edwards (2008) indicates that having more non-work time increases sleeping, socializing

\footnotetext{
${ }^{75}$ Ruhm (2008) provides a detailed discussion of these issues, and a review of the related empirical literature.

${ }^{76}$ However, Arkes (2007) finds that drinking and drug use by teenagers is countercyclical.
} 
and time spent caring for the elderly. On the other hand, Charles and DiCicca (2008) find that obesity and BMI rise for men with low ex ante probabilities of employment. ${ }^{77}$

Interestingly, better health during economic downturns occurs even though screening tests (mammograms, pap smears, and colorectal exams) are less often received (Ruhm, 2000) and doctor visits and hospital episodes decrease (Ruhm, 2003; Xu and Kaestner, 2010). However, there are exceptions. Dehejia and Lleras-Muney (2004) find that pregnant women obtain earlier and more extensive prenatal care when the economy is weak and Ruhm (2007) shows a similar decline in sophisticated treatments for heart disease (e.g. coronary bypass and angioplasty) among senior citizens.

The macroeconomic variations in health behaviors provide one reason why mortality is procyclical. Research using the empirical methods just discussed generally predicts that a one percentage point increase in unemployment reduces total mortality by 0.3 to 0.5 percent, with a similar decline in deaths from coronary heart disease and much larger decreases in deaths from external causes (particularly traffic accidents); conversely, cancer mortality changes little as expected since such fatalities are likely to be less responsive to short-term changes in behaviors. $^{78}$

It is not obvious whether these behavioral changes represent rational or non-rational responses to changing incentives. On the one hand, individuals will optimally substitute labor supply from periods when wages are low to those when they are high, implying that it may be optimal to devote less time to health-enhancing behaviors when the economy is robust, even if doing so increases in the risk of death. On the other hand, Evans and Moore (2009) shows that

\footnotetext{
${ }^{77}$ More complete discussions of this literature, including evidence for countries outside the United States, are provided by Ruhm (2006) or Ruhm (2008).

${ }^{78}$ For more detailed reviews of previous research examining how macroeconomic conditions influence mortality, see Ruhm (2005b, 2006, 2008).
} 
the causes of death with high macroeconomic fluctuations are the same ones that exhibit large within-month variations, presumably due to full wallets effects that are inconsistent with intertemporal optimization.

\section{Economic Consequences of Health Behaviors}

We next discuss the challenges to identifying the causal impact of health behaviors and summarize the substantial body of research examining such consequences for a variety of outcomes including: medical care costs, education, employment, wages and crime.

\section{a. Reasons to (and not to) Conduct Cost of Behavior Studies}

Cost of illness (COI) studies calculate the difference in medical costs between those with and without a specific medical condition, controlling for observable characteristics. However, such analyses can be conducted not just for diseases like cancer and diabetes but also for health behaviors such as alcoholism, smoking, drug abuse and a sedentary lifestyle; we refer to these as Cost of Behavior (COB) studies.

Costs can be divided into direct payments for medical care and indirect costs for which no payments are made - such as productivity losses due to job absenteeism or premature death. Public health advocates use COB studies to lobby for greater expenditures to improve health behaviors. However, such arguments are often circular. For example, some health behaviors have substantial medical resources devoted to them, and thus a high COB, but that does not

necessarily justify still greater spending (see, e.g., Shiell et al., 1987). Because medical expenses arise from the decision to treat the outcomes of unhealthy behaviors, a simplistic (but not necessarily desirable) way to reduce these costs is simply to refuse to treat such consequences. Another limitation is that COB studies tend not to consider marginal effects (of expanding or 
reducing the scale of interventions) but instead assume that unhealthy behaviors can be completely eradicated (Shiell et al., 1987). ${ }^{79}$ Given these shortcomings, it is sometimes argued that health economists should devote their efforts not to COI or COB studies but instead to studying the cost-effectiveness of specific interventions designed to change health behaviors (e.g. Roux and Donaldson, 2004).

There are, nevertheless, several situations where COB studies may be of interest. First, they indicate the amount of medical resources currently devoted to treatment - e.g. how much does the U.S. spend treating alcoholism? Second, they enhance our understanding of disparities across gender, race, or income. Third, they provide help in calculating external costs, which may justify government intervention (Zohrabian and Philipson, 2010; Baumol, 1972). Fourth, they represent one input into cost-effectiveness analyses of candidate interventions, by providing estimates of the value of avoided unhealthy behaviors.

\section{b. Challenges to Identifying the Consequences of Health Behaviors}

It is critically important to accurately estimate the causal effects of health behaviors on outcomes (e.g. medical care costs and wages). However, it is frequently the case that only correlations are estimated, which provide limited information because they reflect three factors: 1) the causal impact of unhealthy behaviors on outcomes; 2) the impact of poor outcomes on unhealthy behaviors (reverse causation); and 3) the influence of omitted variables that affect both unhealthy behaviors and poor outcomes (confounding).

In order to estimate the causal effect of unhealthy behavior on an outcome of interest, the most convincing research design would randomly assign large numbers of otherwise similar individuals into treatment and control groups, with the treatment group then compelled to engage

\footnotetext{
${ }^{79}$ Still another potential difficulty is in distinguishing between annual and lifetime costs. For example, Fang and Gavazza (2007) provide evidence that greater investments in medical care prior to age 65 are associated with reduced medical expenditures after that age, along with commensurate reductions in total lifetime spending.
} 
in unhealthy behaviors. Comparing differences in outcomes between the treatment and control groups would then generate a consistent estimate of the impact of unhealthy behaviors. Fortunately for subjects, and unfortunately for researchers, such randomized experiments are neither ethical nor feasible. As an alternative, economists frequently seek out "natural experiments" that feature exogenous variation in health behavior; i.e. not the result of reverse causation (e.g. poor labor outcomes causing unhealthy behaviors) or confounding (e.g. differences in risk aversion or rate of time preference).

Such natural experiments are often exploited using the method of instrumental variables (IV). However, the instruments (i.e. the natural experiments) must be both powerful and valid. ${ }^{80}$ In terms of power, a rule of thumb is that the F statistic for the null hypothesis that the coefficients on the instruments are jointly equal to zero, in the first stage of two-stage least squares, should be 10 or higher (Stock, Wright, and Yogo, 2002). There is no simple convincing test of instrument validity (see, e.g., French and Popovici, 2011). With multiple instruments, over-identification tests can be conducted ${ }^{81}$ However, such over-identification tests are only reliable when the instruments are both powerful and valid (French and Popovici, 2011; Wooldridge, 2002). ${ }^{82}$ As a result, McCloskey (1998) argues that instruments are ultimately accepted (or rejected) based on rhetoric - whether or not the author has made a convincing logical argument for their validity. French and Popovici (2011) point out that IV studies of the consequences of health behaviors conducted during the 1990s often used intuitive or theoretic

\footnotetext{
${ }^{80}$ Angrist and Krueger (2001) and Angrist and Pischke (2009) summarize theoretical and practical difficulties associated with using instrumental variables to identify causal effects; Auld (2006) and Auld and Grootendorst (2011) examine these challenges in the context of health behaviors.

${ }^{81}$ For instance, the two-state least squares residuals can be regressed on all exogenous variables (the instruments and other regressors); the F statistic testing the hypothesis that the coefficients on the instruments are jointly equal to zero is then computed.

${ }^{82}$ More generally, the requirements of power and validity interact in that the bias resulting from an invalid instrument is greater the weaker it is. With sufficiently weak instruments, bias may be larger in IV than OLS estimates and the "cure can be worse than the disease" (Bound, Jaeger, and Baker, 1995).
} 
arguments in favor of instrument validity, whereas those published in the 2000s papers relied more heavily on statistical evidence.

French and Popovici (2011) summarize 60 studies, published between 1990 and 2009, that use IV methods to measure the impact of using alcohol, illicit drugs, and tobacco on a variety of economic outcomes. They note that economists have used the following variables as instruments for risky behaviors: family history of risky behaviors, religiosity, and state policies affecting access and taxes. However, they note that these instruments are now more widely regarded as potentially invalid because of unobserved heterogeneity and policy endogeneity.

Prices of, or taxes on, addictive substances may be valid instruments for their consumption, but power may be lacking. Other, more recently-used instruments for risky behaviors are described in subsequent sections. As in many fields of economics, the search for powerful and valid instruments is ongoing in this literature.

In considering the impacts of health behaviors, it is important to note that some behaviors (like smoking) are never health enhancing. In contrast, increased calorie consumption can reduce the risk of mortality if one is underweight (Flegal et al., 2005, 2007) and moderate consumption of alcohol may improve cardiovascular health (Cook, 2007). Such nonlinear effects of consumption represent an additional challenge for empirical work measuring the consequences of healthy behaviors.

\section{c. Impacts on Medical Care Costs}

Relatively few studies have used econometric techniques to measure the causal effect of health behaviors on medical care utilization or costs. ${ }^{83}$ In contrast, many investigations have, in the tradition of COI studies, estimated the correlation between behaviors and medical costs. For

\footnotetext{
${ }^{83}$ Other methods, such as propensity score matching, could be used to estimate the causal effect of obesity on medical care costs, but to our knowledge have not yet been used for this purpose.
} 
instance, Dorothy P. Rice, one of the pioneers of COI studies, quickly moved from estimating the direct costs of conditions such as cancer and AIDS to estimating the direct costs of health behaviors such as smoking, alcohol abuse, and drug abuse.

Many studies have examined the medical care costs of smoking; Sloan et al. (2004) identifies at least 165 published between the 1960s and 2002. For example, Rice et al. (1986) calculated that $\$ 14.4$ billion was spent in the U.S. in 1980 treating smoking-related illness. In an unusually detailed and careful analysis, Sloan et al. (2004) calculate that smoking at age 24 is associated with $\$ 3,757$ higher lifetime medical expenditures for women and \$2,617 greater expenditures for men (in year 2000 dollars).

Rice et al. (1991) estimated that the direct medical care costs of alcohol abuse were $\$ 6.8$ billion and those related to drug abuse were $\$ 2.1$ billion in 1985. Cook (2007) review COB studies of alcohol abuse; the most recent of which (Harwood, 2000) calculates that the medical consequences of alcohol abuse (including fetal alcohol syndrome) totaled \$19 billion in 1998. The Office of National Drug Control Policy calculates that $\$ 15.8$ billion was spent on medical treatments and prevention of drug abuse in 2002 (Office of National Drug Control Policy, 2004). French et al. (2000) compared self-reported health service utilization among drug users and nonusers and calculate that chronic drug users and injecting drug users generated \$1,000 per year in excess health services utilization relative to non-drug users. Finkelstein et al. (2009) analyze data from the Medical Expenditure Panel Survey (MEPS) and calculate that, in 2006, obese people (i.e. those with a body mass index of 30 or higher) had medical spending that was $\$ 1,429$ (in 2008 dollars) or $41.5 \%$ higher than that for healthy-weight people (those with a body mass index of 18.5 to 25). They calculate, across all payers, $\$ 85.7$ billion (in 2008 dollars) was spent treating obesity in 2006, which represents $9.1 \%$ of all medical spending that year. To reiterate, 
each of these studies estimates the correlation of health behaviors with medical care costs, not the causal effect.

A smaller number of studies use IV methods to estimate the causal effect of health behaviors on medical care utilization and costs. McGeary and French (2000) use access to drug markets, neighborhood sightings of intoxicated individuals and drug sales as instrument for chronic drug use. ${ }^{84}$ They estimate that chronic drug use raises the probability of an emergency room visit by $30 \%$ for females and 36\% for males. Balsa et al. (2008) examines how alcohol consumption affects health care utilization, instrumenting for alcohol consumption using state alcohol and drug policies and other state characteristics (including, curiously, average precipitation). They are unable to reject the exogeneity of alcohol consumption and thus prefer their non-IV estimates which show that moderate drinking decreasing the likelihood of emergency room visits for both sexes and hospitalizations for women but not men. Cawley and Meyerhoefer (2010) estimate the impact of obesity on medical care costs, using obesity status of a biological child to instrument for weight of the parents. Obesity is found to raise annual medical care costs by $\$ 2,826$ (in 2005 dollars), which is more than four times as large as the corresponding OLS estimate (\$676). They hypothesize that OLS results suffer attenuation bias due to measurement error in self-reported weight.

An important direction for future research is to obtain more comprehensive and reliable estimates of the causal effects on medical costs of health behaviors.

\section{d. Impacts on Education}

The effects of alcohol alcohol consumption on educational outcomes have been frequently studied (see the meta-analysis by Lye and Hirschberg, 2010). For instance, the IV estimates of Renna (2007) suggest that binge drinking decreases the probability of graduating

\footnotetext{
${ }^{84}$ The validity of these instruments is questionable if they are correlated with unobserved socioeconomic status.
} 
high school (by age 19 ) by as much as $5.2 \%$ for women and $14.5 \%$ for men. ${ }^{85}$ Using state policies as instruments for drinking, Chatterji (2006a) finds that high school alcohol use has little effect on educational attainment. Cook and Moore (1993) instrument for alcohol consumption using cross-state variation in minimum legal drinking age and conclude that high school seniors who are frequent drinkers (or frequently drunk) eventually complete 2.2 fewer years of college. Dee and Evans (2003) disagree with using across-state variation in such laws out of concern for unobserved heterogeneity across states. Instead, they estimate two-sample IV models in which teen drinking is instrumented using within-state variation in minimum legal drinking ages, and they conclude that teen drinking has no detectable effect on high school completion, college entrance, or college persistence. Koch and Riber (2001) instrument for age of first alcohol use with sibling age of alcohol initiation, and conclude that delaying the start of drinking has small effects on educational attainment - a one year delay in onset is predicted to raised completed education by roughly one-tenth of a year. On the whole, these findings suggest that heavy alcohol consumption by youths decreases educational attainment, but that moderate use may not have a detectable effect.

IV methods have been less commonly used to estimate the impact of drug use on educational outcomes. Chatterji (2006b) uses state drug policies and middle school characteristics as instruments for use during high school but uncovers no evidence of significant effects on educational attainment. The may be due in part to weak instruments.

Two recent investigations - Fletcher and Lehrer (2009) and Ding et al. (2009) - use genetic markers associated with brain chemistry to instrument for obesity and other health conditions when examining how the former is related to educational outcomes. However, this

\footnotetext{
${ }^{85}$ For women, binge drinking is instrumented using state alcohol taxes and state minimum legal drinking age. Those variables do not strongly predict binge drinking by men, so religiosity and whether a parent was a problem drinker are instead used for men. However, these are of questionable validity as they may directly affect education.
} 
requires the unattractive identifying assumption that genes that affect brain chemistry do not affect educational attainment, except through obesity and other regressors; in fact, the specific genes used as instruments in these papers have been linked to many other conditions that could also affect education, such as alcoholism, schizophrenia, aggression, and violence (see Cawley, Han, and Norton, forthcoming). Notwithstanding this caveat, Fletcher and Lehrer uncover little evidence that being overweight during adolescence influences completed years of schooling; however, Ding et al. conclude that obesity lowers grade point average (GPA) by 0.45 (roughly one standard deviation).

Sabia (2007), when using parental obesity status to instrument the weight of the respondent, finds that a 50 to 60 pound (approximately two standard deviation) weight increase reduces the GPA of white females by 8 to 10 percentile points (e.g. from the median to around the $40^{\text {th }}$ percentile) but with little evidence of effects on the GPA of white males or nonwhites.

\section{e. Impacts on Employment}

The most frequently-studied consequences of health behaviors are labor market impacts such as a lower probability of employment or lower wages. For example, a large literature examines how alcohol consumption affects employment. Mullahy and Sindelar (1996) instrument for problem drinking using state tax beer and cigarette taxes, and per capita ethanol sales. Their estimates suggest that problem drinking is associated with statistically insignificant reductions in the probability of employment. Using the same data and similar specifications but allowing for nonlinear effects, Terza (2002) finds that the reduction in employment associated with problem drinking is statistically significant. In contrast, instrumenting for problem drinking using indicator variables for whether county of residence is wet or dry, Feng et al. (2001) uncover a positive and significant association between problem drinking and male employment, 
and an insignificant for females. Using Finnish data and a large number of instruments, Johansson et al. (2007) conclude that alcohol dependency lowers the probability of employment by $50 \%$ for men and $40 \%$ for women. ${ }^{86}$

Negative effects of drug use on employment have also been fairly widely documented. For instance, DeSimone (2002) instruments drug use with the regional price of cocaine and state decriminalization of marijuana, and concludes that marijuana and cocaine use lower employment probabilities by $15-17 \%$ and $23-32 \%$ respectively. Similarly, French et al. (2001) find that chronic drug use lowers employment probabilities by 9 percentage points for both sexes but that light or casual use has no detectable effect. MacDonald and Pudney (2000) estimate a joint model of occupational attainment, unemployment, and drug use using data of British young adults and past hard drug use and current drug use are both associated with a higher probability of current unemployment.

Studies of the impact of obesity on employment obtain mixed results. Cawley (2000) uses weight of a biological child as an instrument for the weight of the mother and finds no significant impact of weight on female employment. Morris (2007) uses the local area prevalence of obesity as an instrument and concludes that obesity reduces the employment of both males and females. Norton and Han (2008) estimate the effect of obesity on employment, instrument for obesity using a similar set of genetic markers related to brain chemistry as was used by Fletcher and Lehrer (2008) to study the impact of obesity on educational outcomes. Using this IV method, Norton and Han (2008) and find no effect of lagged obesity on the employment of either men or women. Rooth (2009) conducted an audit study in which fictitious job applications were submitted for real job openings. Applications sent with a photo of an

\footnotetext{
${ }^{86}$ The instruments include parental alcohol or mental health problems, religiosity, and respondent diabetes or asthma.
} 
obese male (female) were 6 (8) percentage points less likely to receive a job interview than nonobese counterparts.

\section{f. Impacts on Income, Earnings, and Wages}

Cook (2007) notes that alcohol consumption is generally positively correlated with earnings; he refers to this as the "drinker's bonus” but suspects it is the result of reverse causality or confounding. However, a number of high quality studies suggest that the effect may be causal. Auld (2005) examines how drinking and smoking affect income, assuming that religiosity and alcohol/tobacco prices affect smoking/drinking but not income directly. His estimates suggest that moderate alcohol consumption raises income by 10 percent and heavy drinking raises it by 2 percent (relative to abstaining), while smoking reduces income by $24 \%$. Van Ours (2004) instruments for drinking and smoking using dichotomous indicators of initiation before age 16 and concludes that alcohol use raises men’s wages by $10 \%$ while smoking reduces them by the same amount; neither drinking nor smoking affect female earnings.

Early studies of the NLSY that addressed the endogeneity of drug use found mixed results, including finding positive effects of drug use on wages for at least some groups and/or specifications (e.g. Kaestner, 1991; Register and Williams, 1992; Kaestner 1994). Using data of male workers residing in Amsterdam, and with an identification strategy based on the discrete factor method, van Ours (2007) estimates that marijuana use lowers wages by around 10\% but that cocaine use has no wage effect. ${ }^{87}$

The large recent literature estimating how weight affects wages generally finds an obesity earnings penalty for women (especially white females), with less consistent results for men, including sometimes a wage premium associated with being overweight (e.g. Cawley, 2004;

\footnotetext{
${ }^{87}$ Parental cannabis use and the presence of children are used as instruments. He fails to uncover an effect of cocaine use on wages, possibly due to lack of statistical power.
} 
McLean and Moon, 1980). ${ }^{88}$ Using weight of a biological sibling as an instrument, Cawley (2004) estimates that a two standard deviation (roughly 65 pound) increase in weight is associated with 18\% lower wages for white females; however, the OLS estimates, which Hausman tests imply are preferable, are only half as large. Using parental BMI as an instrument in a non parametric model, Kline and Tobias (2008) uncover obesity wage penalties for British men and women. Conversely, using as instruments for obesity the genes related to brain chemistry mentioned above, Norton and Han (2008) fail to detect an obesity wage penalty for either young adults of either sex. Gregory and Ruhm (2011) employ flexible functional forms to show that the wages of women begin to decline after a BMI of around 23. Since this is well within the "healthy" weight range, they speculate that this may reflect returns to "beauty" (Hamermesh and Biddle, 1994) rather than obesity per se. They also find that male wages decline at higher levels of BMI. European studies using weight of relatives as instruments also find obesity wage penalties for women but less so for males (e.g. Lundborg et al., 2007; Brunello and d'Hombres, 2007; Atella, 2008; Greve, 2008), although the effects of obesity vary across nations. $^{89}$

\section{g. Impacts on Crime}

Substance use may lead to criminal activity, for instance, by increasing aggression or lowering inhibitions; see the discussion of "hot” states in section 3. Related research spans both the health economics and economics of crime literatures and can generally be divided into studies directly relating substance use to crime and those evaluating how substance abuse policies or sin taxes (rather than substance use itself) is associated with criminal behavior.

\footnotetext{
${ }^{88}$ McLean and Moon hypothesize that among mature men, large size may signal power and accomplishment; they label this the "portly banker" effect.

${ }^{89}$ Brunello and d'Hombres find that BMI lowers earnings more in Southern than Northern Europe; Lundborg et al. (2007) find particularly large obesity wage penalties in Central Europe.
} 
French and Maclean (2006) instrument underage drinking by young adults with parental drinking problems and state beer taxes. They find that, among males (females) consuming 12 or more alcoholic drinks in the past year is associated with a 30.9 (2.8) percentage point increase in the probability of vandalizing property, a 28.9 (19.8) percentage-point increase in the probability of stealing, and a 50.1 (57.2) percentage-point increase in the probability of committing any illegal act.

Other studies use indirect measures of substance use. Fryer et al. (2005) find that an index of cocaine prevalence (based on factors arrests, emergency room visits, drug busts and newspaper stories involving cocaine) predicts higher homicide rates among black youths. Grogger and Willis (2000) estimate that the introduction of crack cocaine into a city (based on FBI crime reports) raised urban crime rates by roughly $10 \%$.

Taxes that raise the monetary cost of alcohol or drugs, and policies that increase the time cost of acquiring alcohol or drugs, are generally associated with decreases in crime. Carpenter and Dobkin's (2010) comprehensive review of research on alcohol regulation concludes that higher alcohol taxes and age-based restrictions on alcohol availability reduce crime. Markowitz (2005) finds little evidence that increases in drug or alcohol prices reduce violent victimizations but higher beer taxes do appear to reduce alcohol-related assaults. ${ }^{90}$ Carpenter $(2005,2007)$ shows that zero tolerance drunk driving laws for minors reduced male nuisance crimes such as vandalism, public drunkenness and disorderly conduct by 1-2 percent but have no effect on violent crime. Biderman et al.’s (2009) difference-in-differences estimates indicate that homicides were reduced by $10 \%$ when Sao Paulo Metropolitan Area municipalities adopted laws mandating closing hours for bars and restaurants to restrict alcohol consumption. Dobkin and

\footnotetext{
${ }^{90}$ The data set utilized, the National Crime Victimization Survey, provides information about whether alcohol and drugs were involved in an incident.
} 
Nicosia (2009) find that after the U.S. shut down two plants producing more than half of the precursors to production of methamphetamines, felony arrests for methamphetamines fell by half, but there were no significant reductions in property or violent crime. Weatherburn et al. (2002) find that the heroin “drought” in Australia was accompanied by a sharp increase in robberies and breaking and entering offenses, but these quickly declined.

A general methodological concern is that local crime rates are sometimes used as an instrument for drug and alcohol use (e.g. see the review in French and Popovici, 2011), whereas some of the research described here concludes that crime is a consequence of such use.

\section{Strategies for modifying health behaviors}

The first fundamental theorem of welfare economics states that perfectly functioning free markets are Pareto efficient and that government intervention cannot increase social welfare (see, e.g., Mas-Colell, Whinston and Green, 1995). However, there frequently exist failures in markets for addictive substances or other goods involving unhealthy behaviors. The traditional economic approach is for government intervention to fix these market failures. For example, in the case of a negative externality in consumption A.C. Pigou advocates a tax on consumption equal to the amount of the externality, thus internalizing costs of the externality to the decisionmaker and resulting in socially optimal levels of consumption (see, e.g., Baumol, 1972). Another example is that, if consumers lack relevant information, the government can require that manufacturers provide the missing information, leading to socially optimal decisions.

The economic approach differs from the public health perspective. For example, the economic perspective is that the socially optimal levels of unhealthy behaviors are characterized by marginal social benefits equaling marginal social costs, whereas the public health perspective 
seems to be that the socially optimal prevalence of smoking and obesity is zero. The public health perspective is generally supportive of government action to reduce unhealthy behaviors, whether or not there are market failures. Interestingly, recent work emphasizing timeinconsistent or other non-optimizing economic behavior may partially reconcile differences between these two approaches, suggesting situations where government involvement may raise social welfare even in the absence of market failures.

\section{a. Taxes and Subsidies}

\section{i. Economic Rationale for Taxation}

Unhealthy behaviors such as smoking, excessive drinking and obesity impose substantial external costs. As discussed, Pigovian taxes - that are equal to the amount of negative consumption externalities - can correct these distortions and result in socially optimal levels of consumption by agents who equalize marginal benefit with marginal cost.

Measuring the external costs of unhealthy behaviors will always be difficult because randomized controlled trials are unethical and researchers must rely on observational data, with the result that confounding factors (e.g. rate of time preference, cognitive ability, or mental health) may bias estimates and lead to an overstatement of external costs. On the other hand, causal effects may be understated if the economically disadvantaged are both more likely to engage in unhealthy behaviors and have less access to medical care. One promising direction for future research is to find and exploit natural experiments in which behaviors vary but other determinants of medical costs do not.

Manning et al.’s (1991) comprehensive study of participants in the RAND Health Insurance Experiment examined both the costs (e.g. medical costs, sick leave, early retirement, disability benefits, and lost wage tax revenue) and the "benefits” (e.g. lower payments from 
retirement pensions and long-term care insurance) associated with smoking, drinking alcohol, and a sedentary lifestyle. They conclude that lifetime external costs (in 1986 dollars) amount to $\$ 1,000$ per smoker, $\$ 19,000$ per heavy drinker, and $\$ 1,650$ per sedentary person. Put another way, the external costs amount to 15 cents per pack of cigarettes smoked, 54 cents per excess drink, and 24 cents for each mile not walked.

Sloan et al. (2004) provide an even more comprehensive investigation of the external costs associated with smoking that considers: disability life-years, lifecycle earnings, Social Security and Medicare benefits, as well as spouse morbidity, disability, and mortality. They estimate that the lifetime external cost of smoking (net of cigarette taxes paid) is $\$ 3,829$ (in 2000 dollars) for a female 24-year old smoker and \$8,001 for a corresponding 24-year old male; the latter amounts to $\$ 1.44$ per pack of cigarettes.

Obesity imposes external costs through both public and private health insurance. In 2008, obesity-related illness cost taxpayers $\$ 19.7$ billion through Medicare and $\$ 8$ billion through Medicaid, while private health insurance plans paid \$49 billion in 2008 to treat obesityrelated illnesses (Finkelstein et al., 2009). Using the method of instrumental variables in which respondent weight is instrumented using the weight of a biological relative, Cawley and Meyerhoefer (2010) estimate that the causal impact of obesity on medical care costs for Medicaid recipients is $\$ 3,778$ (2005 dollars), of which $\$ 3,647$ is paid by Medicaid; however, given the relatively small sample, these estimates are not significantly significant.

If consumers have time-inconsistent preferences, the optimal tax should include not only external costs but also the internal costs that consumers impose on themselves. Using such reasoning, Gruber and Köszegi (2001) estimate that cigarette taxes should be raised by an additional dollar per pack, while Sloan et al. (2004) obtain dramatically higher (\$32.78/pack) 
estimates of the internal costs of smoking. Consistent with this, Gruber and Mullainathan (2005) report that smokers in the U.S. and Canada are happier in jurisdictions (states or provinces) with higher cigarettes taxes, as might occur with time-inconsistent preferences - because the government is helping them to smoke less, which would prefer but otherwise could not do on their own.

If the goal of policy is to internalize the externalities associated with smoking, then it is clear that cigarettes should be taxed. However, for other unhealthy behaviors the ideal tax policy is less obvious. For instance, moderate drinking may yield medical benefits, as discussed above, implying that higher alcohol taxes could have negative effects on health for moderate consumers. The situation is even more complex for obesity. The most direct application of the Pigovian logic would be to tax body fat; for example, charging the obese a higher premium for their public and private health insurance. However, this may not be politically feasible and would raise concerns about fairness for those with a strong genetic predisposition to obesity. A decision to instead tax the behaviors, goods, or services that contribute to obesity leads to the different problem of determining the ideal scope and structure of such taxes. For example, tax policy is hindered by the impossibility of dividing foods into those that are "bad" (promote obesity) or “good” (prevent obesity). Recently, some public health researchers have called for taxes on fullcalorie soft drinks (e.g. Brownell et al., 2009; Brownell and Frieden, 2009). However, the approach of taxing or subsidizing specific foods raises many questions. For example: should fruit juices that are high in calories also be taxed? Does the answer depend on the vitamin content of the juice? Should diet soft drinks that are low in calories be subsidized? Or should diet soft drinks be taxed because they may promote the habit of consuming sweets or may be complements with other energy-dense foods (Brownell et al., 2009)? Obesity is the result of 
energy imbalance; specifically, more calories consumed than burned or excreted. Thus, obesity can equally well be attributed to insufficient physical activity as to excessive caloric intake. Does this imply that taxes should be increased on complements to sedentary behaviors, such as televisions, video games, or internet access?

Optimal taxation becomes still more complicated still when allowing for cognitive limitations, bounded rationality or other sources of decision errors. For instance, Bernheim and Rangel (2004) point out that many unhealthy behaviors represent mistakes (that have a stochastic component), implying that higher taxes may unfairly penalize people whose genetic endowment predisposed them to drug addiction or other undesirable outcomes. On the other hand, such individuals may most desire government policies as precommitment devices (Gruber and Mullainathan, 2005).

In contrast to the complexities of taxing unhealthy behaviors, the economic argument for eliminating subsidies that promote unhealthy behaviors seems quite strong. For example, current U.S. agricultural policies expand the availability and reduce the cost of "program" crops - like soybeans and corn - that that have become major inputs into processed energy-dense food products (Wallinga et al. 2009; Cawley \& Kirwan, 2011). Removing such subsidies would raise the relative prices of such foods, with the desirable effect of reducing body weight; although, depending on the elasticity of consumption to the price of agricultural commodities, this effect may be small (Cawley and Kirwan, 2011).

\section{ii. Concerns about Regressivity of Taxes}

Vertical equity suggests that those with greater ability to pay should be taxed more heavily than those with less ability to pay (Rosen, 2002). Because those of low socioeconomic status tend to be more likely to engage in many unhealthy behaviors including tobacco use, 
physical inactivity, and poor diet (see, e.g., Pampel, Krueger, and Denney, 2010, and Cutler and Lleras-Muney, 2010), Pigovian taxes on products like cigarettes and energy-dense foods will tend to be regressive. Thus there may be a tradeoff between the two socially desirable goals of vertical equity and population health. In principle, this tradeoff can be avoided by pairing the excise tax with a means-tested income transfer, with the combined effect of allowing the substitution effect of the price change to affect behavior while eliminating the income effect of the price change that would lower utility (see, e.g., Perloff, 2008). In practice this may be difficult to implement. Moreover, it is important to note that taxes imposed on behaviors disproportionately engaged in by the poor will not automatically be regressive, because elasticity of demand for the taxed product may decline with income. However, this also raises the question of whether an attempt should be made to design taxes to focus on those whose (unhealthy) behaviors are most price elastic.

\section{iii. Extent to which Tax Is Passed Through to Retail Price}

In perfectly competitive markets, the pass-through of a tax to retail prices is $\frac{S}{S-D}$, where $S$ and D are the price elasticities of supply and demand (see, e.g., Perloff, 2008). Thus, the passthrough of a tax is bounded by zero and one. However, with imperfectly competitive markets, taxes may be "overshifted" such that prices can rise by more than the amount of the tax (Besley and Rosen, 1999). The logic is that manufacturers may use the tax hike as an opportunity to raise prices, although this begs the question of why the apparently collusive oligopolists were not previously maximizing profits. One possibility is that concerns about fairness constrain profitseeking (Kahneman et al., 1986). For instance, price increases with no apparent justification may cause an outcry among consumers that cuts sales and profits; tax increases then provide manufacturers with an excuse to raise prices. 
Studies confirm that tax pass-through rates sometimes exceed 100\%. For sales tax rates ranging from $0 \%$ to $8.25 \%$, the tax pass-through rate has been estimated to be $100 \%$ for fast food hamburgers and over 100\% for Coca-Cola (Besley and Rosen, 1999). Chaloupka and Warner (2000) review that the pass-through exceeds 100\% for cigarettes, while Young and BielinskaKwapisz (2002) and Kenkel (2005) found pass-through of greater than 100\% when alcohol taxes were increased.

\section{iv. Cross-border shopping, smuggling, and excise tax evasion}

When excise taxes are higher in one jurisdiction than another, there is an incentive for organized or casual cross-border shopping (more pejoratively called 'smuggling'). Beatty et al. (2009) present evidence suggesting that differentials across international borders in rates of alcohol and tobacco taxation result in economically important amounts of cross border shopping and tax avoidance behavior. Chaloupka and Warner (2000) conclude that a large proportion of cigarette sales in states with cigarette taxes represent smuggling to higher-tax states and describe how cross-border shopping between the U.S. and Canada has varied with differences in their tobacco taxes. Tosun and Skidmore (2007) show that when West Virginia raised its food sales tax from $0 \%$ to $6 \%, 1990$, food sales in border counties fell by eight percent, as consumers increasingly made purchases in neighboring states that taxed food at a lower rate or exempted it from sales taxation altogether.

The extent of these effects will generally vary depending on how close customers are to jurisdictional borders. For example, most Texas residents will find cross-border shopping to be uneconomical because of the time and travel costs involved. Conversely, the District of Columbia is so small that it is relatively easy and cheap for residents to shop across the border. Thus, it is estimated that food demand in D.C. is highly elastic to local taxes, with each one 
percentage point increase in the food sales in the District of Columbia, relative to neighboring states, reducing food purchases in the District by 7\% (Fisher 1980).

The implication of this literature is that individual cities and states whose citizens live close to state borders may find that excise taxes reduce domestic sales but have little impact on consumption. If states do not take into account the impact of their lower excise taxes on the health behaviors of residents of neighboring states, there may be a "race to the bottom" in which states jockey to have excise taxes slightly lower than their neighbors in order to increase tax revenue. Excise taxes set at the national level would prevent the race to the bottom, but at the cost of preventing states from tailoring such tax policy to their circumstances.

\section{v. Effect of taxes on unhealthy behaviors}

Section 2 provides detailed information on price elasticities of demand for unhealthy behaviors. The estimates presented there confirm that consumption of even addictive goods is responsive to price, which implies that tax policy can be used to reduce the prevalence of unhealthy behaviors.

Other research explicitly examines the effects of taxes in particular (rather than variation in prices from other sources) on health behaviors. For instance, an analysis of almost 30 years of state-level data found that a $1 \%$ increase in beer taxes is associated with a $1.0 \%$ decrease in youth drinking (Carpenter et al., 2007). Others estimate that a one-dollar increase in the beer excise tax would reduce the prevalence of youth alcohol use by two percentage points, but with no effect on binge consumption (Cook and Moore, 2001). Forster and Jones (2001) use duration analysis to study the decisions to initiate and quit smoking; they estimate that a $5 \%$ increase in the cigarette tax would reduce by $2 \%$ to $3.5 \%$ the total number of years spent smoking. The 1998 Master Settlement Agreement between cigarette manufacturers and the state attorneys 
general immediately increased cigarette prices by 43.5 cents per pack (nearly 20\%) and raised prices further during the next two years. This reduced smoking rates by 13 percent for youths and 5 percent among adults (Sloan et al., 2004) but smoking by pregnant women fell by less than 3\% (Levy and Meara, 2006).

Recent research suggests that food taxes may not have much impact on caloric intake or obesity. For example, existing soft drink taxes have no detectable effect on child weight (Fletcher, Frisvold and Tefft, 2010). This may be due to the fact that existing taxes on soft drinks are quite small - they average $2.7 \%$ of soft drink prices (Fletcher et al., 2010). Larger taxes have been proposed and might have bigger impacts on consumer behavior, but convincing evidence of this has not yet been obtained. For instance, Chouinard et al. (2007) estimate that even a $10 \%$ tax on ad valorem tax on fat in dairy products (milk, cream, cheese, butter, ice cream, and yogurt) would reduce average fat consumption by less than a percentage point. Based on their review of the literature, Powell and Chaloupka (2009) conclude that small taxes and subsidies are not likely to significantly reduce obesity or BMI, but nontrivial price hikes might have a measurable effect.

Consumers may respond strategically to tax increases. For example, smokers respond to higher cigarette prices by switching to cigarettes that are higher in tar and nicotine per cigarette (Farrelly et al., 2004). In addition, smokers respond to increases in cigarette taxes by extracting more nicotine per cigarette (Adda and Cornaglia, 2006). For most age groups, this compensating behavior is so large that the average daily tar intake is unaffected by cigarette taxes, with one study documenting increases in tar and nicotine consumption for 18-24 year olds (Evans and Farrelly, 1998). 
More generally, consumer responsiveness may depend on the salience of the tax (Chetty, Looney, and Kroft, 2009). For instance, taxes that are added at the register may have less effect on purchases than those included in product list prices and thus are seen when consumers make decisions of what to buy. These issues are particularly relevant for efforts to impose Pigovian taxes designed to produce socially optimal levels of consumption.

Another empirical challenge is that variation across jurisdictions in taxes may be correlated with variation across jurisdictions in voter sentiments regarding unhealthy behaviors (DeCicca et al., 2002). For example, cigarette taxes are low in Kentucky but high in California. This may partly occur because Kentucky is a major tobacco-growing state, and California is not; it may also reflect differences across states in preferences about health.

Failure to control for policy endogeneity may bias coefficient estimates of the impact of taxes on health behaviors. For example, DeCicca, Kenkel, Mathios, Shin and Lim (2008) measure state anti-smoking sentiment using data from the Tobacco Use Supplements of the Current Population Survey, which ask respondents their opinions on anti-smoking policies such as clean indoor air laws and restrictions on tobacco promotion and advertising. They then show that controlling for state anti-smoking sentiment leads to a reduction in the estimated price elasticity of demand for cigarettes and that the failure to do so leads to overestimates of the price responsiveness of youth smoking.

\section{vi. Effect of taxes on outcomes subsequent to consumption}

A large literature estimates the effect of alcohol taxes on outcomes subsequent to consumption. (Parallel literatures do not exist for smoking or eating because those behaviors are not contributors to crime, and a related literature does not exist for drugs because illicit drugs are not taxed.) Many of the studies on the effects of alcohol taxes estimate reduced-form models 
using state level data, so the results need to be interpreted with caution, given concerns about policy endogeneity, modest within-state variation in taxes, and omitted variables.

Alcohol taxes (most commonly beer taxes) are negatively correlated with physical child abuse committed by women but not men (Markowitz and Grossman, 2000), child homicides (Sen, 2006), teen abortions (Sen, 2003), gonorrhea and syphilis (Chesson et al., 2000), work days lost due to industrial injuries (Obstfeldt and Morrisey, 1997) and male but not female suicides (Markowitz et al., 2003). Pacula (1998) shows that beer and marijuana are complements, with the result that higher beer taxes reduce marijuana use.

The effects of alcohol taxes on motor vehicle fatalities has been widely studied with most research suggesting a strong negative association (e.g. Cook, 1981; Chaloupka et al., 1993; Ruhm, 1996; Young and Bielinska-Kwapsiz, 2006). Some (but likely not all) of this inverse relationship probably is probably spurious or reflects policy endogeneity (Dee, 1999; Mast et al., 1999; Young and Likens, 2000). Cook and Moore (1993) show that higher alcohol taxes are associated with increased educational attainment but Dave and Kaestner (2002) find little association between alcohol taxes and labor market outcomes. Cook, Ostermann and Sloan (2005) estimate that a permanent reduction of $1 \%$ in alcohol consumption (whether through taxes or another policy) would have a negligible effect on the death rate of those aged 35-69 years but Cook and Tauchen (1982) show that higher alcohol taxes reduce cirrhosis mortality, presumably due to decreases in heavy drinking.

\section{b. Cash incentives for healthy behaviors:}

The TORA predicts that individuals engage in unhealthy behaviors when the discounted lifetime benefits exceed the associated costs. However, other models emphasize the likelihood of mistakes. In these cases (and even with rational addiction if there is ex ante uncertainty about 
outcomes) agents will often attempt to change their unhealthy behavior but fail to do so. Indeed, this is why Orphanides and Zervos (1995), Berheim and Rangel (2004), among others, suggest that harm reduction policies such as subsidized rehabilitation may be desirable.

One possible way to "help people help themselves" is to provide financial incentives for reductions in drinking, drug use, weight, or food consumption, or for increases in physical activity. $^{91}$ Such incentives are potentially useful for several reasons. First, the benefits of behavior change may otherwise not be salient, because their magnitude is not known with any degree of certainty. Second, the benefits of behavior change may not be immediate. (In contrast, the costs of behavior change, such as withdrawal, usually are immediate.) For this reason, the effectiveness of rewards generally declines the further in the future that they occur (Ainslie, 1975). Third, time inconsistent preferences may result in preference reversals and an inability to adhere to plans for more healthful behavior.

Offering immediate cash rewards for behavior change may help to solve these problems of salience, immediacy, and time-inconsistency. Interestingly, even small incentives may be effective if they are salient and provided in exchange for clearly defined short-term objectives. Even small rewards may be effective because people tend not to compare payoffs to their income or wealth but instead "bracket” them - i.e. consider them in isolation (Read et al. 1999; Kahneman and Tversky 1979). Financial rewards can also be structured to create precommitment devices, helping to reduce problems created by time-inconsistent preferences. For example, recovering addicts might post a bond that is automatically forfeited if they relapse.

Contingency management offers incentives for addicts to remain abstinent by providing them with vouchers that can be exchanged for market goods in exchange for negative results on

\footnotetext{
${ }^{91}$ Financial incentives can also be used to encourage patients to receive screening tests, show up for appointments or adhere to recommended regimes for taking prescription medications. In the review by Giuffrida and Torgerson (1997), 10 of 11 studies found that financial incentives improve patient compliance on these outcomes.
} 
drug tests (Higgins et al., 2002). ${ }^{92}$ This program was originally devised for cocaine addicts but has since been applied to the treatment of addiction to alcohol, marijuana, nicotine, and opiates. A meta-analysis of voucher-based reinforcement therapy found overwhelming evidence of increased abstinence; the vouchers raised compliance by an average of 30\%, with larger effect sizes for rewards that were more valuable or were delivered immediately (Lussier et al., 2006). However, contingency management appears to be more effective at treating use of opiates and cocaine than tobacco (Prendergast et al., 2006). A striking feature of these programs is the relatively high success rates obtained for small vouchers - as little as $\$ 2.50$ for a single negative test for cocaine (Higgins et al., 2002) or an $\$ 137$ average payment over a three-month period (Petty and Martin, 2002).

The results for weight loss efforts are more mixed. Cawley and Price (2011) find that worksite programs offering modest cash rewards for specific reductions in weight (e.g. \$30 per quarter for a $10 \%$ weight reduction) were not successful - the treatment group lost slightly less weight over a 12-month period than the control group - although modest improvements were obtained when the treatment group posted substantial (\$110) bonds that were only refunded upon successful achievement of year-end weight loss goals.$^{93}$ Similarly, Finkelstein et al. (2007) present evidence of modest weight loss at three months but no difference at six months for financial rewards ranging from $\$ 7$ to $\$ 14$ per percentage point of weight reduction (after six months), and Butsch et al. (2007) fail to detect significant effects at 12 weeks for a treatment group offered a $\$ 150$ refund of their enrollment fee if they lost $6 \%$ of their initial weight.

Burger and Lynham (2010) examine data from the British bookmaker William Hill of 51 people who placed bets that they would be able to lose a specific number of pounds (verified by

\footnotetext{
${ }^{92}$ Vouchers are awarded instead of cash because recovering addicts might be tempted to spend cash on drugs.

${ }^{93}$ However, attrition was extremely high in this study, $51.2 \%$ after one quarter and $76.4 \%$ after one year.
} 
a physician) over a period of time. Weight loss averaged 78 pounds (from a starting mean of 263 pounds) over an average of 243 days. However, despite payoffs averaging \$2,332, roughly $80 \%$ of bettors failed to meet their weight loss goals. This is an interesting example of the private market offering precommitment mechanisms for time-inconsistent consumers and it is possible that many individuals considered themselves better off for having participated because they lost weight, even if they "lost” their bet.

Gine, Karlan, and Zinman (2010) implemented a voluntary precommitment program for smoking cessation whereby smokers deposited funds for six months and had these deposits returned to them if they tested negative for nicotine use, with the funds forfeited to charity for positive urine test. Those who participated were three percentage points more likely than a control group to have quit smoking at six months, and this effect persisted at 12 months.

The success of such interventions may often be highly dependent on the precise structure of incentives. For example, Volpp et al. (2008) uncovered substantial short-run weight loss (at 16 weeks) for a program in which participants successfully meeting weight loss goals were entered into a lottery with a one-in-five chance of receiving a small (\$10) reward and a one-inone hundred probability of obtaining a large $(\$ 100)$ reward. The short-term weight loss was dramatic (weight reductions averaging 16 pounds), but the longer-term effects (at 7 months) were less so. Similarly, Volpp et al. (2006) found that modest financial incentives combined with enrollment in a smoking cessation program substantially reduced tobacco use in the short term (at 30 and 75 days), but not in the longer run (at 6 months). On the other hand, in a followup intervention, Volpp et al. (2009) found evidence of decreased smoking even at 18 months, 
possibly because the incentives were of relatively large size and dependent on longer-term behavioral changes. ${ }^{94}$

\section{c. Restrictions on Purchase or Use}

A variety of polices have been implemented with the goal of directly restricting availability or raising the time costs for using unhealthy products. A large literature has examined the impacts of state minimum legal drinking ages (MLDA), which make it more difficult for teens to acquire alcohol. Wagenaar and Toomey (2002) conducted an in-depth review of research conducted between 1960 and 2000, covering periods of both falling (during the 1970s) and then rising (during the 1980s) drinking ages. They concluded that the evidence persuasively indicates an inverse relationship between the MLDA and youth alcohol consumption, traffic crashes, and other social problems (like suicides, homicides and vandalism). However, they also find that the evidence is insufficient to say whether the effects vary across subpopulations, such as for college students. More recent analyses confirm these effects. For instance, Cook and Moore (2001) find that youths who are younger than the minimum purchase age for alcohol in their state are 5.5 percentage points less likely to drink in the past 30 days and are 2.5 percentage points less likely to binge drink. A recent analysis of almost 30 years of statelevel data concluded that increases in the MLDA during the 1970s and 1980s reduced drinking participation and heavy drinking by 4\% among high school seniors (Carpenter et al., 2007). However, minimum purchase ages may have the unintended consequence of leading youths to switch from alcohol to drugs: DiNardo and Lemieux (2001) estimate that raising the state MLDA

\footnotetext{
${ }^{94}$ Individuals were paid $\$ 250$ for not smoking 3 or 6 months after program completion and an additional $\$ 400$ if still abstinent at 9 or 12 months. However, reflecting the difficulty of making permanent behavioral changes, only 9.4 percent of the treatment group abstained from smoking at 9 or 12 months (compared to 3.6 percent of the controls).
} 
drinking age from 18 to 21 increases the prevalence of youth marijuana consumption by 2.4 percentage points.

Laws barring youth possession, use, and/or purchase of tobacco also deter smoking participation by teens but with little evidence of changes at the intensive margin for adolescent and young adult smokers (Tauras, Markowitz, and Cawley, 2005).

A large body of research suggests that restrictions on smoking in public places and private workplaces (e.g. clean indoor air laws) reduce the prevalence of tobacco use (see Chaloupka and Warner, 2000, for an extensive review). Some evidence (see Picone, Sloan, and Trogdon, 2004) suggests that bans on smoking in public places also reduce alcohol consumption by women (but not men), which is consistent with complementarity between smoking and drinking. Bitler et al. (2010) show that the impact of clean indoor air laws varies by industry, with larger reductions in smoking among bartenders than for those employed in other industries (e.g. schools, restaurants, and government).

Most related studies are unable to examine the extent to which smoking was displaced from public places to private places such as homes, or the related issue of whether such laws affect the exposure of nonsmokers to environmental tobacco smoke (ETS) in public places or private homes. Two investigations of these issues yield somewhat conflicting results. Adda and Cornaglia (2010) suggest that smoking bans in the U.S. displaced smoking from public to private places, with the net result of increased exposure to ETS by nonsmokers, particularly those sharing a household with smokers. However, Carpenter, Postolek, and Warman (2011) find that Canadian bans on public smoking led to large reductions in ETS exposure in public places for both smokers and non-smokers, and that these laws did not significantly affect ETS exposure in homes. However, they do estimate that non-smokers' exposure to ETS increased at building 
entrances. Neither Adda and Cornaglia (2010) nor Carpenter, Postolek and Warman (2011) find a significant impact of smoking bans on the probability of smoking.

A potential limitation of all of this research is that estimates may be biased by policy endogeneity. Gallet et al. (2006) finds that the adoption of clean indoor air laws is correlated with state characteristics such as political affiliation, urban population, per capita income, and tobacco production.

A dramatic policy enacted to restrict the consumption of an addictive substance was Prohibition, which outlawed the sale and purchase (but not use) of alcohol in the United States from 1919 to 1933. Individual-level data on alcohol consumption do not exist for this era, but the impact of Prohibition on heavy alcohol consumption has been estimated using deaths from cirrhosis of the liver or alcoholism (Miron and Zwiebel, 1991) and police records regarding arrests for drunkenness (Dills, Jacobson, and Miron, 2005). Both proxy measures suggest that alcohol consumption initially fell sharply, to around 30\% of the previous level, immediately after the enactment of Prohibition, before rebounding over the next several years to between 60 and 70 percent of its prior level (Miron and Zwiebel, 1991). These proxy measures also suggest that, after the repeal of Prohibition, heavy drinking did not initially change but returned to the preProhibition level after a decade.

Drug legalization may be analogous to the repeal of Prohibition. Miron (2003) estimates that the black market prices of cocaine are 2 to 4 times higher than they would be if the drug was legal and that heroin prices are 6 to 19 times as high. Legalization would therefore decrease prices substantially, resulting in higher consumption (see section 2 of this chapter for estimates of the price elasticity of demand for illicit drugs). 
Consumption of illicit drugs is deterred not only by higher prices but also by the legal penalties for purchase and possession. For example, fines for possession and the probability of arrest decrease marijuana use among young adults (Farrelly et al., 2001). However, doubling the fines for marijuana possession would reduce the probability of use by youths by less than $1 \%$, while decriminalization would in increase it by 4 to 5\% (Chaloupka, Grossman, and Tauras, 1999; Saffer and Chaloupka, 1999). On the other hand, doubling of the fines for cocaine possession would reduce corresponding use by roughly $4 \%$ (Chaloupka, Grossman, and Tauras, 1999).

Some studies have examined the impact of increased drug law enforcement on drug price, purity, and consumption. For example, Weatherburn et al. (2002) investigate the Australian heroin "drought”, in 2000, that partly resulted from increased law enforcement. Using a survey of 165 heroin users in that country's largest heroin market, they found that the drought raised prices and lowered purity of heroin, reduced consumption and rates of overdose, but that the associated health benefits were partially offset by increased use of other drugs, most commonly cocaine. Dobkin and Nicosia (2009) examine the impact of the U.S. government's decision in 1995 to shut down two suppliers providing more than half of the precursors used to produce methamphetamine. Focusing on California, they authors find that the supply of methamphetamine was halved, purity declined from 90 to 20 percent, and the price tripled. Use of the drug among arrestees declined 55 percent, and related hospital admissions fell 50 percent. However, the impact was largely temporary, with the price restored to its original level within four months and other outcomes returned to their original levels within 18 months (suggesting that meth producers were able to find substitute ingredients). In contrast to Weatherburn et al. 
(2002), Dobkin and Nicosia (2009) find little evidence of substitution away from the newly expensive drug and towards other drugs.

An empirical challenge to measuring the causal effect of policies on health behaviors is that the policies are endogenous, and are more likely to be adopted in states where voter sentiment is against such unhealthy behaviors. For example, Cawley and Liu (2008) find that state laws to prevent or reduce childhood obesity (such as mandatory physical education for school-aged children) are more likely to be enacted in states with large gaps between the desired and actual weight of adults. Carpenter et al. (2007) finds that alcohol consumption fell just as much 1-2 years before as 1-2 years after a rise in the minimum legal drinking age; they interpret this as evidence that increases in the minimum legal drinking age are endogenous responses to high levels of teen drinking (they find no evidence that zero tolerance underage drunk driving laws are endogenously adopted).

For this reason, simple estimates of the impact of policies on health behaviors may suffer from omitted variables bias. Ruhm (1996) uses state fixed effects to control for differences across states in (e.g.) unobserved social attitudes against drinking and finds that omitted variables strongly affect parameter estimates for policies designed to deter drunk driving (but not alcohol taxes).

\section{d. Providing information:}

Information is generally a public good, and as a result is underprovided by private markets (see, e.g., Perloff, 2008). When consumers have incomplete information, free markets may fail to maximize social welfare (Mas-Collel et al., 1995), providing an efficiency rationale for the government to either deliver the missing information or to require suppliers to do so. Orphanides and Zervos (1995) discuss how information, education, or counter-advertisement 
efforts may be desirable, even with perfectly rationality, to reduce ex ante errors in subjective probabilities - particularly because individuals tend to underestimate their own probabilities of becoming addicts, in part because they overstate rates of substance use by peers. Information that reduces the divergence between subjective and objective risk assessments may therefore improve ex post utility.

Consumers sometimes respond strongly to the provision of new information. A dramatic example is the release of the first Surgeon General's report on smoking and health in 1964, which was followed by an immediate 5\% decrease in smoking; other research indicates that both warning labels on cigarette packs and paid anti-smoking advertisements significantly cut tobacco use (Chaloupka and Warner, 2000). However, the reductions were larger for more highly educated individuals (Grossman, 2001), perhaps reflecting differences in cognitive ability. Information about adverse health consequences can also decrease the use of other addictive goods. For instance, perceived risk of harm from regular use is negatively with the probability of smoking marijuana in the past year (Pacula, 2001).

These findings are not limited to addictive products. In an experiment in Kenya, teenagers in randomly selected schools were provided information that the HIV infection was more common among adult males than teenage boys. This information led to a 61 percent reduction in the impregnation of teenage girls by adult males, as girls substituted away from unprotected sex with older men toward condom-utilizing sex with teenage boys (Dupas, 2011). Information campaigns to prevent HIV/AIDS in Africa have the largest impact for better educated persons (DeWalque, 2007).

Consumers also respond to nutritional information. The Nutrition Labeling and Education Act (NLEA) required manufacturers of packaged foods to provide information about 
their products in the form of the Nutrition Facts panel. One study concludes that this increased the consumption of iron and fiber, without affecting affect consumption of total or saturated fat or cholesterol (Variyam 2008). However, other research suggests that the Nutrition Facts panel led more consumers to choose low-fat options (Mathios, 2000) and that the NLEA lowered obesity among white females by 2.4 percentage points (Variyam and Cawley, 2006). Notably, competition between food manufacturers did not result in this information being provided prior to the government mandate (Mathios, 2000). Between 1975 and 1985, government campaigns to encourage lower consumption of fats successfully reduced the fat intake of U.S. women. Even larger decreases were observed after 1985, when food companies received permission to make health claims about their products (Ippolito and Mathios, 1995). Most discussion of policies to counter externalities involves an increased role for government, but this is an example of how in certain instances, decreasing regulation can reduce market failures, improve efficiency, and enhance social welfare.

One recent policy innovation, implemented in New York City in 2008 and as part of the U.S. health care reform bill in 2010, requires calorie labeling on menus and menu boards in restaurant chains. Elbel et al. (2009) found that the New York City labeling law raised the percentage of customers who reported seeing calorie labels at four major fast-food chains (relative to controls in Newark, NJ which does not have a labeling law); however, calories, saturated fat, sodium, or sugar in the food actually purchased did not change. On the other hand, Bollinger et al. (2010) found that the New York City law reduced calories per transaction at Starbucks by 6\% (15 calories), almost entirely due to decreases in calories from food (rather than beverages). ${ }^{95}$ Wisdom et al. (2010) summarize the experimental data showing that customers

\footnotetext{
${ }^{95}$ They utilize rich data on every transaction at Starbucks stores in New York City and the control cities Boston and Philadelphia (with no calorie posting), from January 1, 2008 until February 28, 2009 - before and after the law's
} 
provided calorie information at a fast-food restaurant ordered meals with around 60 fewer calories than those not receiving the information.

\section{e. Advertising Restrictions}

A common public health response to a high prevalence of unhealthy behaviors involving legal substances such as tobacco, alcohol, or energy-dense foods is to ban or regulate advertisements for these products, or to call for voluntary limits on advertising by manufacturers. (For a description of the history of U.S. regulation of cigarette advertising, see Nelson, 2006.)

Saffer and Chaloupka (2000) examine the impact of various bans on cigarette advertising in 22 OECD countries during the period 1970-1992. They conclude that comprehensive bans (i.e. bans on such ads on television, radio, print, outdoors, movies, sponsorship and at point of purchase) can reduce tobacco consumption but that more limited restrictions have little or no effect. However, the results of later research are more ambiguous. Blecher (2008) extends the approach of Saffer and Chaloupka (2000) to 30 developing countries over the period 1990-2005 and finds that both comprehensive and limited policies to restrict tobacco advertising reduce smoking. Nelson (2003) examines data for 20 OECD countries over the period 1970-1995 and concludes that Saffer and Chaloupka's (2000) results are not robust to the use of stationary data in the form of consumption growth rates, or to controlling for other policies (such as warning labels), or analyzing different time periods; Nelson (2003) concludes that advertising bans, whether comprehensive or limited, do not affect cigarette consumption, which is also the conclusion of meta-analysis of 9 studies examining the U.S. government's 1971 ban on television broadcast advertising of cigarettes (Nelson, 2006).

April 1, 2008 implementation. Most of the reduction in calories was due to consumers buying fewer food items, rather than substituting to lower calorie foods. 
While television advertisements of cigarettes have been illegal in the U.S. since 1971, television advertisements of liquor were kept off the airways by a voluntary agreement among manufacturers until November 1996, when the liquor industry's national trade organization agreed to lift the self-imposed ban (see Frank, 2008),.

Nelson (2010) examines the relationship between advertising bans and alcohol consumptions in 17 OECD countries between 1975 and 2000. He criticizes earlier studies for failing to control for the stringency of other alcohol policies, speculating that this may have led to omitted variables bias in estimates of the impact of the advertising bans. In his preferred models, Nelson (2010) detects no impact of alcohol advertising bans on the demand for alcohol.

Recently, researchers have begun to estimate the possible impact of regulation of advertisements for energy-dense foods. Using data from the NLSY79 and NLSY97, Chou, Rashad, Grossman (2008) estimate that a ban on fast food television advertisements would reduce the prevalence of overweight among 3-11 year olds by 18 percent and the fraction of overweight adolescents (12-18 year olds) by 14 percent. Eliminating the tax deductibility of TV advertising for fast food companies (which the authors state would raise the price of advertising by 54 percent) is estimated to decrease the share of overweight children by 7 percent and of overweight adolescents by 5 percent. However, these estimates may fail to account for the likelihood that advertising may currently be targeted towards heavier youths.

\section{f. Defaults and choice architecture}

Behavioral economists emphasize that individuals frequently make systematic mistakes, raising the possibility that social welfare can be improved by changing the default options to account for factors such as procrastination, lack of self-control and status quo bias. A key component of these interventions, referred to as "libertarian" or "asymmetric" paternalism 
(Loewenstein et al., 2007; Thaler and Sunstein, 2008) is that few limitations are placed the available choice set but small cognitive costs are charged for individuals to select options that are perceived by planners to represent ex post mistakes. A risk of this strategy is that planners may underestimate the knowledge and sophistication of consumers, and may needlessly distort decisionmaking, lowering social welfare.

There are many potential applications of behavioral economics principles to unhealthy behaviors, with food consumption having received the most attention. Examples of proposed policy changes include moving energy-dense items to less convenient locations in school cafeterias, making water rather than soft drinks the default beverage option for fast food meals; or having food choices be made several hours before the meal will be eaten (Just, 2006; Lowenstein et al., 2007).

While such policies hold considerable promise, empirical analyses of them has just begun to be conducted and it will be some time before we have high-quality evaluations of these interventions. To provide one example, Wisdom et al. (2010) find that making lower-calorie sandwiches more salient, by listing them first on the menu, had no effect on total calories consumed because, although these sandwiches were more often ordered, the calorie savings was compensated for by increased consumption of other products. However, a "stronger" intervention, in which individuals had to unseal an envelope with additional menu choices, to purchase more caloric items, did reduce total energy intake.

\section{Future Directions}

Economic research on health behaviors has reached the stage of “early adolescence.” At the beginning of this chapter, we documented the remarkable growth of related economic 
analysis, largely dating from the 1990s. During the "infancy" period, economists demonstrated to skeptical health professionals and policy-makers that economic factors really do play a role in determining health behaviors like drinking and smoking. Much of this research was documented at length in dedicated chapters in volumes 1B of this Handbook (Chaloupka and Warner, 2000; Cook and Moore, 2000; Kenkel, 2000). The early effort was highly successful in convincing public health researchers and practitioners that health behaviors are responsive to prices and other incentives. As a result, taxes are now routinely used by State and Federal governments in their attempts to reduce smoking and alcohol consumption and are receiving considerable attention in current efforts to reduce obesity.

The "toddler" years were dominated by theoretical development and empirical testing of the model of rational addiction. This work has also been extremely influential, particularly among economists, for most of whom the TORA is often the default model for examining health behaviors. This framework has many attractive features, including emphasizing the role of prices and forward-looking behavior, an appreciation of the distinction between long-run and short-run elasticities, and for demonstrating that even seemingly undesirable outcomes can be consistent with fully rational decision-making. With additional assumptions it can explain many interesting phenomenon like quitting “cold turkey”, cycles of binging and purging, and entry into addiction following adverse life events.

That said, the assumptions required for the TORA - including perfect foresight, complete optimization and time-consistent preferences - may be violated for many health behaviors. During the "childhood" of research in this area, economists began to incorporate such considerations into their models of health behavior. Thaler and Sunstein (2009) note that health economists have begun to examine the actual behavior of homo sapiens rather than the stylized 
behavior of "homo economicus". We anticipate that such work will continue during the coming decade or two, the period of "adolescence" in the economic analysis of health behaviors, and we are optimistic that this work will yield important theoretical and empirical advances that lead to improvements in public health policy that enhance social welfare.

This discussion does not imply that there is nothing more to be gained from the traditional theoretical and empirical models. To the contrary, we expect that the majority of economic analyses on health behaviors in the near future will be based on such models, in part because most current health economists have familiarity and expertise with them but also because standard utility-maximization is simple, powerful, and will continue to yield important insights. We therefore conclude this chapter by providing a brief overview of what we see as promising areas for future study first focusing on traditional economic models of health behavior and then moving to less standard frameworks.

\section{a. Future Research Using Traditional Economic Models}

Policy endogeneity represents an important challenge for efforts to estimate the causal impact of policies on health behaviors. Many previous studies take state taxes and substance use policies to be exogenous, but the median voter theorem implies that states with strong voter sentiment against unhealthy behaviors will enact policies designed to discourage them (e.g. DeCicca, Kenkel, Mathios, Shin and Lim, 2008; Cawley and Liu, 2006) and policies may be enacted in response to high levels of substance use (see, e.g., Carpenter et al., 2007). While there is growing appreciation of the problem of policy endogeneity, there is mixed evidence of the extent to which it biases estimates (see, e.g., Carpenter et al., 2007 and Ruhm, 1996), making this an important area for additional research. 
Future empirical investigations will certainly benefit from new data collection, but also from better use of existing data. For instance, there is increased awareness in obesity research that body mass index is a noisy measure of fatness. Recently, economics studies have begun to use alternative measures of fatness, such as percent body fat (which can be calculated using several methods, including bioelectrical impedance analysis) and waist circumference (see, e.g. Burkhauser and Cawley, 2008; Johannson et al., 2009; Wada and Tekin, 2010) but economists are limited by the available data; for instance, many secondary datasets include self-reported weight and height but not more accurate measures of fatness. Biomarkers are also becoming more available in data used by economists, suggesting that such research will become more common in the future. ${ }^{96}$ For example, Adda and Cornaglia (2006) examine the concentration of cotinine, a metabolite of nicotine, in bodily fluids and find that smokers compensate for tax hikes by extracting more nicotine from each cigarette smoked. In subsequent work, Adda and Cornaglia (2010) use data on cotinine concentration to determine whether taxes and public smoking bans affect exposure to environmental tobacco smoke. For an in-depth discussion of the uses and limitations of biomarkers in social science data, see volume 55 (2009), issue 2 of Biodemography and Social Biology.

More in-depth analysis will be facilitated by richer data. For example, recent research on smoking (Loomis et al., 2006), drinking (Bray et al., 2009), and food purchases (Zhang et al., 2008) documents purchases by individual consumers using scanner data from retail establishments, rather than relying on aggregate sales data or recall in consumer surveys. Similarly, heart rate monitors, pedometers, and accelerometers are being used to measure

\footnotetext{
${ }^{96}$ For information on biomarkers in U.S. population-based data see: http://biomarkers.uchicago.edu/studiescollectingbiomarkers.htm.
} 
physical activity (e.g. see Berlin et al., 2006), although each has limitations when applied to general populations (Sirard and Pate, 2001).

However, data limitations continue to pose obstacles for many potentially interesting analyses. For example, it is hard to survey the severely drug addicted because they may not have a permanent residence or phone; as a result, even large social science datasets may not provide statistical power for an analysis of heavy drug users. Another example is that data on food consumption tends to be collected over brief periods (e.g. using 24-hour dietary recalls) in repeated cross sections, longitudinal data would better allow for more in-depth study of the dynamics of eating and weight changes. Information on mental health, and its determinants, is generally not as good as that for physical health and large secondary data sets rarely provide reliable information on job and non-job sources of stress. The field would also benefit from better theoretical definitions of peer and reference groups, and data corresponding to these categorizations.

Perhaps most importantly, most of the empirical evidence summarized in this chapter focuses on a limited set of health behaviors - particularly smoking, drinking, substance use, and obesity. This reflects a relative paucity of research on other outcomes, although some research has been conducted on behaviors like risky sexual activity and prostitution (Oettinger, 1999; Levine, 2001; Gertler et al., 2005), immunizations (Philipson, 1996; Mullahy, 1999) and seatbelt or motorcycle helmet use (Carpenter and Stehr, 2008; Dee, 2009). ${ }^{97}$ Future analyses of a wider array of health behaviors are likely to be highly informative.

\section{b. Future Research Using Nontraditional Models}

\footnotetext{
${ }^{97}$ Chapters on prevention (Kenkel, 2000) and infectious diseases (Philipson, 2000) in volumes $1 \mathrm{~A}$ and $1 \mathrm{~B}$ of this Handbook cover some of these issues in greater detail.
} 
This chapter highlights the promise of emerging non-traditional models that marry strengths of the standard rational economic framework with an understanding of biological considerations, and which incorporate insights from behavioral economics and neuroeconomics. From a modeling perspective, the most important development to date has been the use of quasihyperbolic discounting, which provides a straight-forward method of incorporating time inconsistent preferences into otherwise conventional frameworks. However, this is just one way of capturing such behavior, and it does not account for other aspects of observed decisionmaking, such as the apparent heterogeneity of discount rates across types of purchases or alternative mental states. We anticipate that there will be active research over the coming decades aimed at more realistically modeling decision-making related to health behaviors. We anticipate active research over the next decades to continue this process. Several areas of study seem particularly promising.

Increased attention is being paid to the role of genetic determinants of health, motivated in part by the mapping of the human genome and the inclusion of genetic markers on datasets that are frequently used by economists (e.g. the National Longitudinal Survey of Adolescent Health). To date, most economics research in this area has used genetic markers as instrumental variables when examining how specific behaviors or health conditions affect outcomes such as educational attainment and school performance (Ding et al., 2009; Fletcher and Lehrer, 2009) or labor market outcomes like employment or wages (Norton and Han, 2008). ${ }^{98}$ Thoughtful use of genetic markers in health economics will require awareness of the following issues (see, e.g., Conley, 2009 and Cawley, Han and Norton, forthcoming). First, behaviors are often influenced by multiple genes (they are polygenic) in ways that are difficult to quantify. Second, behavior is

\footnotetext{
${ }^{98}$ Goldman, et al. (2005) provide a detailed discussion of how genetics influence addictive behaviors but do not integrate this into an economic model.
} 
often the result of complicated interactions between genes and environment. Finally, genes tend to affect multiple health behaviors and conditions, implying that genes may be invalid instruments in many contexts.

The emerging field of neuroeconomics also offers promise, some of which may have begun to be realized through the use of brain scans undertaken while individuals engage in the decision-making related to health and other behavioral outcomes (e.g. McClure et al., 2004; Glimcher et al., 2007; Hare et al., 2009). However, it is not yet clear to what extent brain structure is a dominant determinant of economic behavior nor, even if it is, whether current methods of examination provide useful information. Thus, it is difficult to refute Rubinstein's (2008, p. 493) conclusion that brain studies, while “fascinating,” have not yet yielded fundamental insights that change economics.

Additional interdisciplinary work with biomedical researchers is almost certainly desirable, in part because health behaviors are influenced by the system in which medical care is provided. For example, evidence suggests that physician counseling raises the likelihood that sedentary individuals increase physical activity (Calfas et al., 1996) and that tobacco users stop smoking (Stead et al., 2008) but many patients, particularly ethnic minorities, do not receive such advice from their doctors (U.S. Department of Health and Human Services, 2009). A second reason is that while economists are especially well-trained in addressing potential design problems in randomized experimental designs - such as attrition or substitution bias, heterogeneous treatment effects, or treatment contamination - they generally lack a corresponding understanding of biological or medical aspects of the interventions.

Finally, insights from other social sciences - particularly psychology and sociology - are likely to provide rich additions to traditional economic models. Indeed, some of the most 
exciting recent economic research on health behaviors has incorporated factors such as peer groups, social capital and relative status, all of which originally arose from other social science disciplines. In addition, many stylized facts that are central to behavioral economics were first identified by psychologists. One of the great strengths of economics is its ability to incorporate useful theories and findings from other disciplines while retaining a central role for incentives, tradeoffs and constrained optimization. Continuation of this process is likely to allow exciting progress to be made in understanding the determinants of health behaviors and in developing public policies and interventions that can enhance social welfare. 


\section{Works Cited}

Adams, Walter. 1952. "Price Policies in the Cigarette Industry by William H. Nicholls [book review]” American Economic Review, 42(3): 461-463.

Adda, Jérôme and Francesca Cornaglia. 2006. “Taxes, Cigarette Consumption and Smoking Intensity”, American Economic Review 96(4), September, 1013-1028.

Adda, Jérôme and Francesca Cornaglia. 2010. "The Effect of Bans and Taxes on Passive Smoking.” American Economic Journal: Applied Economics. 2(1): 1-32.

Adler, Nancy E., Thomas Boyce, Margaret A. Chesney, Sheldon Cohen, Susan Folkman, Robert L. Kahn, and S. Leonard Syme (1994) "Socioeconomic Status and Health: The Challenge of the Gradient”, American Psychologist, 49(1), January, 15-24.

Ainslie, G. (1975). "Specious Reward: A Behavioral Theory of Impulsiveness and Impulse Control.” Psychological Bulletin. 82(4): 463-496.

Ainslie, George (1991), “Derivation of 'Rational’ Economic Behavior from Hyperbolic Discount Curves”, American Economic Review, 81(2), 334-340.

Akerlof, George (1991), "Procrastination and Obedience”, American Economic Review, 81(2), May, 1-19.

Andreyeva, Tatiana, Michael W. Long, and Kelly D. Brownell. 2010. “The Impact of Food Prices on Consumption: A Systematic Review of Research on the Price Elasticity of Demand for Food.” American Journal of Public Health, 100(2): 216-222.

Angeletos, George-Marios, David Laibson, Andrea Repetto, Jermey Tobacman and Stephen Weinberg (2001), “The Hyperbolic Consumption Model: Calibration, Simulation, and Empirical Evaluation”, Journal of Economic Perspectives, 15(3), Summer, 47-68.

Angrist JD, Krueger AB. 2001. "Instrumental variables and the search for identification: from supply and demand to natural experiments.” Journal of Economic Perspectives 15: 6985.

Angrist JD, Pischke J. 2009. Mostly Harmless Econometrics: An Empiricist’s Companion. Princeton University Press: Princeton, NJ.

Antsey, Kaarin J., Lee-Fay Low, Helen Christensen, and Perminder Sachdev (2009), "Levels of Cognitive Performance as a Correlate and Predictor of Health Behaviors that Protect Against Cognitive Decline Late in Life: The Path Through Life Study”, Intelligence, 37(6), November-December, 600-606.

Argys, Laura M. and Daniel I. Rees. 2008. "Searching for Peer Group Effects: A Test of the Contagion Hypothesis,” Review of Economics and Statistics 90(3): 442-458.

Ariely, Dan and Geroge Loewenstein. 2006. “The Heat of the Moment: The Effect of Sexual Arousal on Sexual Decision Making”, Journal of Behavioral Decision Making, 19(2), April, 87-98.

Ariely, Dan and Klaus Wertenbroch (2002), "Procrastination, Deadlines, and Performance: SelfControl by Precommitment”, Psychological Science, 15(3), May, 219-224.

Arkes, Jeremy (2007), “Does the Economy Affect Teenage Substance Use?”, Health Economics, 16(1), January, 19-36.

Atella, Vincenzo, Noemi Pace, and Daniela Vuri. 2008. “Are employers discriminating with respect to weight? European Evidence using Quantile Regression.” Economics and Human Biology, 6: 305-329.

Auld, M. Christopher. 2005. Smoking, drinking, and income. The Journal of Human Resources 15: 504-518. 
Auld, M. Christopher. 2006. "Using Observational Data to Identify the Causal Effects of HealthRelated Behaviour.” In: Andrew M. Jones (ed.), The Elgar Companion to Health Economics. New York: Edward Elgar.

Auld, M. Christopher and Paul Grootendorst. 2011. "Challenges for Causal Inference in Obesity Research.” In: John Cawley (editor), Handbook of the Social Science of Obesity. New York: Oxford University Press.

Auld, M. Christopher and Paul Grootendorst. 2004. An empirical analysis of milk addiction. Journal of Health Economics, 23: 11117-1133.

Auld, M. Christopher and Lisa M. Powell. 2009. "Economics of Food Energy Density and Adolescent Body Weight.” Economica, 76: 719-740.

Avery, Rosemary, Donald Kenkel, Dean Lillard, and Alan Mathios (2007). "Private Profits and Public Health: Does Advertising Smoking Cessation Products Encourage Smokers to Quit?” Journal of Political Economy 115 (3): 447-481.

Balia, Silvia and Andrew M. Jones (2008), "Mortality, Lifestyle and Socio-economic Status”, Journal of Health Economics, 27(1), January, 1-26.

Balsa AI, Homer JF, Fleming M, French MT. 2008. Alcohol consumption and health among elders. The Gerontologist 48: 622-636.

Beatty, Timothy K. M.; Larsen, Erling Roed; Sommervoll, Dag Einar. 2009. “Driven to Drink: Sin Taxes Near a Border.” Journal of Health Economics, v. 28, iss. 6, pp. 1175-84.

Becker, Gary S. 1976. The Economic Approach to Human Behavior. (Chicago, IL: University of Chicago Press).

Becker, Gary, and Kevin Murphy. 1988. “A Theory of Rational Addiction” Journal of Political Economy, 96(4): 675-700.

Becker, Gary S., and Kevin M. Murphy. 1993. A Simple Theory of Advertising as a Good or Bad. Quarterly Journal of Economics 108:941-64.

Becker, Gary S., Michael Grossman and Kevin M. Murphy. 1994. “An Empirical Analysis of Cigarette Addiction,” American Economic Review, 84(3): 396-418.

Berlin, Jaime E., Kisti L. Storti and Jennifer S. Brach (2006), "Using Activity Monitors to Measure Physical Acticvity in Free-Living Conditions”, Physical Therapy, 86(8), August, 1137-1145.

Bernheim, B. Douglas and Antonio Rangel (2004), “Addiction and Cue-Triggered Decision Processes”, American Economic Review, 94(5), December, 1558-1590.

Berns, Gregory S., David Laibson and George Loewenstein (2007), “Intertemporal Choice Toward an Integrative Framework”, Trends in Cognitive Science, 11(11), November, 482-488.

Besley, Timothy and Rosen, Harvey. 1999. "Sales Taxes and Prices: an Empirical Analysis." National Tax Journal, 52(2): 157-178.

Bhattacharya, Jay, Dana Goldman and Neeraj Sood. 2009. "Market Evidence of Misperceived Mortality Risk”, Journal of Economic Behavior and Organization, 72(1), October, 451462.

Biderman, Ciro, Joao M. P. De Mello, and Alexandre Schneider. 2009. “"Dry Laws and Homicides: Evidence from the Sao Paulo Metropolitan Area,” The Economic Journal, 120: 157-182.

Bitler, Marianne P., Christopher Carpenter, Madeline Zavodny. 2010. "Effects of VenueSpecific State Clean Indoor Air Laws on Smoking-Related Outcomes.” Health Economics, 19(12): 1425-1440. 
Blecher, Evan. 2008. "The impact of tobacco advertising bans on consumption in developing countries.” Journal of Health Economics, 27: 930-942.

Borghans, Lex and Bart H.H. Golsteyn. 2006. Time discounting and the body mass index: Evidence from the Netherlands. Economics \& Human Biology, 4(1): 39-61.

Bray, Jeremy W., Brett R. Loomis, and Mark Engelen (2009), "You Save Money When You Buy in Bulk: Does Volume-Based Pricing Cause People to Buy More Beer”, Health Economics 18(5), May, 607-618.

Brocas, Isabelle and Juan D. Carrillo (2008), “The Brain as a Hierarchical Organization”, American Economic Review, 98(4), September, 1312-1346.

Bound, J., D. Jaeger, and R. Baker (1995). "Problems with instrumental variables estimation when the correlation between the instruments and the endogeneous explanatory variable is weak.” Journal of the American Statistical Association 90 (430), 443-50.

Brownell, Kelly D., Thomas Farley, Walter C. Willett, Barry M. Popkin, Frank J. Chaloupka, Joseph W. Thompson, and David S. Ludwig. 2009. "The Public Health and Economic Benefits of Taxing Sugar-Sweetened Beverages.” New England Journal of Medicine, 361: 1599-1605.

Brownell, Kelly D. and Thomas R. Frieden. 2009. "Ounces of Prevention - the Public Policy Case for Taxes on Sugared Beverages.” New England Journal of Medicine, 360(18): 1805-1808.

Brownson, Ross C., Tegan K. Boehmer, and Douglas A. Luke (2005), "Declining Rates of Physical Activity in the United States: What are the Contributors?” Annual Review of Public Health, 26, April, 421-443.

Brunello, G., D’Hombres, B., 2007. Does body weight affect wages? Evidence from Europe. Economics \& Human Biology 5, 1-19.

Burger, Nicholas and John Lynham. 2010. "Betting on weight loss ... and losing: personal gambles as commitment mechanisms.” Applied Economics Letters, 17(12): 1161 - 1166.

Burkhauser, Richard V. and John Cawley (2008), "Beyond BMI: The Value of More Accurate Measures of Obesity in Social Science Research”, Journal of Health Economics, 27(2), March, 519-529.

Butsch, W.S., Ard, J.D., Allison, D.B., Patki, A., Henson, C.S., Rueger, M.M., Hubbert, K.A., Glandon, G.L. and Heimburger, D.C. (2007). "Effects of a Reimbursement Incentive on Enrollment in a Weight Control Program.” Obesity, 15(11): 2733-2738.

Calfas, Karen J., Barbara J. Long, James F. Sallis, Wilma J. Wooten, Michael Pratt, and Kevin Patrick (1996), "A Controlled Trial of Physician Counseling to Promote the Adoption of Physical Activity”, Preventive Medicine 25(3), May, 225-233.

Camerer, Colin, George Loewenstein, and Drazen Prelec (2005), "Neuroeconomics: How Neuroscience Can Inform Economics”, Journal of Economic Literature, 43(1), March, 964.

Carpenter, Christopher S., Deborah D. Kloska, Patric O’Malley and Lloyd Johnston (2007), "Alcohol Control Policies and Youth Alcohol Consumption: Evidence from 28 Years of Monitoring the Future”, B.E. Journal of Economic Analysis and Poilcy, 7(1 - Topics), Article 25.

Carpenter, Christopher S. and Mark Stehr (2008), “The Effects of Mandatory Seatbelt Laws on Seatbelt Use, Motor Vehicle Fatalities, and Crash-Related Injuries Among Youths”, Journal of Health Economics, 27(3), May, 642-662. 
Carpenter, Christopher and Carlos Dobkin. 2010. “Alcohol Regulation and Crime.” NBER Working Paper No. 15828.

Carpenter, Christopher. 2007. "Heavy Alcohol Use and Crime: Evidence from Underage Drunk Driving Laws.” Journal of Law and Economics, 50(3): 539-557.

Carpenter, Christopher. 2005. "Heavy Alcohol Use and the Commission of Nuisance Crime: Evidence from Underage Drunk Driving Laws,” American Economic Review Papers and Proceedings, 95(2): 267-272.

Carpenter, Christopher, Sabina Postolek, and Casey Warman. 2011. "Public-Place Smokign Laws and Exposure to Environmental Tobacco Smoke (ETS).” Paper presented at the 2011 Annual Meeting on the Economics of Risky Behaviors at IZA in Bonn, Germany.

Carrell, Scott E., Mark Hoekstra and James E. West. 2010. "Is Poor Fitness Contagious? Evidence from Randomly Assigned Friends”, National Bureau of Economic Research Working Paper No. 16518, November.

Casagrande, Sarah Stark, Youfa Wang, Cheryl Anderson, and Tiffany L. Gary (2007) "Have Americans Increased Their Fruit and Vegetable Intake? The Trends Between 1988 and 2002” American Journal of Preventive Medicine 32(4), April, 257-263.

Case, A.C., Katz, L.F., 1991. The company you keep: the effects of family and neighborhood on disadvantaged youth. NBER Working Paper 3705.

Cawley, John. 2000. “Body Weight and Women’s Labor Market Outcomes.” NBER Working Paper \#7841.

Cawley, John. 2004. “The Impact of Obesity on Wages.” Journal of Human Resources, 39(2): 451-474.

Cawley, John. 2008. “Reefer Madness, Frank the Tank or Pretty Woman: To What Extent Do Addictive Behaviors Respond to Incentives?” Chapter 7 in: Frank Sloan and Hirschel Kasper (editors), Incentives and Choice in Health and Health Care, (MIT Press: Cambridge, MA).

Cawley, John. 2010. “The Economics of Childhood Obesity.” Health Affairs, 29(3): 364-371. Cawley, John, and Donald S. Kenkel. 2008. “Introduction.” In: Cawley and Kenkel (eds.), The Economics of Health Behaviours, Volumes 1-3. (Edward Elgar: Northampton, MA). 2008.

Cawley, John and Barrett Kirwan. 2011. “Agricultural Policy and Childhood Obesity” in John Cawley (ed.) Handbook of the Social Science of Obesity. New York: Oxford University Press.

Cawley, John, Euna Han, and Edward C. Norton. Forthcoming.“The Validity of Genes Related to Neurotransmitters as Instrumental Variables.” Health Economics.

Cawley, John and Feng Liu. 2008. "Correlates of State Legislative Action to Prevent Childhood Obesity”, Obesity, 16(1):162-167.

Cawley, John, Sara Markowitz, and John Tauras. 2004. “Lighting Up and Slimming Down: The Effects of Body Weight and Cigarette Prices on Adolescent Smoking Initiation.” Journal of Health Economics, 23(2): 293-311.

Cawley, John and Chad Meyerhoefer. 2010. “The Medical Care Costs of Obesity: An Instrumental Variables Approach.” NBER Working Paper \#16467.

Cawley, John, John Moran, and Kosali Simon. "The Impact of Income on the Weight of Elderly Americans.” Health Economics, 2010, 19(8): 979-993. 
Cawley, John, and Joshua A. Price. 2011. “Outcomes in a Program that Offers Financial Rewards for Weight Loss.” In: Michael Grossman and Naci Mocan (ed.), Economic Aspects of Obesity (Chicago IL: NBER and University of Chicago Press).

Chaloupka, Frank, (1991), "Rational Addictive Behavior and Cigarette Smoking”, Journal of Political Economy, 99(4), August, 722-742.

Chaloupka, Frank J., Henry Saffer, and Michael Grossman. 1993. “Alcohol Control Policies and Motor Vehicle Fatalities” Journal of Legal Studies, 22(1), January, 161-186.

Chaloupka, Frank. 1996. "A Review of Economic Models of Habitual and Addictive Behavior and Their Empirical Applications to Cigarette Smoking.” in Advances in Behavioral Economics. Volume 3, Substance Use and Abuseed. Leonard Green and John H. Kagel. Norwood, N. J.: Ablex Publishing.

Chaloupka, Frank J., Michael Grossman, and John A. Tauras. 1999. “The Demand for Cocaine and Marijuana by Youth.” In Frank J. Chaloupka, Michael Grossman, Warren K. Bickel, and Henry Saffer (eds.), The Economic Analysis of Substance Use and Abuse. (Cambridge, MA: National Bureau of Economic Research), pp. 133-156.

Chaloupka, Frank J. and Kenneth E. Warner. 2000. “The Economics of Smoking.” In A.J. Culyer and J.P Newhouse (eds.) Handbook of Health Economics, Volume 1. (New York: Elsevier), pp. 1539-1628.

Chang, Virginia and Diane S. Lauderdale (2005), “Income Disparities in Body Mass Index in the United States, 1971-2002”, Archives of Internal Medicine, 165(18), October 10, 21222128.

Chapman, G. B., Brewer, N. T., Coups, E. J., Brownlee, S., Leventhal, H. \& Leventhal, E. A. (2001) Value for the future and preventive health behavior. Journal of Experimental Psychology - Applied 7, 235-250.

Charles, Kerwin and Philip DeCicca (2008), "Labor Market Fluctuations and Health: Is There a Connection and for Whom?”, Journal of Health Economics, 27(6), December, 15321550.

Chatterji P. 2006a. Does alcohol use during high-school affect educational attainment? Evidence from the National Education Longitudinal Study. Economics of Education Review 25: 482-497.

Chatterji P. 2006b. Illicit drug use and educational attainment. Health Economics 15: 489-511.

Chen, Keith and Fabian Lange (2008), "Education, Information and Improved Health: Evidence from Cancer Screening”, IZA Discussion Paper No. 3548, June.

Chesson, Harrell, Paul Harrison, William J. Kassler. 2000. "Sex Under the Influence: The Effect of Alcohol Policy on Sexually Transmitted Disease Rates in the United States.” Journal of Law and Economics, April 2000, v. 43, iss. 1, pp. 215-38.

Chetty, Raj; Adam Looney and Kory Kroft. 2009. "Salience and Taxation: Theory and Evidence.” American Economic Review, v. 99, iss. 4, pp. 1145-77.

Chou S-Y, Rashad I, Grossman M. 2008. Fast-food restaurant advertising on television and its influence on childhood obesity. J Law Econ. 51:599-618.

Christakis, N., Fowler, J., 2007. The spread of obesity in a large social network over 32 years. The New England Journal of Medicine 357, 370-379.

Clark, Damon and Heather Royer. 2010. “The Effect of Education on Adult Health and Mortality: Evidence from Britain.” NBER Working Paper \#16013.

Cohen-Cole, Ethan and Jason M. Fletcher. 2008. Is Obesity Contagious? Social Networks vs. Environmental Factors in the Obesity Epidemic. Journal of Health Economics 27 (5): 
1382-1387.

Cohen-Cole, Ethan and Jason M. Fletcher. 2008b. Are All Health Outcomes 'Contagious'? Detecting Implausible Social Network Effects in Acne, Height, and Headaches. British Medical Journal, 3337:a2533.

Conley D. 2009. "The Promise and Challenges of Incorporating Genetic Data into Longitudinal Social Science Surveys and Research,” Biodemography and Social Biology, 55(2): 238251.

Contoyannis, Paul and Andrew M. Jones (2004), "Socio-economic Status, Health and Lifestyle”, Journal of Health Economics, 23(5), September, 965-995.

Cook, Philip J. and George Tauchen. (1982), "The Effect of Liquor Taxes on Heavy Drinking”, Bell Journal of Economics, 13(2), Autumn, 379-390.

Cook, Philip J. 2007. Paying the Tab: The Economics of Alcohol Policy. Princeton University Press: Princeton, NJ.

Cook Philip J, and Michael J. Moore. 1993. “Drinking and schooling”, Journal of Health Economics 12(4), 411-429.

Cook, Philip J. and Michael J. Moore. (2001). "Environment and Persistence in Youthful Drinking Patterns" in Jonathan Gruber (ed.) Risky Behavior Among Youths: An Economic Analysis. Chicago: University of Chicago Press, 375-437.

Cook, Philip J. 1981. "The Effect of Liquor Taxes on Drinking, Cirrhosis and Auto Fatalities" in Alcohol and Public Policy: Beyond the Shadow of Prohibition, Mark H. Moore and Dean R Gerstein (eds.). Washington DC: National Academy Press, 375-437.

Cook, Philip J., and Michael J. Moore. 2000. “Alcohol.” In A.J. Culyer and J.P Newhouse (eds.) Handbook of Health Economics, Volume 1. (New York: Elsevier), pp. 1629-1674.

Cook Philip J, and Michael J. Moore. 2002. "The Economics of Alcohol Abuse and AlcoholControl Policies.” Health Affairs, 21(2): 120-133.

Cook, Philip J., and Michael J. Moore. 1994. "This Tax's for You: The Case for Higher Beer Taxes.” National Tax Journal, September 1994, v. 47, iss. 3, pp. 559-73.

Cook, Philip J.; Ostermann, Jan; Sloan, Frank A. 2005. "The Net Effect of an Alcohol Tax Increase on Death Rates in Middle Age.” American Economic Review, May 2005, v. 95, iss. 2, pp. 278-81.

Courtemanche, Charles (2009), "Longer Hours and Larger Waistlines? The Relationship Between Work Hours and Obesity”, Forum of Health Economics and Policy, 12(2Obesity), Article 2.

Culyer, A.J. and J.P. Newhouse. (2000) “The State and Scope of Health Economics.” In: Culyer and Newhouse (eds.) Handbook of Health Economics, Volume 1A. (New York: Elsevier), pp. 1-8.

Cummings, K.M., A. Hyland, T.F. Pechacek, M. Orlandi, and W.R. Lynn. 1997. “Comparison of recent trends in adolescent and adult cigarette smoking behaviour and brand preferences.” Tobacco Control, 6(suppl 2): S31-S37.

Currie, Janet, and Enrico Moretti. 2003. "Mother education and the intergenerational transmission of human capital: evidence from college openings and longitudinal data." Quarterly Journal of Economics, 118(4), 1495-1532.

Cutler, David M. and Adriana Lleras-Muney (2010), "Understanding Differences in Health Behaviors By Education”, Journal of Health Economics, 29(1), January, 1-28.

Cutler, David M., Edward L. Glaeser and Jesse M. Shapiro (2003), "Why Have Americans Become more Obese?”, Journal of Economic Perspectives, 17(3), Summer, 93-118. 
Cutler, David M. and Edward Glaeser (2005), "What Explains Differences in Smoking, Drinking, and Other Health-Related Behaviors”, American Economic Review, 95(2), 238-242.

Cutler, David M., Edward L. Glaeser and Alison B. Rosen (2009), "Is the US Population Behaving Healthier” in Jeffrey R. Brown, Jeffrey B. Liebman and David A. Wise (eds.), Social Security Policy in a Changing Environment (University of Chicago Press, Chicago), 423-441.

Danaei G, Ding EL, Mozaffarian D, Taylor B, Rehm J, et al. (2009) The Preventable Causes of Death in the United States: Comparative Risk Assessment of Dietary, Lifestyle, and Metabolic Risk Factors. PLoS Med 6(4): e1000058. doi:10.1371/journal.pmed.1000058.

Dave, Dhaval and Robert Kaestner. 2002. “Alcohol Taxes and Labor Market Outcomes.” Journal of Health Economics, May 2002, v. 21, iss. 3, pp. 357-71.

Dave, Dhaval and Robert Kaestner. 2009. "Health Insurance and Ex Ante Moral Hazard:

Evidence from Medicare”, International Journal of Health Care Finance and Economics, 9(4), December, 367-390.

DeCicca, Philip, Donald Kenkel, and Alan Mathios (2002). "Putting Out the Fires: Will Higher Taxes Reduce the Onset of Youth Smoking?” Journal of Political Economy 110 (1): 144169.

DeCicca, Philip, Donald Kenkel, and Alan Mathios (2008). "Cigarette Taxes and the Transition from Youth to Adult Smoking: Smoking Initiation, Cessation, and Participation." Journal of Health Economics 27 (4): 904-917.

DeCicca, Phillip, Donald Kenkel, Alan Mathios, Yoon-Jeong Shin, and Jae-Young Lim (2008). "Youth Smoking, Cigarette Prices, and Anti-Smoking Sentiment." Health Economics 17 (6): 733-749.

Decker, Sandra L. 2005. “Medicare and the Health of Women”, Journal of Human Resources, 40(4), 948-968.

Dee, Thomas S., and William N. Evans. 2003. Teen drinking and educational attainment: evidence from two-sample instrumental variables estimates. Journal of Labor Economics 21: 178-209.

Dee, Thomas S.. 1999. “State Alcohol Policies, Teen Drinking and Traffic Fatalities,” Journal of Public Economics, 72(2): 289-315.

Dee, Thomas S. (2001), “Alcohol Abuse and Economic Conditions: Evidence From Repeated Cross-Sections of Individual-Level Data”, Health Economics 10(3), March, 257-70.

Dee, Thomas S. (2009), "Motorcycle Helmets and Traffic Safety", Journal of Health Economics, 28(2), March, 398-412.

Dehejia, Rajeev and Adriana Lleras-Muney (2004), “Booms, Busts, and Babies’ Health”, Quarterly Journal of Economics 119(3), August, 1091-1130.

Della Vigna, Stefano and Ulrike Malmendier (2006), "Paying Not to Go to the Gym”, American Economic Review, 96(3), June, 674-719.

DeSimone J. 2002. Illegal drug use and employment. Journal of Labor Economics 20: 952-977.

De Walque, Damien (2007a), "How Does the Impact of an HIV/AIDS Information Campaign Vary With Educational Attainment? Evidence from Uganda”, Journal of Development Economics, 84(2), November, 686-714.

De Walque, Damien (2007b), "Does Education Affect Smoking Behaviors? Evidence Using the Vietnam Draft as an Instrument for College Education”, Journal of Health Economics, 27(5), September, 877-895. 
Dills, Angela K., Mireille Jacobson, and Jeffrey A. Miron. 2005. “The Effect of Alcohol Prohibition on Alcohol Consumption: Evidence from Drunkenness Arrests.” Economics Letters, 86(2005): 279-284.

Ding, Weili, Steven F. Lehrer, J. Niels Rosenquist, and Janet Audrain-McGovern. 2009. "The impact of poor health on academic performance: New evidence using genetic markers.” Journal of Health Economics 28 (2009) 578-597.

Ding, Weili, Steven F. Lehrer, J. Niels Rosenquist, and Janet Audrain-McGovern (2009), “The Impact of Poor Health on Academic Performance: New Evidence Using Genetic Markers”, Journal of Health Economics 28(3), May, 578-597.

Dixon, John B. (2010) The effect of obesity on health outcomes. Molecular and Cellular Endocrinology 316: 104-108.

Dobkin, Carlos and Steven Puller (2007), “The Effects of Government Transfers on Monthly Cycles in Drug Abuse, Hospitalization and Mortality”, Journal of Public Economics, 91(11-12), December, 2137-2157.

Dobkin, Carlos and Nancy Nicosia. 2009. “The War on Drugs: Methamphetamine, Public Health, and Crime.” American Economic Review, 99(1): 324-349.

Dockner, Engelbert J. and Gustav Feichtinger, (1993), “Cyclical Consumption Patterns and Rational Addiction” American Economic Review 83(1), March, 256-263.

Dragone, Davide (2009), “A Rational Addiction Model of Binges, Diets and Obesity”, Journal of Health Economics, 28(4), July, 799-804.

Dranove, D.,Wehner, P., 1994. Physician-induced demand for childbirths. Journal of Health Economics 13, 61-73.

Dube, S.R., K. Asman, A. Malarcher, and R. Carbollo (2009), “Cigarette Smoking Among Adults and Trends in Smoking Cessation - United States, 2008” Morbidity and Mortality Weekly Report, 58(44), November 13, 1227-1232.

Duncan, Greg J., Johanne Boisjoly, Michael Kremer, Dan M. Levy, and Jacque Eccles. 2005. "Peer Effects in Drug Use and Sex Among College Students." Journal of Abnormal Child Psychology, 33(3): 375-385.

Dupas, Pascaline. 2011. "Do Teenagers Respond to HIV Risk Information? Evidence from a Field Experiment in Kenya.” American Economic Journal: Applied Economics, 3(1): 134.

Edwards, Ryan (2008), “American Time Use Over the Business Cycle”, mimeo, Queens College, August.

Ettner, Susan L. (1997), "Measuring the Human Cost of A Weak Economy: Does Unemployment Lead to Alcohol Abuse?”, Social Science and Medicine, 44(2), January, 251-60.

Evans, William and John D. Graham (1988), “Traffic Safety and the Business Cycle”, Alcohol, Drugs, and Driving 4(1), January-March, 31-38.

Evans, William N. and Matthew C. Farrelly. 1998. "The Compensating Behavior of Smokers: Taxes, Tar, and Nicotine.” Rand Journal of Economics, 29(3): 578-595.

Evans, William N. and Timothy J. Moore. Forthcoming. "Liquidity, Activity and Mortality,” Review of Economics and Statistics.

Fang, Hanming and Alessandro Gavazza. 2007. "Dynamic Inefficiencies in an EmploymentBased Health Insurance System: Theory and Evidence”, National Bureau of Economic Research Working Paper No. 13371, September.Farrell, P., Fuchs, V.R., 1982. Schooling and health: the cigarette connection. Journal of Health Economics, 1: 217-230. 
Farrelly, M.C., C.T. Nimsch, A. Hyland, and M. Cummings. 2004. "The ejects of higher cigaretteprices ontar and nicotine consumption in a cohort of adult smokers.” Health Economics, 13: 49-58.

Farrelly, Matthew C., Jeremy W. Bray, Gary A. Zarkin, and Brett W. Wendling. 2001. "The joint demand for cigarettes and marijuana: evidence from the National Household Surveys on Drug Abuse,” Journal of Health Economics, 20(1): 51-68.

Feng W, Zhou W, Butler JS, Booth BM, French MT. 2001. The impact of problem drinking on employment. Health Economics 10: 509-521.

Ferguson, Brian. 2000. "Interpreting the Rational Addiction Model,” Health Economics, 9(7): 587-98.

Fernald, Lisa C.H., Paul J. Gertler, and Lynnette M. Neufeld. 2008. "Role of cash in conditional cash transfer programmes for child health, growth, and development: an analysis of Mexico's Oportunidades.” Lancet, 371: 828-837.

Fernald, Lisa C.H., Paul J. Gertler, and Xiaohui Hou. 2008. "Cash Component of Conditional Cash Transfer Program Is Associated with Higher Body Mass Index and Blood Pressure in Adults.” Journal of Nutrition, 138: 2250-2257.

Finkelstein, Eric A., Trogdon JG, Cohen JW, Dietz W. Annual medical spending attributable to obesity: payer- and service-specific estimates, Health Affair Web Exclusive. July 27 2009.

Finkelstein, Eric A., Linnan, L.A., Tate, D.F., Birken, B.E. (2007) “A Pilot Study Testing the Effect of Different Levels of Financial Incentives on Weight Loss Among Overweight Employees.” Journal of Occupational and Environmental Medicine. 49(9): 981-989.

Flegal, Katherine M., Barry I. Graubard, David F. Williamson and Mitchell H. Gail (2005), "Excess Deaths Associated with Underweight, Overweight, and Obesity", JAMA, 293(15), April 20, 1861-1867.

Flegal, Katherine M., Barry I. Graubard, David F. Williamson and Mitchell H. Gail (2007), "Cause-Specific Excess Deaths Associated With Underweight, Overweight, and Obesity,” JAMA, 298(17): 2028-2037.

Fletcher, Jason M. and Steven F. Lehrer. 2009. "The Effects of Adolescent Health on Educational Outcomes: Causal Evidence Using Genetic Lotteries between Siblings.” Forum for Health Economics \& Policy, 12(2): article 8.

Forster, Martin, and Andrew M. Jones. 2001. "The Role of Tobacco Taxes in Starting and Quitting Smoking: Duration Analysis of British Data.” Journal of the Royal Statistical Society, 164(Part 3): 517-547.

Fowler, JH and NA Christakis. 2008. Estimating peer effects on health in social networks: A response to Cohen-Cole and Fletcher; and Trogdon, Nonnemaker, and Pais. Journal of Health Economics, 27: 1400-1405.

Frank, Mark W. 2008. "Media substitution in advertising: A spirited case study.” International Journal of Industrial Organization, 26: 308-326.

Frederick, Shane, George Loewenstein and Ted O’Donoghue, (2002), “Time Discounting and Time Preference: A Critical Review”, Journal of Economic Literature, 40(2), June, 351401.

Fredriksson, Per G.; Gohmann, Stephan; Mamun, Khawaja. 2009. "Taxing under the Influence? Corruption and U.S. State Beer Taxes.” Public Finance Review, 37(3): 339-65.

Freeman, Donald G (1999), “A Note on 'Economic Conditions and Alcohol Problems”, Journal of Health Economics 18(5), October, 661-670. 
French, Michael T. and Johanna C. Maclean. 2006. "Underage alcohol use, delinquency, and criminal activity.” Health Econ. 15: 1261-1281.

French, Michael T, McGeary KA, Chitwood DD, McCoy CB. 2000. Chronic illicit drug use, health services utilization, and the cost of medical care. Social Science and Medicine 50: 1703-1713.

French, Michael T, Roebuck MC, Alexandre PK. 2001. Illicit drug use, employment, and labor force participation. Southern Economic Journal 68: 349-368.

French, Michael T. and Ioana Popovici. 2011. “That instrument is lousy! In search of agreement when using instrumental variables estimation in substance use research.” Health Economics, 20: 127-146.

Fryer Jr., Roland G., Paul S. Heaton, Steven D. Levitt, and Kevin M. Murphy. 2005. "Measuring the Impact of Crack Cocaine.” NBER Working Paper \#11318.

Fuchs, V.R., 1982. Time preference and health: an exploratory study. In: Fuchs, V.R. (Ed.), Economic Aspects of Health. University of Chicago Press for the NBER, pp. 93-120.

Fudenberg, Drew and David K. Levin, (2006), “A Dual-Self Model of Impulse Control”, American Economic Review, 96(5), December, 1449-1476.

Gallet, Craig A.; Hoover, Gary A.; Lee, Junsoo. 2006. "Putting Out Fires: An Examination of the Determinants of State Clean Indoor-Air Laws.” Southern Economic Journal, v. 73, iss. 1, pp. 112-24.

Gallet, C., List, J. A. 2003. Cigarette Demand: A Meta -Analysis of Elasticities. Health Economics, 12: 821-835.

Gallet, Craig A. 2007. “The Demand for Alcohol: A Meta-Analysis of Elasticities.” Australian Journal of Agricultural and Resource Economics, 51: 121-135.

Gasmi, F., J.J. Laffont, and Q. Vuong. 1992. "Econometric Analysis of Collusive Behavior in a Soft Drink Market.” Journal of Economics \& Management Strategy, 1(2): 277-311.

Gaziano, J. Michael , Julie E. Buring, Jan L. Breslow, Samuel Z. Goldhaber, Bernard Rosner, Martin VanDenburgh, Walter Willett, and Charles H. Hennekens, (1993), "Moderate Alcohol Intake, Increased Levels of High-Density Lipoprotein and Its Subfractions, and Decreased Risk of Myocardial Infarction” New England Journal of Medicine, 329(25), September 16, 1829-1834.

Gertler, Paul, Manisha Shah, Stefano M. Bertozzi (2005), "Risky Business: The Market for Unprotected Commercial Sex”, Journal of Political Economy, 113(3), June, 518-550.

Gilleskie, Donna. “A Dynamic Stochastic Model of Medical Care Use and Work Absence.” Econometrica 66(1), Jan 1998, p. 1-45.

Gilleskie, Donna and Koleman Strumpf. 2005. “The Behavioral Dynamics of Youth Smoking” Journal of Human Resources, 40(4): 822-866.

Giné, Xavier, Dean Karlan, and Jonathan Zinman. 2010. "Put Your Money Where Your Butt Is: A Commitment Contract for Smoking Cessation.” American Economic Journal: Applied Economics, 2: 213-235.

Giuffrida, Antonio and David J. Torgerson. 1997. "Should we Pay the Patient? Review of Financial Incentives to Enhance Patient Compliance.” British Medical Journal, 315: 703707.

Glimcher, Paul William, Joseph Kable and Kenway Louie (2007), “Neuroeconomic Studies of Impulsivity: Now or Just as Soon as Possible”, American Economic Review, 97(2), May, 142-147. 
Goldman, Dana P. and James P. Smith (2002), "Can Patient Self-Management Help Explain the SES Health Gradient”, Proceedings of the National Academy of Sciences, 99(16), August 6, 10929-10934.

Goldman, David, Gabor Oroszi and Francesca Ducci (2005), “The Genetics of Addictions: Uncovering the Genes”, Nature Reviews: Genetics, 67(6), July, 521-532.Goldman, Dana, Darius Lakdawalla, and Yuhui Zheng. 2009. "Food Prices and the Dynamics of Body Weight” National Bureau of Economic Research Working Paper No. 15096, June.

Gregory Christian A. and Christopher J. Ruhm. 2011. "Where Does the Wage Penalty Bite”, in Michael Grossman and Naci Mojan (eds.) Economic Aspects of Obesity, University of Chicago Press

Greve, Jane. 2008. “Obesity and labor market outcomes in Denmark.” Economics and Human Biology, 6: 350-362.

Grimard, Franque and Daniel Parent (2007), "Education and Smoking: Were Vietnam War Draft Avoiders Also More Likely to Avoid Smoking?”, Journal of Health Economics, 27(5), September, 896-926.

Grogger, Jeff, and Michael Willis. 2000. "The Emergence of Crack Cocaine and the Rise in Urban Crime Rates,” Review of Economics and Statistics, 82(4): 519-529.

Grossman, Michael. 1972. "On the Concept of Health Capital and the Demand for Health.” Journal of Political Economy, 80(2): 223-249.

Grossman, Michael. “The Demand for Health After a Decade,” 1982, Journal of Health Economics 1(1): 1-3.

Grossman, Michael. 1993. “The Economic Analysis of Addictive Behavior.” in Economics and the Prevention of Alcohol-Related Problems, ed. Michael E. Hilton and Gregory Bloss. Rockville, MD: U. S. Department of Health and Human Services.

Grossman, Michael. 2000. “The Human Capital Model.” In A.J. Culyer and J. P. Newhouse (eds.), Handbook of Health Economics, volume 1A (New York: Elsevier).

Grossman, Michael. 2005. "Individual Behaviours and Substance Use: The Role of Price,” Advances in Health Economics and Health Services Research, 16: 15 - 39.

Grossman, Michael, and Frank J. Chaloupka. 1998. “The Demand for Cocaine by Young Adults: A Rational Addiction Approach,” Journal of Health Economics, 17(4): 427-74.

Grossman, M., and R. Kaestner. 1997. "Effects of Education on Health. In The Social Benefits of Education,” In: J. R. Behrman and N. Stacey (eds.), Ann Arbor, Mich.: University of Michigan Press.

Gruber, Jonathan and Botond Köszegi (2001), “Is Addiction ‘Rational’? Theory and Evidence”, Quarterly Journal of Economics, November, 116(4), 1261-1303.

Gruber, Jonathan, and B. Koszegi. 2004. "Tax Incidence When Individuals Are TimeInconsistent: The Case of Cigarette Excise Taxes.” Journal of Public Economics, 88: 1959-1987.

Gruber, Jonathan and Sendhil Mullainathan. 2005. “Do Cigarette Taxes Make Smokers Happier?” Advances in Economic Analysis and Policy, 5(1), Article 4.

Gruber, Jonathan and Michael Frakes (2006), “Does Falling Smoking Lead to Rising Obesity?”, Journal of Health Economics, 25(2), March, 183-197.

Gul, Faruk and Wolfgang Pesendorfer (2001), “Temptation and Self-Control”, Econometrica, 69(6), November, 1403-1435.

Hall, Robert E. (1978), "Stochastic Implications of the Lifecycle-Permanent Income Hypothesis: Theory and Evidence”, Journal of Political Economy, 86(6), December, 971-987. 
Hamermesh, Daniel S. and Jeff E. Biddle. 1994. "Beauty and the Labor Market”, American Economic Review 84(5), 1174-1194.

Hare, Todd A., Colin F. Camerer and Antonio Rangel (2009), "Self-Control in Decision-Making Involves Modulation of the vmPFC Valuation System”, Science, 324, May 1, 646-648.

Harwood, Hendrick. 2000. Updating estimates of the economic costs of alcohol abuse in the United States: estimates, update methods, and data. National Institute on Alcohol Abuse and Alcoholism.

Hastings, Justine and Ebonya Washington (2010), “The First of the Month Effect: Consumer Behavior and Store Responses”, American Economic Journal: Economic Policy, 2(2), May, 142-162.

Heckman, James J., Flyer, Fredrick and Loughlin, Colleen P. 2008. An Assessment of Causal Inference in Smoking Initiation Research and a Framework for Future Research. Economic Inquiry, 46(1): 37-44.

Higgins, Stephen T., Sheila M. Alessi, and Robert L. Dantona. 2002. "Voucher-Based Incentives: A Substance Abuse Treatment Innovation.” Addictive Behaviors, 27: 887910.

Houthakker, H. S., and Taylor, L. D. (1970), Consumer Demand in the United States: Analyses and Projections, Cambridge, MA: Harvard University Press.

Ippolito, Pauline M. and Alan D. Mathios. 1995. "Information and Advertising: The Case of Fat Consumption in the United States.” American Economic Review, 85(2): 91-95.

Johansson, E, Alho H, Kiiskinen U, Poikolainen K. 2007. The association of alcohol dependency with employment probability: evidence from the population survey 'Health 2000 in Finland.' Health Economics 16: 739-754.

Johansson, Edvard, Petri Böckerman, Urpo Kiiskinen, and Markku Heliövaara (2009), “Obesity and Labour Market Success in Finland: The Difference Between Having a High BMI and Being Fat”, Economics and Human Biology, 7(1), March, 36-45.

Johnston, L.D., P.M. O’Malley, J.G. Bachman and J.E. Schulenberg (2009), “Teen Marijuana Use Tilts Up, While Some Drugs Decline in Use” (University of Michigan News Service, Ann Arbor, MI), retrieved from www.monitoringthefuture.org, 4/19/2010.

Just, David R. (2006), “Behavioral Economics, Food Assistance, and Obesity”, Agricultural and Resource Economics Review, 35(2), October, 209-220.

Kaestner, Robert. 1991. “The Effect of Illicit Drug Use on the Wages of Young Adults.” Journal of Labor Economics, 9(4): 381-412.

Kaestner, Robert. 1994. "New Estimates of the Effect of Marijuana and Cocaine Use on Wages,” Industrial and Labor Relations Review, 47(3): 454-470.

Kahneman, Daniel (1994), "New Challenges to the Rationality Assumption”, Journal of Institutional and Theoretical Economics, 150(1), 18-36.

Kahneman, Daniel, and Richard H. Thaler. 2006. “Anomalies: Utility Maximization and Experienced Utility.” Journal of Economic Perspectives, 20(1): pp. 221-234.

Kahneman, Daniel, Jack L. Knetsch, Richard Thaler. 1986. "Fairness as a Constraint on Profit Seeking: Entitlements in the Market.” American Economic Review, 76(4): 728-741.

Kahneman, D. and Tversky, A. (1979). "Prospect Theory: An Analysis of Decision Under Risk. Econometrica. 47(2): 263-292.

Kan, Kamhon (2007), “Cigarette Smoking and Self-Control”, Journal of Health Economics 26(1), January, 61-81. 
Kanjilal, Sanjat, Edward W. Gregg, Yiling J. Cheng, Ping Zhang, David E. Nelson, Geoge Mensah and Gloria L.A. Beckles (2006), "Socioeconomic Status and Trends in Disparities in 4 Major Risk Factors for Cardiovascular Disease Among US Adults”, Archives of Internal Medicine, 166(21), November 27, 2348-2355.

Kant, Ashima and Barry Graubard (2007), "Secular Trends in the Association of Socio-economic Position with Self-Reported Dietary Attributes and Biomarkers in the US Population: National Health and Nutrition Examination Survey (NHANES) 1971-1975 to NHANES 1999-2002”, Public Health Nutrition, 10(2), February, 158-167.

Kenkel, Donald S. (1991) “Health Behavior, Health Knowledge and Schooling” Journal of Political Economy, 99(2), April, 287-305.

Kenkel, Donald S. 2000. "Prevention.” In A.J. Culyer and J.P Newhouse (eds.) Handbook of Health Economics, Volume 1. (New York: Elsevier), pp. 1675-1720.

Kenkel, Donald S. 2005. “Are Alcohol Tax Hikes Fully Passed through to Prices? Evidence from Alaska.” American Economic Review, 95(2): 273-277.

Kenkel, Donald S. 2006. "Health behaviours among young people.” Chapter 6 in: Andrew M. Jones (Editor), The Elgar Companion to Health Economics. Edward Elgar: Northampton, MA.

Kenkel, Donald, Lillard, Dean, \& Mathios, Alan. 2006. “The Roles of High School Completion and GED Receipt in Smoking and Obesity.” Journal of Labor Economics, 24(3), 635660.

Kenkel, Donald S. (2007), “The Evolution of the Schooling-Smoking Gradient”, mimeo, Cornell University, March.

Kenkel, Donald S. and Jody Sindelar. Forthcoming. "Economics of Health Behaviors and Addictions: Contemporary Issues and Policy.” In: Sherry Glied (editor), Oxford Handbook of Health Economics, Oxford University Press: New York, NY.

Khwaja, Ahmed, Dan Silverman, Frank Sloan, and Yang Wang (2009), “Are Mature Smokers Misinformed”, Journal of Health Economics, 28(2), March, 385-397.

Khwaja, Ahmed, Frank Sloan, and Sukyung Chung (2007), “The Relationship Between Individual Expectations and Behaviors: Mortality Expectations and Smoking Decisions”, Journal of Risk and Uncertainty, 35(2), October, 179-201.

Klick, Jonathan and Thomas Stratmann. 2007. "Diabetes Treatments and Moral Hazard”, Journal of Law and Economics, 50(3), August, 519-538.

Kline, Brendan and Justin L. Tobias. 2008. "The wages of BMI: Bayesian analysis of a skewed treatment-response model with nonparametric endogeneity.” Journal of Applied Econometrics, 23: 767-793.

Kling, Jeffrey R, Jeffrey B Liebman, and Lawrence F Katz. 2007. "Experimental Analysis of Neighborhood Effects.” Econometrica, 75(1): 83-119.

Koch, S. F., \& Ribar, D. C. 2001. “A siblings analysis of the effects of alcohol consumption onset on educational attainment.” Contemporary Economic Policy, 19(2): 162-174.

Komlos, John, Patricia K. Smith, and Barry Bogin. (2004) "Obesity and the Rate of Time Preference: Is there a Connection?” J. biosoc. Sci., 36, 209-219.

Laibson, David (2001) “A Cue-Theory of Consumption”, Quarterly Journal of Economics, 116(1), February, 81-119.

Laibson, D. (1997). “Golden Eggs and Hyperbolic Discounting.” Quarterly Journal of Economics. 112(5): 443-477. 
Lakdawalla D, Philipson T, Bhattacharya J. 2005. Welfare-enhancing technological change and the growth of obesity. American Economic Review 95(2): 253-257.

Lange, Fabian (2010), "Does Education Help with Complex Health Decisions: Evidence from Cancer Screening”, mimeo, Yale University.

Lantz, Paula M., John W. Lynch, James S. House, James M. Lepkowski, Richard P. Mero, Marc A. Musick and David R. Williams (2001), "Socioeconomic Disparities in Health Change in a Longitudinal Study of US Adults: the Role of Health-risk Behaviors”, Social Science and Medicine, 53(1), July, 29-40.

Lawrance, E. (1991) Poverty and the rate of time preference: evidence from panel data. Journal of Political Economy 99(1), 54-77.

Leibenstein, H. 1950. "Bandwagon, Snob, and Veblen Effects in the Theory of Consumers' Demand.” Quarterly Journal of Economics, 64(2): 183-207.

Lerner, Jennifer S. and Dacher Keltner. 2001. "Fear, Anger, and Risk” Journal of Personality and Social Psychology, 81(1), July, 146-159.

Levine, Phillip B. (2001), "The Sexual Activity and Birth-Control Use of American Teenagers”, in Jonathan Gruber (ed.), Risky Behavior Among Youths. Chicago: The University of Chicago Press, 167-217.

Levy, Douglas E. and Ellen Meara. 2006. "The Effect of the 1998 Master Settlement Agreement on Prenatal Smoking.” Journal of Health Economics, 25: 276-294.

Lindahl, Mikael. 2005. "Estimating the Effect of Income on Health and Mortality Using Lottery Prizes as an Exogenous Source of Variation in Income.” Journal of Human Resources, 40(1): 144-168.

Liu, Jin-Long, Jin-Tan Liu, James K. Hammitt, and Shin-Yi Chou. 1999. "The price elasticity of opium in Taiwan, 1914-1942.” Journal of Health Economics, 18: 795-810.

Lleras-Muney, Adriana and Frank Lichtenberg, (2005), "The Effect Of Education On Medical Technology Adoption: Are The More Educated More Likely To Use New Drugs?", Annales d'Economie et Statistique, 79/80, July-December.

Loewenstein G, Brennan T, Volpp KG. 2007. "Asymmetric paternalism to improve health behaviors.” JAMA, 298(20): 2415-7.

Loewenstein, George (2000), "Emotions in Economic Theory and Economic Behavior", American Economic Review, 90(2), 426-432.

Loewenstein, George F. and O'Donoghue, Ted (2004), “Animal Spirits: Affective and Deliberative Processes in Economic Behavior”, Working Paper 04-14, Center for Analytic Economics, Cornell University.

Loomis, B.R., M.C. Farrelly, J.M. Nonnemaker and N.H. Mann (2006), "Point of Purchase Cigarette Promotions Before and After the Mast Settlement Agreement: Exploring Retail Scanner Data” Tobacco Control 15(2), April, 140-142.

Lundborg, P., 2006. Having the wrong friends? Peer effects in adolescent substance use. Journal of Health Economics 25(2): 214-233.

Lundborg, P., K. Bolin, S. Hojgard, and B. Lindgren. 2007. Obesity and occupational attainment among the 50+ of Europe. In: Bolin, K., Cawley, J. (Eds.), Advances in Health and Health Services Research, Vol. 17: The Economics of Obesity. Elsevier, New York.

Lussier, Jennifer Plebani, Sarah H. Heil, Joan A. Mongeon, et al. 2006. "A Meta-Analysis of Voucher-Based Reinforcement Therapy for Substance Use Disorders.” Addiction, 101: 192-203. 
Lye, Jenny and Joe Hirschberg. 2010. “Alcohol Consumption and Human Capital: A Retrospective Study of the Literature.” Journal of Economic Surveys, 24: 309-338.

Lynch, John W., George A. Kaplan, Richard D. Cohen, Jaakko Tuomilehto and Jukka T. Salonen (1996), "Do Cardiovascular Risk Factors Explain the Relation Between Socioeconomic Status, Risk of All-Cause Mortality, Cardiovascular Mortality and Acute Myocardial Infarction”, American Journal of Epidemiology, 144(10), November 15, 934-942.

MacDonald, Ziggy and Stephen Pudney. 2000. “The Wages of Sin? Illegal Drug Use and the Labour Market.” Labour, 14(4): 657-674.

MacLean, Paul D. (1990), The Triune Brain in Evolution: Role in Paleocerebral Functions. New York: Plenum.

Maitra, Sudeshna (2010), “Can Patient Self-Management Help Explain the SES Health Gradient? Goldman and Smith’s 'Can Patient Self-Management Help Explain the SES Health Gradient?’ (2002) Revisited”, Social Science and Medicine, 70(6), March, 802810.

Manning, Willard G., Emmett B. Keeler, Joseph P. Newhouse, Elizabeth M. Sloss, and Jeffrey Wasserman. 1991. The Costs of Poor Health Habits (Cambridge MA: Harvard University Press).

Manning, Willard G., Linda Blumberg, and Lawrence H. Moulton. 1995. "The Demand for Alcohol: The Differential Response to Price,” Journal of Health Economics, 14(2): 12348.

Manski, C.F., 1993. Identification of endogenous social effects: the reflection problem. Review of Economic Studies 60-3, 531-542.

Manski, Charles F. 2000. “Economic Analysis of Social Interactions.” Journal of Economic Perspectives, 14(3): 115-136.

Markowitz, Sara. 2005. "Alcohol, Drugs and Violent Crime," International Review of Law and Economics, 25(1): 20-44.

Markowitz, Sara and Michael Grossman. 2000. "The Effects of Beer Taxes on Physical Child Abuse.” Journal of Health Economics, v. 19, iss. 2, pp. 271-82.

Markowitz, Sara; Pinka Chatterji and Robert Kaestner. 2003. "Estimating the Impact of Alcohol Policies on Youth Suicides.” Journal of Mental Health Policy and Economics, March 2003, v. 6, iss. 1, pp. 37-46.

Marmot, M.G., Geoffrey Rose, M. Shipley, and P.J.S. Hamilton (1978), “Employment Grade and Coronary Heart Disease in British Civil Servants” Journal of Epidemiology and Community Health, 32(4), December, 244-249.

Marmot, M.G., George Davey Smith, Stephen Stansfeld, Chandra Patel, Fiona North, Jenny Head, et. al. (1991), "Health Inequalities Among British Civil Servants: The Whitehall II Study”, The Lancet, 337(8754), June 8, 1387-1393.

Marmot, Michael and Richard G. Wilkinson. 2006. Social Determinants of Health (2 ${ }^{\text {nd }}$ edition). Oxford: Oxford University Press.

Marshall, Alfred. (1920) Principles of Economics, 8th edition, London.

Massey, Douglas S. (2002), “A Brief History of Human Society: The Origin and Role of Emotion in Social Life”, American Sociological Review, 67(1), February, 1-29.

Mast, Brent D., Bruce L. Benson, and David Rasmussen (1999), "Beer Taxation and AlcoholRelated Fatalities”, Southern Economic Journal, 66(20, October, 214-249. 
Mastrobuoni, Giovanni and Matthew Weinberg (2009), "Heterogeneity in Intra-Monthly Consumption Patterns, Self-Control and Savings at Retirement”, American Economic Journal: Economic Policy, 1(2), August, 163-189.

Mas-Colell, Andreu; Whinston, Michael D.; and Jerry R. Green (1995). Microeconomic Theory. New York: Oxford University Press.

Mathios, Alan. 2000. “The Impact of Mandatory Disclosure Laws on Product Choices: An Analysis of the Salad Dressing Market.” Journal of Law and Economics, 43(2): 651-77.

McCloskey, D.N. 1998. The Rhetoric of Economics, University of Wisconsin Press; 2nd ed.

McClure, Samuel M., David I. Laibson, Goerge Loewenstein and Jonathan D. Cohen (2004), “Separate Neural Systems Value Immediate and Delayed Monetary Rewards”, Science, 306, October 15, 503-507.

McDowell, MA, C-Y Wang, and J. Kennedy-Stephenson (2006), Breastfeeding in the United States: Findings from the National Health and Nutrition Examination Surveys 19992006. (National Center for Health Statistics, Hyattsville, MD).

McGeary KA, French MT. 2000. Illicit drug use and emergency room utilization. Health Services Research 35: 153-169.

McGinnis, J. Michael and William H. Foege (1993), “Actual Causes of Death in the United States”, JAMA 270(18), November 10, 2207-2212.

Metcalfe, Janet and Walter Mischel. 1999. “A Hot/Cool - System Analysis of Delay of Gratification: Dynamics of Willpower”, Psychological Review, 106(1), 3-19.

Miron, Jeffrey A. and Jeffrey Zwiebel. 1991. "Alcohol Consumption During Prohibition.” American Economic Review, 81(2): 242-247.

Miron, Jeffrey A. (2003), "Do Prohibitions Raise Prices? Evidence from the Markets for Cocaine and Heroin”, Review of Economics and Statistics, 85(3), 522-530.

Mokdad, Ali H., James S. Marks, Donna F. Stroup and Julie L. Gerberding (2004), “Actual Causes of Death in the United States, 2000”, JAMA 291(10), March 10, 1238-1245.

Mokdad, Ali H., James S. Marks, Donna F. Stroup, and Julie L. Gerberding. 2005. “Correction: Actual Causes of Death in the United States, 2000.” JAMA 293(3): 293.

Morris, Stephen. 2007. “The impact of obesity on employment.” Labour Economics 14 (2007) 413-433.

Morrisey, Michael A., and John Cawley. (2008) "U.S. Health Economists: Who We Are and What We Do.” Health Economics, 17(4): 535-543.

Mullahy J, Sindelar J. 1996. Employment, unemployment, and problem drinking. Journal of Health Economics 15: 409-434.

Mullahy, John (1999), "It Will Only Hurt for a Second? Microeconomic Determinants of Who Gets Flu Shots” Health Economics, 8(1), February, 9-24.

National Center for Health Statistics (2010), Health United States, 2009: With Special Feature on Medical Technology. (National Center for Health Statistics, Hyattsville, MD).

Nelson, Philip. 1974. “Advertising as Information.” Journal of Political Economy, 81: 729-754.

Nelson, Jon P. 2003. "Cigarette Demand, Structural Change, and Advertising Bans: International Evidence, 1970-1995.” Contributions to Economic Analysis \& Policy, 2(1): Article 10.

Nelson, Jon P. 2006. “Cigarette Advertising Regulation: A Meta-Analysis.” International Review of Law and Economics, 26: 195-226.

Nelson, Jon P. 2010. "Alcohol advertising bans, consumption and control policies in seventeen OECD countries, 1975-2000.” Applied Economics, 42(7): 803-823. 
Norton, Edward C. and Euna Han (2008), “Genetic Information, Obesity, and Labor Market Outcomes”, Health Economics 17(9), September, 1089-1104.

O’Donoghue, Ted and Matthew Rabin (1999), “Doing it Now or Later”, American Economic Review, 89(1), March, 103-124.

Oettinger, Gerald S. (1999), "The Effects of Sex Education on Teen Sexual Activity and Teen Pregnancy”, Journal of Political Economy, 107(3), June, 606-635.

Office of National Drug Control Policy (2004). The Economic Costs of Drug Abuse in the United States, 1992-2002. Washington, DC: Executive Office of the President (Publication No. 207303).

Ogburn, William F. and Dorothy S. Thomas (1922), “The Influence of the Business Cycle on Certain Social Conditions”, Journal of the American Statistical Association, 18(139), September, 324-40.

Ohsfeldt, Robert L.; Morrisey, Michael A. 1997. “Beer Taxes, Workers' Compensation, and Industrial Injury.” Review of Economics and Statistics, v. 79, iss. 1, pp. 155-60.

Olshansky S. Jay, Douglas J. Passaro, Ronald C. Hershow, Jennifer Layden, Bruce A. Carnes, Jacob Brody J, et al. (2005), “A Potential Decline in Life Expectancy in the United States in the 21st Century”, New England Journal of Medicine, 352(11), March 17, 1138-1145.

Orphanides, Athanasios and David Zervos (1995), "Rational Addiction With Learning and Regret”, Journal of Political Economy, 103(4), August, 739-758.

Orphanides, Athanasios and David Zervos, (1998), "Myopia and Addictive Behaviors”, The Economic Journal, 108(446), January, 75-91.

Orzechowski, W. and RC Walker. 2009. The Tax Burden on Tobacco. Historical Compilation 1999, vol. 44. Arlington, Va.

Pacula, R.L., M. Grossman, F.J. Chaloupka, P.M. O’Malley, and M.C. Farrelly. 2001. "Marijuana and Youth.” In J. Gruber (ed.) Risky Behavior Among Youths: An Economic Analysis. (Chicago: University of Chicago Press), pp. 271-326.

Pacula, Rosalie Liccardo. 1998. “Does Increasing the Beer Tax Reduce Marijuana Consumption?” Journal of Health Economics, v. 17, iss. 5, pp. 557-85.

Pampel, Fred C., Patrick M. Krueger, nad Justin T. Denney. 2010. “Socioeconomic Disparities in Health Behaviors.” Annu. Rev. Sociol. 36:347-70.

Perloff, Jeffery M. 2008. Microeconomics. (Addison Wesley: New York).

Petty, N.M., and B. Martin. 2002. "Low-Cost Contingency Management for Treating Cocaineand Opiod-Abusing Metadone Patients.” Journal of Consulting and Clinical Psychology, 70(2): 398-405.

Phelps, E.S. and R.A. Pollack (1968), “On Second-Best National Saving and Game-Equilibrium Growth”, Review of Economic Studies, 35(2), April, 185-199.

Phillips, David P., Nicholas Christenfeld and Natalie M. Ryan (1999), “An Increase in the Number of Deaths in the United States in the First Week of the Month: An Association with Substance Abuse and Other Causes of Death”, New England Journal of Medicine, 341(2), July 8, 93-98.

Philipson, Tomas (1996), "Private Vaccination and Public Health: An Empirical Examination” Journal of Human Resources, 31(3), Summer, 611-630.

Philipson, Tomas. 2000. “Economic Epidemiology and Infectious Diseases.” In A.J. Culyer and J.P Newhouse (eds.) Handbook of Health Economics, Volume 1. (New York: Elsevier), pp. 1539-1628. 
Philipson T, Posner RA. 1999. The long-run growth in obesity as a function of technological change. NBER Working Paper 7423.

Pollak, R.A. (1975). "The intertemporal cost of living index.” Annals of Economic and Social Measurement, 4, 179-95.

Popkin, Barry M., Anna Maria Sieg-Riz and Pamela S. Haines (1996) “A Comparison of Dietary Trends Among Racial and Socioeconomic Groups in the United States”, New England Journal of Medicine, 335(10), September 5, 716-720.

Powell, Lisa M. and Frank J. Chaloupka. 2009. "Food Prices and Obesity: Evidence and Policy Implications for Taxes and Subsidies.” Milbank Quarterly, 87(1): 229-257.

Prendergast, Michael, Deborah Podus, John Finney, Lisa Greenwell, and John Roll. 2006. "Contingency management for treatment of substance use disorders: a meta-analysis." Addiction, 101(11): 1546-1560.

Rachlin, H. (1997). Four teleological theories of addiction. Psychonomic Bulletin and Review, 4(4): 462-473.

Read, D., Loewenstein, G. and Rabin, M. (1999). “Choice Bracketing.” Journal of Risk and Uncertainty. 19:1-3; 171-197.

Register, Charles A. and Donald R. Williams. 1992. "Labor Market Effects of Marijuana and Cocaine Use among Young Men.” Industrial and Labor Relations Review, 45(3): 435448.

Reinhold, Steffan and Hendrik Jurges. 2009. "Secondary School Fees and the Causal Effect of Schooling on Health Behavior.” Health Economics, 19(8): 994-1001.

Renna F. 2007. The economic cost of teen drinking: late graduation and lowered earnings. Health Economics 16: 407-419.

Renna, Francesco, Irina B. Grafova, and Nidhi Thakur. 2008. “The effect of friends on adolescent body weight.” Economics and Human Biology, 6: 377-387.

Rice, Dorothy P., Thomas A. Hodgson, Peter Sinsheimer, Warren Browner and Andrea N. Kopstein. 1986. “The Economic Costs of the Health Effects of Smoking, 1984.” The Milbank Quarterly, 64(4): 489-547.

Rice, Dorothy P., Sander Kelman, and Leonard S. Miller. 1991. "Estimates of economic costs of alcohol and drug abuse and mental illness, 1985 and 1988.” Public Health Rep. 1991 May-Jun; 106(3): 280-292.

Riddell, Chris and Rosemarie Riddell (2006), "Welfare Checks, Drug Consumption, and Health: Evidence from Vancouver Injection Drug Users”, Journal of Human Resources, 41(1), Winter, 138-161.

Roberts, Mark J. and Larry Samuelson (1988), “An Empirical Analysis of Dynamic Nonprice Competition in an Oligopolistic Industry,” Rand Journal of Economics, 19, 200-220.

Rubinstein, Ariel. 2003. "Economics and Psychology? The Case of Hyperbolic Discounting." International Economic Review, 44(4):

Rubinstein, Ariel (2008), “Comments on Neuroeconomics”, Economics and Philosophy, 24(3), November, 485-494.

Rooth, Dan-Olof. 2009. “Obesity, Attractiveness, and Differential Treatment in Hiring: A Field Experiment.” Journal of Human Resources, 44(3): 710-735.

Rosen, Harvey S. 2002. Public Finance, Sixth Edition. (New York: McGraw Hill.)

Rosenzweig, Mark R. and T. Paul Schultz (1989), "Schooling, Information and Nonmarket Productivity” Contraceptive Use and Its Effectiveness”, International Economic Review, 30(2), May, 457-477. 
Roux, Larissa and Cam Donaldson. 2004. "Economics and Obesity: Costing the Problem or Evaluating Solutions?” Obesity Research, 12(2): 173-179.

Rubinstein, Ariel (2003), “'Economics and Psychology'? The Case of Hyperbolic Discounting”, International Economic Review, 44(4), November, 1207-1216.

Rubinstein, Ariel (2008), “Comments on Neuroeconomics”, Economics and Philosophy, 24(3), November, 485-494.

Ruhm, Christopher J. (1995), “Economic Conditions and Alcohol Problems”, Journal of Health Economics 14(5), December, 583-603.

Ruhm, Christopher J. (1996), “Alcohol Policies and Highway Vehicle Fatalities”, Journal of Health Economics, 15(4), August, 435-454.

Ruhm, Christopher J. (2000), “Are Recessions Good For Your Health?”, Quarterly Journal of Economics 115(2), May, 617-650.

Ruhm, Christopher J. (2003), “Good Times Make You Sick”, Journal of Health Economics 22(4), July, 637-658.

Ruhm, Christopher J. (2005a), "Healthy Living in Hard Times”, Journal of Health Economics 24(2), March, 341-363.

Ruhm, Christopher J. (2005b), “Mortality Increases During Economic Upturns”, International Journal of Epidemiology, 34(6), December, 1206-1211.

Ruhm, Christopher J. (2006), “Macroeconomic Conditions, Health and Mortality”, in Andrew M. Jones (ed.) Elgar Companion to Health Economics. Cheltenham, UK: Edward Elgar Publishing, 5-16.

Ruhm, Christopher J. (2007), “A Healthy Economy Can Break Your Heart”, Demography, 44(4), November, 829-848.

Ruhm, Christopher J. (2008), “Macroeconomic Conditions, Health and Government Policy”, in Robert F. Schoeni, James S. House, George A. Kaplan and Harold Pollack (eds.), Making Americans Healthier: Social and Economic Policy as Health Policy: Rethinking America's Approach to Improving Health. New York: Russell Sage Foundation, 173200.

Ruhm, Christopher J. and William E. Black (2002), “Does Drinking Really Decrease in Bad Times?”, Journal of Health Economics 21(4), July, 659-678.

Ruhm, Christopher J. (2010), Understanding Overeating and Obesity. Mimeo, University of North Carolina at Greensboro, April.

Ruhm, Christopher J. (1996), “Alcohol Problems and Highway Vehicle Fatalities”, Journal of Health Economics, 15(4),August, 435-454.

Ryder, Jr., Harl E. and Geoffrey M. Heal. 1973. “Optimal Growth with Intertemporally Dependent Preferences.” The Review of Economic Studies, 40(1): 1-31.

Sabia, Joseph J. 2007. “The Effect of Body Weight on Adolescent Academic Performance.” Southern Economic Journal 2007, 73(4), 871-900.

Saffer, Henry, and Frank J. Chaloupka. 1999. "Demographic Differentials in the Demand for Alcohol and Drugs.” In Frank J. Chaloupka, Michael Grossman, Warren K. Bickel, and Henry Saffer (eds.), The Economic Analysis of Substance Use and Abuse. (Cambridge, MA: National Bureau of Economic Research), pp. 133-156.

Saffer, Henry and Frank Chaloupka. 2000. "The effect fo tobacco advertising bans on tobacco consumption.” Journal of Health Economics, 19: 1117-1137.

Saffer, Henry, and Dhaval Dave. 2006. Alcohol Advertising and Alcohol Consumption by Adolescents. Health Economics 15:617-37. 
Samuelson, Paul (1937), “A Note on the Measurement of Utility”, Review of Economic Studies, 4(2), February, 155-161.

Samwick, A. (1998) Discount rate heterogeneity and social security reform. Journal of Development Economics 57, 117-146.

Schmeiser, Maximilian D. 2009. "Expanding Wallets and Waistlines: The Impact of Family Income on the BMI of Women and Men Eligible for the Earned Income Tax Credit.” Health Economics, 18: 1277-1294.

Schoenbaum, Michael (1997), "Do Smokers Understand the Mortality Effects of Smoking? Evidence from the Health and Retirement Survey” American Journal of Public Health, 87(5), May, 755-759.

Sen, Bisakha. 2003. "Can Beer Taxes Affect Teen Pregnancy? Evidence Based on Teen Abortion Rates and Birth Rates.” Southern Economic Journal, October 2003, v. 70, iss. 2, pp. 328-43.

Sen, Bisakha. 2006. “The Relationship between Beer Taxes, Other Alcohol Policies, and Child Homicide Deaths.” B.E. Journal of Economic Analysis and Policy: Topics in Economic Analysis and Policy, 6(1): 1-17.

Shapiro, Jesse M. (2005), "Is there a Daily Discount Rate? Evidence from the Food Stamp Nutrition Cycle”, Journal of Public Economics, 89(2), Feburary, 303-325.

Shiell A, Gerard K, Donaldson C. 1987. “Cost of illness studies: an aid to decision-making?” Health Policy, 8:317-23.

Shiv, Baba and Alexander Fedorikhin (1999), "Heart and Mind in Conflict: The Interplay of Affect and Cognition in Consumer Decision Making”, Journal of Consumer Research, 26(3), December, 278-292.

Simon, Herbert A. (1984), Models of Bounded Rationality, Volume 1. Cambridge, MA: MIT Press.

Simpson LA, Cooper J. 2009. Paying for Obesity: A Changing Landscape. Pediatrics, 123(Suppl5): S301-S307.

Simpson, Mary Ellen, Mary Serdula, Deborah A. Galuska, Cathleen Gillespie, Ralph Donehoo, Carol Macera and Karin Mack (2003), "Walking Trends Among U.S. Adults: The Behavioral Risk Factor Surveillance System, 1987-2000”, American Journal of Preventive Medicine, 25(2), August, 95-100.

Sirard, John R. and Russell R. Pate (2001), "Physical Activity Assessments in Children and Adolescents” Sports Medicine, 31(6), 439-454.

Sloan, Frank A., Jan Ostermann, Christopher Conover, Donald H. Taylor, Jr. and Gabriel Picone. 2004. The Price of Smoking. MIT Press: Cambridge, MA.

Sloan, Frank A., and Justin G. Trogdon. 2004. "The Impact of the Master Settlement Agreement on Cigarette Consumption.” Journal of Policy Analysis and Management, 23(4): 843-55.

Smith, James P. (2007), "Nature and Causes of Trends in Male Diabetes Prevalence, Undiagnosed Diabetes, and the Socioeconomic Status Health Gradient”, Proceedings of the National Academy of Sciences, 104(33), August 14, 13225-13231.

Smith, V. Kerry, Donald H. Taylor, Jr., Frank A. Sloan, F. Reed Johnson, and William H. Desvousges (2001), “Do Smokers Respond to Health Shocks?” Review of Economics and Statistics, 83(4), November, 675-687.

Stead, L.F., G. Bergson, and T. Lancaster (2008), "Physician Advice for Smoking Cessation (Review)”, Cochrane Database Systematic Review, 2, April 16, Article No. CD000165. 
Stephens, Melvin (2003), “' $3^{\text {rd }}$ of the Month': Do Social Security Recipients Smooth Consumption Between Checks”, American Economic Review, 93(1), March, 406-422.

Stephens, Melvin (2006), "Paycheque Receipt and the Timing of Consumption”, Economic Journal, 116(513), July, 680-701.

Stigler, George J. and Gary S. Becker. (1977) “De Gustibus Non Est Disputandum.” American Economic Review, 67(2): 76-90.

Stiglitz, Joseph E. 1989. "Imperfect Information in the Product Market," Chapter 13 in R. Schmalensee and R.D. Willig (editors), The Handbook of Industrial Organization (New York: Elsevier).

Stock, J.H., J.H. Wright, and M. Yogo. 2002. "A Survey of Weak Instruments and Weak Identification in Generalized Method of Moments." Journal of Business and Economic Statistics 20(4): 518-529.

Strotz, R.H. (1955-1956). “Myopia and Inconsistency in Dynamic Utility Maximization.” Review of Economic Studies. 23(3): 165-180.

Suranovic, Steven M., Robert S. Goldfarb and Thomas C. Leonard (1999), “An Economic Theory of Cigarette Addiction”, Journal of Health Economics, 18(1), January, 1-29.

Terza JV. 2002. Alcohol abuse and employment: a second look. Journal of Applied Econometrics 17: 393-404.

Terza, Joseph V., W. David Bradford, and Clara E. Dismuke. 2008. “The Use of Linear Instrumental Variables Methods in Health Services Research and Health Economics: A Cautionary Note.” Health Services Research, 43:3: 1102-1120.

Thaler, Richard (1981), “Some Empirical Evidence on Dynamic Inconsistency”, Economic Letters, 8(3), 201-207.

Thaler, Richard H. and H.M. Shefrin. 1981. “An Economic Theory of Self-Control.” Journal of Political Economy, 89(2): 392-406.

Thaler, Richard H. and Cass R. Sunstein (2009), Nudge. New York: Penguin Books.

Thomas, Dorothy Swaine (1927), Social Aspects of the Business Cycle. New York: Alfred A. Knopf.

Thaler, Richard H. and Cass R. Sunstein (2009), Nudge. New York: Penguin Books.

Thun, Michael J., Richard Peto, Alan D. Lopez, Jane H. Monaco, S. Jane Henley, Clark W. Heath Jr., and Richard Doll, (1997), "Alcohol Consumption and Mortality Among Middle-Aged and Elderly U.S. Adults”, New England Journal of Medicine, 337(24), December 11, 1705-1714.

Trayhurn, P., Beattie, J.H. 2001. "Physiological role of adipose tissue: white adipose tissue as an endocrine and secretory organ.” Proceedings of the Nutrition Society, 60, 329-339.

Trogdon, Justin G., James Nonnemaker, and Joanne Pais. 2008. "Peer Effects in Adolescent Overweight.” Journal of Health Economics, 27: 1388-1399.

U.S. Department of Health, Education and Human Welfare (1964), Smoking and Health: Report of the Advisory Committee to the Surgeon General of the Public Health Service. Washington, D.C.: U.S. Government Printing Office.

U.S. Department of Health and Human Services. (1990) The Health Benefits of Smoking Cessation: A Report of the Surgeon General. Atlanta Georgia: U.S. Dept. of Health and Human Services, Public Health Service, Centers for Disease Control, Center for Chronic Disease Prevention and Health Promotion, Office on Smoking and Health.

U.S. Department of Health and Human Services (2009), National Healthcare Disparities Report. Rockville, MD: AHRQ Publication No. 10-0004, March. 
Van Ours JC. 2004. A pint a day raises a man's pay; but smoking blows that gain away. Journal of Health Economics 23: 863-886.

Van Ours JC. 2007. The effects of cannabis use on wages of prime-age males. Oxford Bulletin of Economics and Statistics 69(5): 619-634.

Van Ours, Jan C. 1995. "The Price Elasticity of Hard Drugs: The Case of Opium in the Dutch East Indies, 1923-1938.” Journal of Political Economy, 103(2): 261-279.

Variyam JN. Do nutrition labels improve dietary outcomes? Health Econ. 2008;17:695-708.

Variyam, Jayachandran N., and John Cawley. 2006. “Nutrition Labels and Obesity.” NBER Working Paper \#11956.

Viscusi, W. Kip (1990), “Do Smokers Underestimate Risks?” Journal of Political Economy, 98(6), December, 1253-1269.

Vohs, Kathleen, Roy F. Baumeister, Brandon J. Schmeichel, Jean M. Twenge, Noelle M. Nelson, and Dianne M. Tice (2008), "Making Choices Impairs Subsequent Self-Control: A Limited-Resource Account of Decision Making, Self-Regulation, and Active Initiative”, Journal of Personality and Social Psychology, 94(5), May, 883-898.

Volpp, Kevin G., Andrea Gurmankin Levy, David A. Asch, Jesse A. Berlin, John J. Murphy, Angela Gomez, Harold Sox, Jingsan Zhu, and Caryn Lerman (2006), “A Randomized Trial of Financial Incentives for Smoking Cessation”, Cancer Epidemiology, Biomarkers and Prevention, 15(1), January, 12-18.Volpp, K.G., John, L.K., Troxel, A.B., et al. (2008). "Financial Incentive Based Approaches for Weight Loss: A Randomized Trial.” Journal of the American Medical Association. 300(22): 2631-2637.

Volpp, Kevin G., Andrea B. Troxel, Mark V. Pauly, Henry A. Glick, Andrea Puig, David A. Asch, et al. (2009), A Randomized, Controlled Trial of Financial Incentives for Smoking Cessation”, New England Journal of Medicine, 360(7), February 12, 699-709.

Wada, Roy, and Erdal Tekin. 2010. "Body composition and wages,” Economics and Human Biology, 8(2): 242-254.

Wagenaar, Alexander C. and Traci L. Toomey (2002), "Effects of Minimum Drinking Age Laws: Review and Analysis of the Literature from 1960 to 2000”, Journal of Studies on Alcohol Supplement No. 14, 63(2), March, 206-225.

Wagenaar AC, Salois MJ, Komro KA. (2009). Effects of Beverage Alcohol Price and Tax Levels on Drinking: A Meta-Analysis of 1003 Estimates from 112 Studies. Addiction 104: 179-190.

Wagstaff, A. 1986. “The Demand for Health: Some New Empirical Evidence.” Journal of Health Economics, 5: 195-233.

Wallinga, David, Heather Schoonover and Mark Muller. 2009. "Considering the Contribution of US Agricultural Policy to the Obesity Epidemic: Overview and Implications” Journal of Hunger and Nutrition 4(1), January, 3-19.

Wang, Ruqu (2007), “The Optimal Consumption and Quitting of Harmful Addictive Goods”, B.E. Journal of Economic Analysis and Policy, 7 (1), Contributions, Article 15.

Waters, Teresa M., and Frank A. Sloan. 1995. "Why Do People Drink? Tests of the Rational Addiction Model,” Applied Economics, 27: 727-36.

Weatherburn, Don, Craig Jones, Karen Freeman, and Toni Makkai. 2002. "Supply control and harm reduction: lessons from the Australian heroin 'drought'.” Addiction, 98: 83-91.

Wilde, Parke E. and Christine K. Ranney (2000), “The Monthly Food Stamp Cycle: Shopping Frequency and Food Intake Decisions in an Endogenous Switching Regression Framework", American Journal of Agricultural Economics, 82(1), February, 200-213. 
Wisdom, Jessica, Julie S. Downs and George Lowenstein (2010), "Promoting Health Choices: Information versus Convenience”, American Economic Journal: Applied Economics, 2(2), April, 164-178.

Wooldridge JM. 2002. Econometric Analysis of Cross Section and Panel Data. MIT Press: Cambridge, MA.

World Health Organization (2009), Global Health Risks: Mortality and Burden of Disease Attributable to Selected Major Risks (WHO Press, Geneva).

Xu, Xin and Robert Kaester (2010), “The Business Cycle and Health Behaviors”, National Bureau of Economic Research Working Paper No. 15737, February.

Yakusheva, Olga, Kandice Kapinos, and Marianne Weiss. 2009. "The Freshman 15: Evidence of Peer Effects and Environmental Influences from a Natural Experiment.” Workingpaper, Marquette University.

Young, Douglas J. and Thomas W. Likens (2000), “Alcohol Regulation and Auto Fatalities”, International Review of Law and Economics, 20(1), March, 107-126.

Young, Douglas J. and Agnieszka, Bielinska-Kwapisz (2002), “Alcohol Taxes and Beverage Prices”, National Tax Journal, 55(1), March, 57-73.

Young, Douglas J. and Agnieszka, Bielinska-Kwapisz (2006), “Alcohol Prices, Consumption and Traffic Fatalities”, Southern Economic Journal, 72(3), January, 690-703.

Zhang, Feng, Chung L. Huang, Biing-Hwan Lin (2008), "Modeling Fresh Organic Produce Consumption with Scanner Data: A Generalized Double-Hurdle Model Approach”, Agribusiness, 24(4), October, 510-522.

Zhang, Qi and Youfa Wang (2004), "Trends in the Association Between Obesity and Socioeconomic Status in U.S. Adults: 1971 to 2000”, Obesity Research, 12(10), October, 1622-1632.

Zohrabian, Armineh and Tomas J. Philipson. 2010. "External Costs of Risky Health Behaviors Associated with Leading Actual Causes of Death in the U.S.: A Review of the Evidence and Implications for Future Research.” Int. J. Environ. Res. Public Health, 7: 2460-2472. 
Table 3.1.1. U.S. Deaths Related to Modifiable Risk Factors, 1990 and 2000

\begin{tabular}{lcc} 
Cause of Death & $\mathbf{1 9 9 0}$ & $\mathbf{2 0 0 0}$ \\
\hline Tobacco & $400000(19 \%)$ & $435000(18 \%)$ \\
Poor Diet/Physical Inactivity & $300000(14 \%)$ & $365000(15 \%)$ \\
Alcohol Consumption & $100000(5 \%)$ & $85000(4 \%)$ \\
Microbial Agents & $90000(4 \%)$ & $75000(3 \%)$ \\
Toxic Agents & $60000(3 \%)$ & $55000(2 \%)$ \\
Motor Vehicles & $25000(1 \%)$ & $43000(2 \%)$ \\
Fire Arms & $35000(2 \%)$ & $29000(1 \%)$ \\
Sexual Behavior & $30000(1 \%)$ & $20000(1 \%)$ \\
Illicit Drug Use & $20000(1 \%)$ & $17000(1 \%)$ \\
All Modifiable Risks & $1060000(50 \%)$ & $1159000(48 \%)$ \\
\hline
\end{tabular}

Note. Sources: 1990 - McGinnis and Foege (1993); 2000 - Mokdad et al. (2004, 2005). The estimate of deaths due to poor diet and physical inactivity was revised downward from 400,000 in Mokdad et al. (2004) to 365,000 in Mokdad et al. (2005). All other figures in the Year 2000 column are from Mokdad et al. (2004). 
Table 3.1.2. U.S. Deaths Related to Modifiable Risk Factors, 2005

\begin{tabular}{lc}
\hline Cause of Death & $\mathbf{2 0 0 5}$ \\
\hline Tobacco Smoking & 467,000 \\
High Blood Pressure & 395,000 \\
Overweight - Obesity (high BMI) & 216,000 \\
Physical Inactivity & 191,000 \\
High Blood Glucose & 190,000 \\
High LDL Cholesterol & 113,000 \\
High Dietary Salt (sodium) & 102,000 \\
Low Dietary Omega-3 Fatty Acids & 84,000 \\
High Dietary Trans Fatty Acids & 82,000 \\
Alcohol Use & 64,000 \\
Low Intake of Fruits and Vegetables & 58,000 \\
Low Dietary Polyunsaturated Fatty & 15,000 \\
Acids & \\
\hline
\end{tabular}

Note. Source: Danaei et al. (2009). 
Table 3.1.3. Leading Causes of Death and Disability-Adjusted Life Years (DALYs) in High Income Countries

\begin{tabular}{lcc}
\hline Risk Factor & \% of Deaths & \% of DALYs \\
\hline Tobacco Use & 17.9 & 10.7 \\
High Blood Pressure & 16.8 & 6.1 \\
Overweight and Obesity & 8.4 & 6.5 \\
Physical Inactivity & 7.7 & 4.1 \\
High Blood Glucose & 7.0 & 4.9 \\
High Cholesterol & 5.8 & 3.4 \\
Low Fruit \& Vegetable & 2.5 & 1.3 \\
Intake & & \\
Urban Outdoor Air & 2.5 & \\
Pollution & & 6.7 \\
Alcohol Use & 1.6 & 1.5 \\
Occupational Risks & 1.1 & 2.1 \\
Illicit Drugs & & \\
\hline
\end{tabular}

Note. Source: World Health Organization (2009), Tables 1 and 2. Table shows top ten risk factors contributing to deaths or DALYs for countries with 2004 per capita incomes exceeding $\$ 10,066$. A blank entry implies that the specified risk factor is not in the top ten. A given death or DALY may be attributed to multiple risk factors and the risk factors may interact with each other (e.g. obesity may cause high blood pressure). 
Table 3.1.4. Percent of Group with Specified Health Behavior or Risk Factor, 2008

\begin{tabular}{|c|c|c|c|c|c|c|c|c|c|}
\hline Group & Smoker & Obese & $\begin{array}{c}\text { Heavy } \\
\text { Drinker }\end{array}$ & $\begin{array}{c}\text { Binge } \\
\text { Drinker }\end{array}$ & $\begin{array}{c}\text { Physically } \\
\text { Inactive }\end{array}$ & $\begin{array}{l}\text { Mammo- } \\
\text { gram }\end{array}$ & $\begin{array}{l}\text { Colorectal } \\
\text { Screening }\end{array}$ & STD & $\begin{array}{c}\text { Sun } \\
\text { Screen }\end{array}$ \\
\hline Full Sample & 20.6 & 27.4 & 5.5 & 22.7 & 38.2 & 57.8 & 42.3 & 3.0 & 16.9 \\
\hline \multicolumn{10}{|l|}{ Sex } \\
\hline Male & 23.1 & 27.0 & 6.2 & 31.9 & 36.0 & --- & 43.1 & 2.0 & 9.7 \\
\hline Female & 18.3 & 27.8 & 4.9 & 14.2 & 40.3 & 57.8 & 41.6 & 3.9 & 23.7 \\
\hline \multicolumn{10}{|l|}{ Race/Ethnicity } \\
\hline White (non-Hispanic) & 22.0 & 26.2 & 6.5 & 25.1 & 35.2 & 59.8 & 45.8 & 2.6 & 19.6 \\
\hline Black (non-Hispanic) & 21.3 & 36.1 & 3.4 & 14.3 & 47.4 & 59.0 & 37.6 & 4.9 & 5.9 \\
\hline Hispanic & 15.8 & 31.3 & 3.9 & 21.7 & 46.9 & 47.1 & 25.2 & 3.4 & 13.0 \\
\hline \multicolumn{10}{|l|}{ Age (years) } \\
\hline $18-34$ & 23.4 & 22.3 & 6.4 & 34.8 & 31.6 & --- & --- & 4.2 & 15.0 \\
\hline $35-54$ & 23.8 & 30.6 & 6.1 & 24.5 & 36.4 & 56.5 & 24.0 & 1.7 & 18.8 \\
\hline $55-74$ & 16.8 & 32.5 & 4.5 & 10.8 & 43.9 & 75.7 & 59.0 & --- & 18.0 \\
\hline \multicolumn{10}{|l|}{ Education } \\
\hline$<$ High School & 27.5 & 33.3 & 4.9 & 15.7 & 61.9 & 47.4 & 31.9 & 2.3 & 8.3 \\
\hline \multicolumn{10}{|l|}{ Graduate } \\
\hline High School & 27.1 & 33.5 & 5.6 & 20.2 & 48.9 & 58.2 & 40.7 & 2.7 & 13.9 \\
\hline \multicolumn{10}{|l|}{ Graduate/GED } \\
\hline Some College & 22.7 & 30.6 & 5.1 & 22.8 & 35.1 & 58.6 & 44.6 & 2.8 & 18.8 \\
\hline College Graduate & 8.9 & 21.4 & 5.0 & 22.2 & 22.0 & 62.4 & 48.2 & 2.7 & 25.8 \\
\hline \multicolumn{10}{|l|}{ Family Income } \\
\hline$<\$ 35,000$ & 27.6 & 29.2 & 6.0 & 21.1 & 50.1 & 49.9 & 39.3 & 4.5 & 10.9 \\
\hline$\$ 35,000-\$ 74,999$ & 21.4 & 30.4 & 5.5 & 23.0 & 38.6 & 57.8 & 42.4 & 3.0 & 16.0 \\
\hline$\geq \$ 75,000$ & 14.4 & 24.2 & 5.9 & 26.6 & 24.7 & 64.5 & 44.2 & 2.0 & 23.3 \\
\hline
\end{tabular}

Note: Data refer to adults from the 2008 National Health Interview Survey and are weighted so as to be nationally representative. The results for education subgroups refer to individuals aged 25 and older. "Smoker" indicates current smoking and "obese" to having a body mass index of 30 or higher. "Heavy" drinkers refer to males (females) averaging $>14$ ( $>7$ ) drinks per week during the last year and "binge" drinking to persons consuming 5 or more drinks during a single day at least once in the last year. Persons are called "physically inactive" if they engaged in vigorous or moderate physical activity or strength training less than once per week. 
"Mammograms" refer to the last two years for females aged 30 and higher. "Colorectal screening” indicates lifetime prevalence for persons 40 and older. "STDs" indicate sexually transmitted diseases other than HIV/AIDS during the last five years for 18-49 year olds. "Sun Screen” indicates always using sunscreen when outside on warm sunny days for more than one hour. 
Table 3.1.5. Probit Estimates of Health Behaviors, 2008

\begin{tabular}{|c|c|c|c|c|c|c|c|c|c|}
\hline Characteristic & Smoker & Obese & $\begin{array}{c}\text { Heavy } \\
\text { Drinker }\end{array}$ & $\begin{array}{c}\text { Binge } \\
\text { Drinker }\end{array}$ & $\begin{array}{c}\text { Physically } \\
\text { Inactive }\end{array}$ & $\begin{array}{l}\text { Mammo- } \\
\text { gram }\end{array}$ & $\begin{array}{l}\text { Colorectal } \\
\text { Screening }\end{array}$ & STD & $\begin{array}{c}\text { Sun } \\
\text { Screen }\end{array}$ \\
\hline Female & $\begin{array}{l}-.055 \\
(.004)\end{array}$ & $\begin{array}{c}.021 \\
(.006)\end{array}$ & $\begin{array}{l}-.017 \\
(.002)\end{array}$ & $\begin{array}{l}-.176 \\
(.004)\end{array}$ & $\begin{array}{c}.024 \\
(.006)\end{array}$ & --- & $\begin{array}{l}-.011 \\
(.007)\end{array}$ & $\begin{array}{c}.017 \\
(.004)\end{array}$ & $\begin{array}{l}.134 \\
(.006)\end{array}$ \\
\hline Black (non-Hispanic) & $\begin{array}{l}-.054 \\
(.006)\end{array}$ & $\begin{array}{c}.121 \\
(.009)\end{array}$ & $\begin{array}{l}-.023 \\
(.003)\end{array}$ & $\begin{array}{l}-.085 \\
(.005)\end{array}$ & $\begin{array}{c}.075 \\
(.008)\end{array}$ & $\begin{array}{l}.066 \\
(.010)\end{array}$ & $\begin{array}{l}-.014 \\
(.010)\end{array}$ & $\begin{array}{l}.017 \\
(.006)\end{array}$ & $\begin{array}{l}-.113 \\
(.004)\end{array}$ \\
\hline Hispanic & $\begin{array}{l}-.125 \\
(.005)\end{array}$ & $\begin{array}{c}.041 \\
(.009)\end{array}$ & $\begin{array}{l}-.025 \\
(.003)\end{array}$ & $\begin{array}{l}-.051 \\
(.006)\end{array}$ & $\begin{array}{c}.062 \\
(.008)\end{array}$ & $\begin{array}{c}.033 \\
(.011)\end{array}$ & $\begin{array}{l}-.100 \\
(.011)\end{array}$ & $\begin{array}{l}-.001 \\
(.005)\end{array}$ & $\begin{array}{l}-.015 \\
(.006)\end{array}$ \\
\hline Age: 25-34 & $\begin{array}{l}.362 \\
(.014)\end{array}$ & $\begin{array}{c}.128 \\
(.013)\end{array}$ & $\begin{array}{l}.069 \\
(.012)\end{array}$ & $\begin{array}{l}.457 \\
(.017)\end{array}$ & $\begin{array}{l}-.162 \\
(.010)\end{array}$ & $\begin{array}{l}-.519 \\
(.009)\end{array}$ & --- & $\begin{array}{l}.024 \\
(.003)\end{array}$ & $\begin{array}{c}.039 \\
(.010)\end{array}$ \\
\hline Age: $35-54$ & $\begin{array}{c}.309 \\
(.013)\end{array}$ & $\begin{array}{c}.159 \\
(.012)\end{array}$ & $\begin{array}{l}.061 \\
(.010)\end{array}$ & $\begin{array}{c}.295 \\
(.017)\end{array}$ & $\begin{array}{l}-.110 \\
(.010)\end{array}$ & $\begin{array}{l}-.100 \\
(.012)\end{array}$ & $\begin{array}{l}-.382 \\
(.009)\end{array}$ & --- & $\begin{array}{c}.042 \\
(.009)\end{array}$ \\
\hline Age: 55-74 & $\begin{array}{l}.235 \\
(.014)\end{array}$ & $\begin{array}{c}.185 \\
(.012)\end{array}$ & $\begin{array}{l}.044 \\
(.009)\end{array}$ & $\begin{array}{l}.169 \\
(.017)\end{array}$ & $\begin{array}{l}-.050 \\
(.010)\end{array}$ & $\begin{array}{l}.098 \\
(.012)\end{array}$ & $\begin{array}{l}-.036 \\
(.010)\end{array}$ & --- & $\begin{array}{c}.044 \\
(.010)\end{array}$ \\
\hline $\begin{array}{l}\text { High School } \\
\text { Graduate/GED }\end{array}$ & $\begin{array}{l}-.017 \\
(.007)\end{array}$ & $\begin{array}{l}-.001 \\
(.009)\end{array}$ & $\begin{array}{l}-.001 \\
(.004)\end{array}$ & $\begin{array}{l}.021 \\
(.008)\end{array}$ & $\begin{array}{l}-.068 \\
(.008)\end{array}$ & $\begin{array}{l}.076 \\
(.011)\end{array}$ & $\begin{array}{l}.074 \\
(.011)\end{array}$ & $\begin{array}{l}.010 \\
(.007)\end{array}$ & $\begin{array}{c}.055 \\
(.010)\end{array}$ \\
\hline Some College & $\begin{array}{l}-.046 \\
(.006)\end{array}$ & $\begin{array}{l}-.012 \\
(.009)\end{array}$ & $\begin{array}{l}.001 \\
(.005)\end{array}$ & $\begin{array}{l}.030 \\
(.009)\end{array}$ & $\begin{array}{l}-.162 \\
(.007)\end{array}$ & $\begin{array}{l}.102 \\
(.011)\end{array}$ & $\begin{array}{l}.124 \\
(.011)\end{array}$ & $\begin{array}{l}.015 \\
(.008)\end{array}$ & $\begin{array}{l}.101 \\
(.010)\end{array}$ \\
\hline College Graduate & $\begin{array}{l}-.139 \\
(.004)\end{array}$ & $\begin{array}{l}-.087 \\
(.008)\end{array}$ & $\begin{array}{l}-.009 \\
(.004)\end{array}$ & $\begin{array}{l}-.003 \\
(.008)\end{array}$ & $\begin{array}{l}-.223 \\
(.007)\end{array}$ & $\begin{array}{l}.126 \\
(.012)\end{array}$ & $\begin{array}{l}.153 \\
(.012)\end{array}$ & $\begin{array}{l}.020 \\
(.009)\end{array}$ & $\begin{array}{c}.160 \\
(.012)\end{array}$ \\
\hline $\begin{array}{l}\text { Income: } \$ 35,000 \text { - } \\
\$ 74,999\end{array}$ & $\begin{array}{l}-.062 \\
(.005)\end{array}$ & $\begin{array}{c}.005 \\
(.007)\end{array}$ & $\begin{array}{l}-.002 \\
(.003)\end{array}$ & $\begin{array}{c}.009 \\
(.006)\end{array}$ & $\begin{array}{l}-.073 \\
(.006)\end{array}$ & $\begin{array}{l}.087 \\
(.009)\end{array}$ & $\begin{array}{c}.062 \\
(.009)\end{array}$ & $\begin{array}{l}-.011 \\
(.003)\end{array}$ & $\begin{array}{c}.040 \\
(.006)\end{array}$ \\
\hline Income: $\geq \$ 75,000$ & $\begin{array}{l}-.105 \\
(.004)\end{array}$ & $\begin{array}{l}-.040 \\
(.007)\end{array}$ & $\begin{array}{l}-.002 \\
(.004)\end{array}$ & $\begin{array}{l}.028 \\
(.007)\end{array}$ & $\begin{array}{l}-.143 \\
(.007)\end{array}$ & $\begin{array}{l}.132 \\
(.010)\end{array}$ & $\begin{array}{l}.087 \\
(.010)\end{array}$ & $\begin{array}{l}-.019 \\
(.003)\end{array}$ & $\begin{array}{c}.076 \\
(.008)\end{array}$ \\
\hline Baseline & .207 & .296 & .051 & .197 & .405 & .564 & .424 & .032 & .169 \\
\hline
\end{tabular}

Note: The table shows average predicted marginal effects from probit models that control for the specified covariates. Standard errors are in parentheses. Data refer to adults aged 25 and higher from the 2008 National Health Interview Survey. See Table 3 for definitions of the dependent variables. The reference group is non-Hispanic white male high school dropouts aged 75 or higher, with 
family incomes less than $\$ 35,000$. "Baseline” estimates indicate average predicted values for the full sample, with covariates evaluated at their actual values. 
Table 3.2.1: Testing Models of Addiction

\begin{tabular}{|c|c|c|c|}
\hline $\begin{array}{c}\text { Predicted Sign of } \\
\text { Coefficient on: }\end{array}$ & Non-Addictive & Myopic Addiction & Rational Addiction \\
\hline $\mathrm{P}(\mathrm{t})$ & - & - & - \\
\hline $\mathrm{P}(\mathrm{t}-1)$ & 0 & + & + \\
\hline $\mathrm{C}(\mathrm{t}-1)$ & 0 & 0 & + \\
\hline $\mathrm{P}(\mathrm{t}+1)$ & 0 & 0 & + \\
\hline $\mathrm{C}(\mathrm{t}+1)$ & 0 & & + \\
\hline
\end{tabular}

Note: Applies to coefficients from the Chaloupka (1992) empirical model of rational addiction. 
Table 3.2.2. Estimates of Price Elasticity of Demand for Cigarettes

\begin{tabular}{|l|l|c|c|}
\hline \multicolumn{1}{|c|}{ Category } & \multicolumn{1}{|c|}{ Variable } & $\begin{array}{c}\text { Median } \\
\text { Price } \\
\text { Elasticity }\end{array}$ & $\begin{array}{c}\text { Number } \\
\text { of } \\
\text { Estimates }\end{array}$ \\
\hline & & & \\
\hline Elasticity Estimate & Short Run & -0.40 & 368 \\
\hline & Long Run & -0.44 & 155 \\
\hline Aggregation & Country & -0.40 & 335 \\
\hline & State/province & -0.60 & 101 \\
\hline & Individual & -0.39 & 87 \\
\hline Gender & Men & -0.50 & 24 \\
\hline & Women & -0.34 & 15 \\
\hline Age & Adult & -0.32 & 17 \\
\hline & Young Adult & -0.76 & 22 \\
\hline & Teen & -1.43 & 8 \\
\hline
\end{tabular}

Source: Gallet and List (2003), Table 2, column1 
Table 3.2.3. Estimates of Price Elasticity of Demand for Food

\begin{tabular}{|l|c|c|}
\hline \multicolumn{1}{|c|}{ Food Category } & $\begin{array}{c}\text { Mean Price } \\
\text { Elasticity of } \\
\text { Demand }\end{array}$ & $\begin{array}{c}\text { Number of } \\
\text { Estimates }\end{array}$ \\
\hline Food away from home & -0.81 & 13 \\
\hline Soft drinks & -0.79 & 14 \\
\hline Fats/oils & -0.48 & 13 \\
\hline Sweets/sugars & -0.34 & 13 \\
\hline Fruit & -0.70 & 20 \\
\hline Vegetables & -0.58 & 20 \\
\hline
\end{tabular}

Source: Andreyeva et al. (2010), Table 1 
Table 3.2.4A. Estimates of Price Elasticity of Demand for Alcohol

\begin{tabular}{|l|c|c|}
\hline \multicolumn{1}{|c|}{ Consumption } & $\begin{array}{c}\text { Mean Price } \\
\text { Elasticity of } \\
\text { Demand }\end{array}$ & $\begin{array}{c}\text { Number of } \\
\text { Estimates }\end{array}$ \\
\hline All alcohol consumption & -0.51 & 91 \\
\hline Beer & -0.46 & 105 \\
\hline Wine & -0.69 & 93 \\
\hline Distilled spirits & -0.80 & 103 \\
\hline Heavy alcohol use & -0.28 & 10 \\
\hline
\end{tabular}

Source: Wagenaar et al. (2009) 
Table 3.2.4B. Estimates of Price Elasticity of Demand for Alcohol

\begin{tabular}{|l|l|c|c|}
\hline \multicolumn{1}{|c|}{ Category } & \multicolumn{1}{|c|}{ Variable } & $\begin{array}{c}\text { Median } \\
\text { Price } \\
\text { Elasticity }\end{array}$ & $\begin{array}{c}\text { Number } \\
\text { of } \\
\text { Estimates }\end{array}$ \\
\hline & & & \\
\hline Elasticity Estimate & Short Run & -0.518 & 1024 \\
\hline & Long Run & -0.816 & 148 \\
\hline Aggregation & Country & -0.490 & 699 \\
\hline & State/province & -0.671 & 375 \\
\hline & Individual & -0.640 & 87 \\
\hline Gender & Men & -0.509 & 1 \\
\hline & Women & -0.750 & 1 \\
\hline Age & Adult & -0.556 & 22 \\
\hline & Young Adult & -0.386 & 13 \\
\hline & Teen & 1.167 & 1 \\
\hline
\end{tabular}

Source: Gallet (2007), Table 2, column1 
Table 3.2.5 Estimates of Price Elasticity of Demand for Various Illicit Drugs

\begin{tabular}{|l|c|c|l|}
\hline \multicolumn{1}{|c|}{$\begin{array}{c}\text { Addictive } \\
\text { Good }\end{array}$} & $\begin{array}{c}\text { Estimate of Price } \\
\text { Elasticity of } \\
\text { Participation }\end{array}$ & $\begin{array}{c}\text { Estimate of Price } \\
\text { Elasticity of Demand } \\
\text { Conditional on Use }\end{array}$ & \multicolumn{1}{|c|}{ Source } \\
\hline Marijuana & -0.3 & & Pacula et al. (2001) \\
\hline Cocaine & -1.0 & -0.3 to -0.4 & Chaloupka, Grossman, and Tauras (1999) \\
\hline Heroin & -0.89 & & Saffer and Chaloupka (1999) \\
\hline
\end{tabular}


Table 3.2.6. Estimates of Income Elasticity of Demand for Cigarettes

\begin{tabular}{|l|l|c|c|}
\hline \multicolumn{1}{|c|}{ Category } & \multicolumn{1}{|c|}{ Variable } & $\begin{array}{c}\text { Median } \\
\text { Income } \\
\text { Elasticity }\end{array}$ & $\begin{array}{c}\text { Number } \\
\text { of } \\
\text { Estimates }\end{array}$ \\
\hline & & & \\
\hline Elasticity Estimate & Short Run & 0.28 & 295 \\
\hline & Long Run & 0.39 & 80 \\
\hline Aggregation & Country & 0.33 & 341 \\
\hline & State/province & 0.30 & 24 \\
\hline & Individual & 0.06 & 10 \\
\hline Gender & Men & 0.27 & 11 \\
\hline & Women & 1.23 & 8 \\
\hline Age & Adult & 0.06 & 6 \\
\hline & Young Adult & 0.05 & 1 \\
\hline & Teen & - & 0 \\
\hline
\end{tabular}

Source: Gallet and List (2003), Table 2, column 2 
Table 3.2.7. Estimates of Income Elasticity of Demand for Alcohol

\begin{tabular}{|l|l|c|c|}
\hline \multicolumn{1}{|c|}{ Category } & \multicolumn{1}{|c|}{ Variable } & $\begin{array}{c}\text { Median } \\
\text { Income } \\
\text { Elasticity }\end{array}$ & $\begin{array}{c}\text { Number } \\
\text { of } \\
\text { Estimates }\end{array}$ \\
\hline & & & \\
\hline Elasticity Estimate & Short Run & 0.676 & 901 \\
\hline Aggregation & Long Run & 0.860 & 113 \\
\hline & Country & 0.768 & 581 \\
\hline & State/province & 0.572 & 359 \\
\hline Gender & Individual & 0.213 & 74 \\
\hline & Men & 0.193 & 2 \\
\hline Age & Women & 0.120 & 11 \\
\hline & Adult & 0.267 & 30 \\
\hline & Young Adult & 0.328 & 4 \\
\hline & Teen & -0.001 & 2 \\
\hline
\end{tabular}

Source: Gallet (2007), Table 2, column 2 
Figure 3.1.1: Trends in Substance Use, Obesity and Energy Intake

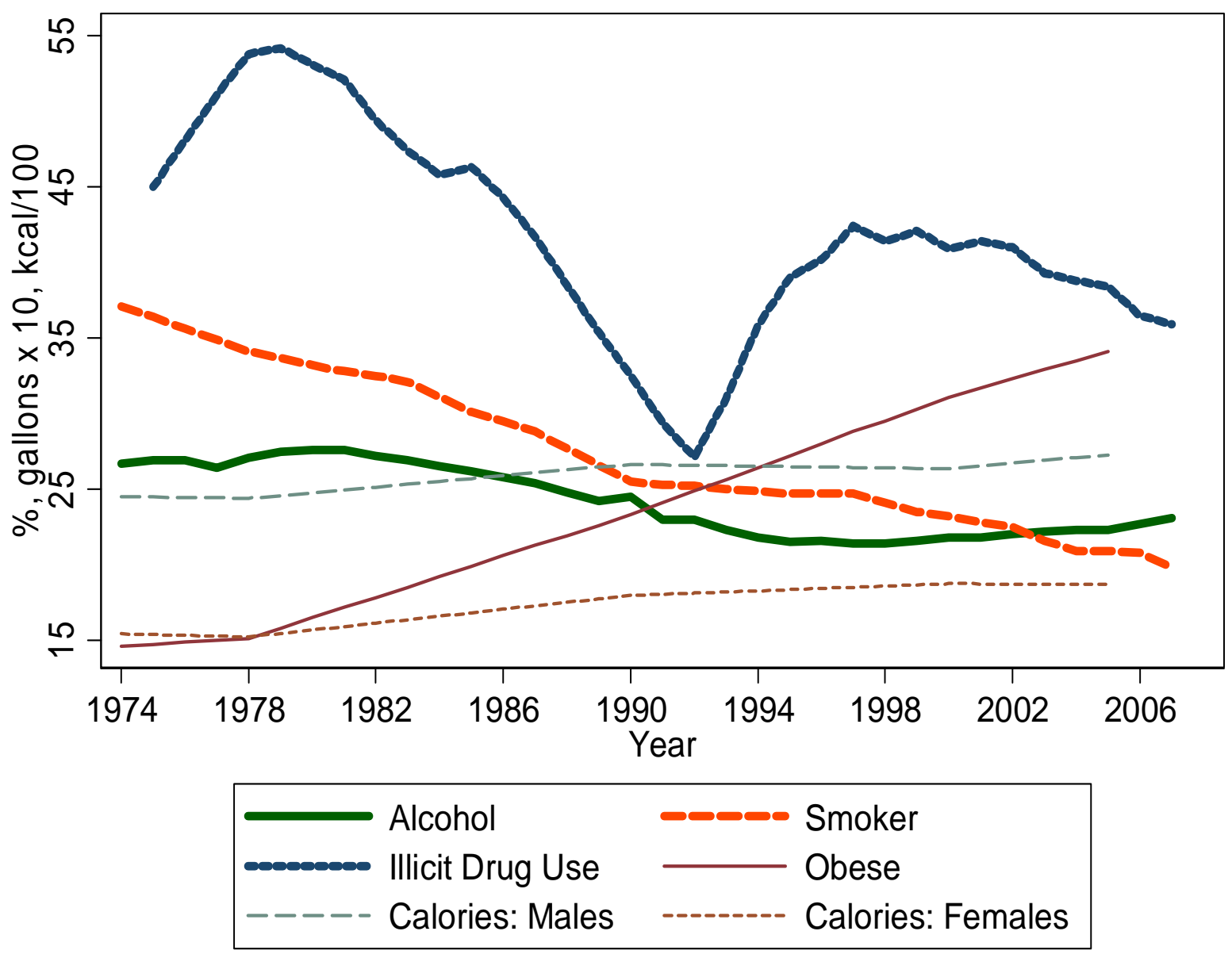

Note: Alcohol refers to apparent per capita ethanol consumption (tenths of gallons) for persons aged $\geq 14$ years (source:

www.niaaa.nih.gov/Resources/DatabaseResources/QuickFacts/AlcoholSales/consum01.htm). Smoker is the percent of adults who are current smokers (source: National Health Interview Survey, www.cdc.gov/tobacco/data_statistics/tables/trends/cig_smoking/index.htm). Illicit drug use indicates use in last year by $12^{\text {th }}$ graders (source: Johnston, et al., 2009). Obesity refers to persons aged $\geq 20$ years with a body mass index $\geq 30$ (source: NCHS, 2010). Calories are average daily energy intake (kcals/100) for persons aged $\geq 20$ years (source: NCHS, 2010). Linear interpolation is used to fill in periods with missing data. The y-axis indicates the percent of the relevant population smoking or who are obese; number of gallons of alcohol consumption $\mathrm{x}$ $1 / 10$, and kcal consumed x 1/100. 
Figure 3.1.2: Trends in Physical Activity, Breastfeeding and Medical Screening

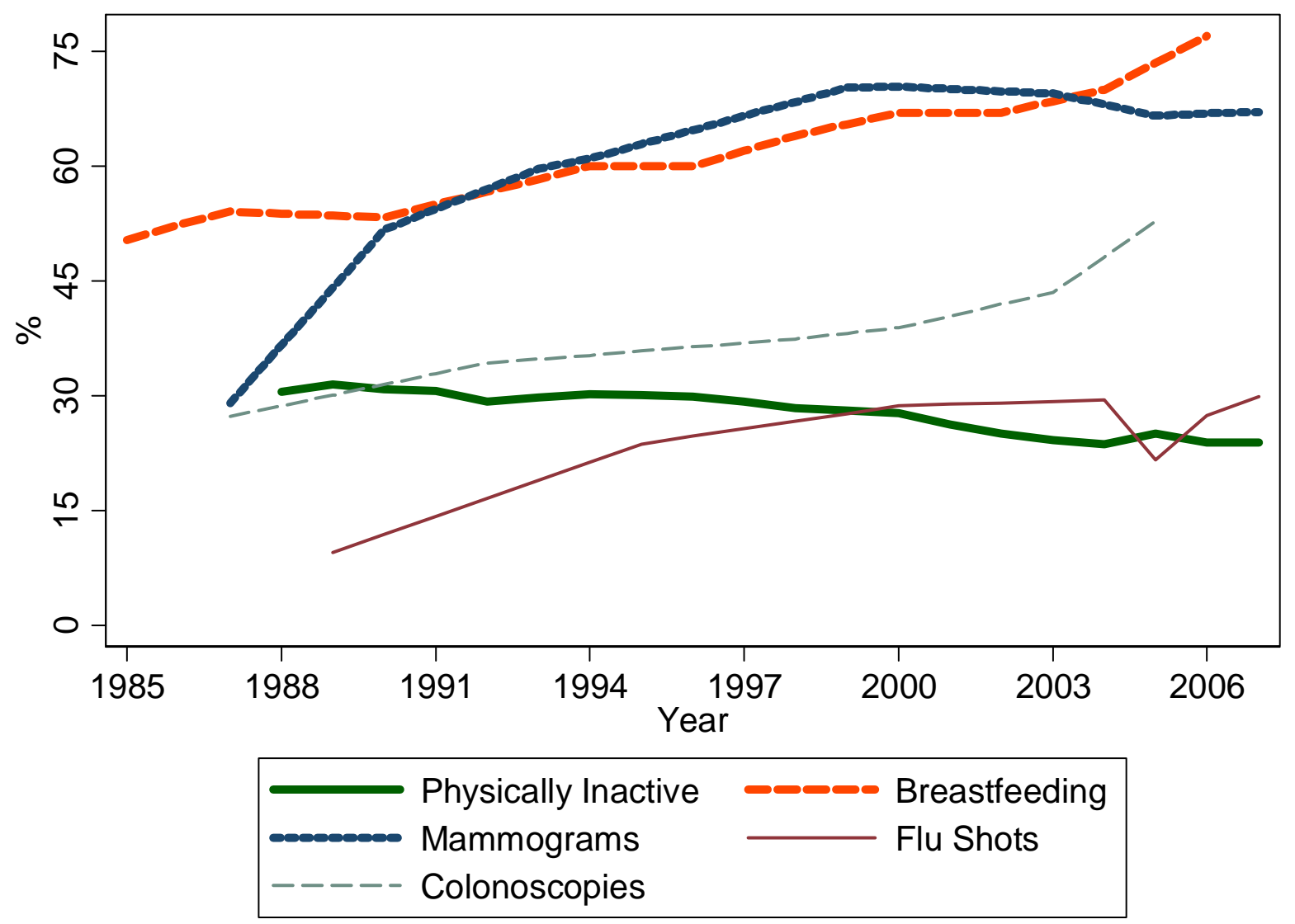

Note: Inactive adults are those reporting no leisure-time physical activity during the last month. Data are for 36 states (source: www.cdc.gov/nccdphp/dnpa/physical/stats/leisure_time.htm). Breastfeeding indicates infants ever breastfed (source: NCHS, 2010; McDowell et al., 2008). Mammograms refer to women aged $\geq 40$ years receiving mammograms in last 2 years. Flu shots refers to percent of adults receiving influenza vaccination in the last 12 months (source, NCHS, 2010). Colonoscopy indicates the percentage of persons aged $\geq 50$ years who have ever had a colonoscopy or sigmoidoscopy (source:

http://progressreport.cancer.gov/doc_detail.asp?pid=0\&did=0\&chid=72\&coid=718\&mid=\#trend s). Linear interpolation is used to fill in periods with missing data. 
Figure 3.1.3: Number of Economics Publications Examining Health Behaviors, 1980-2009

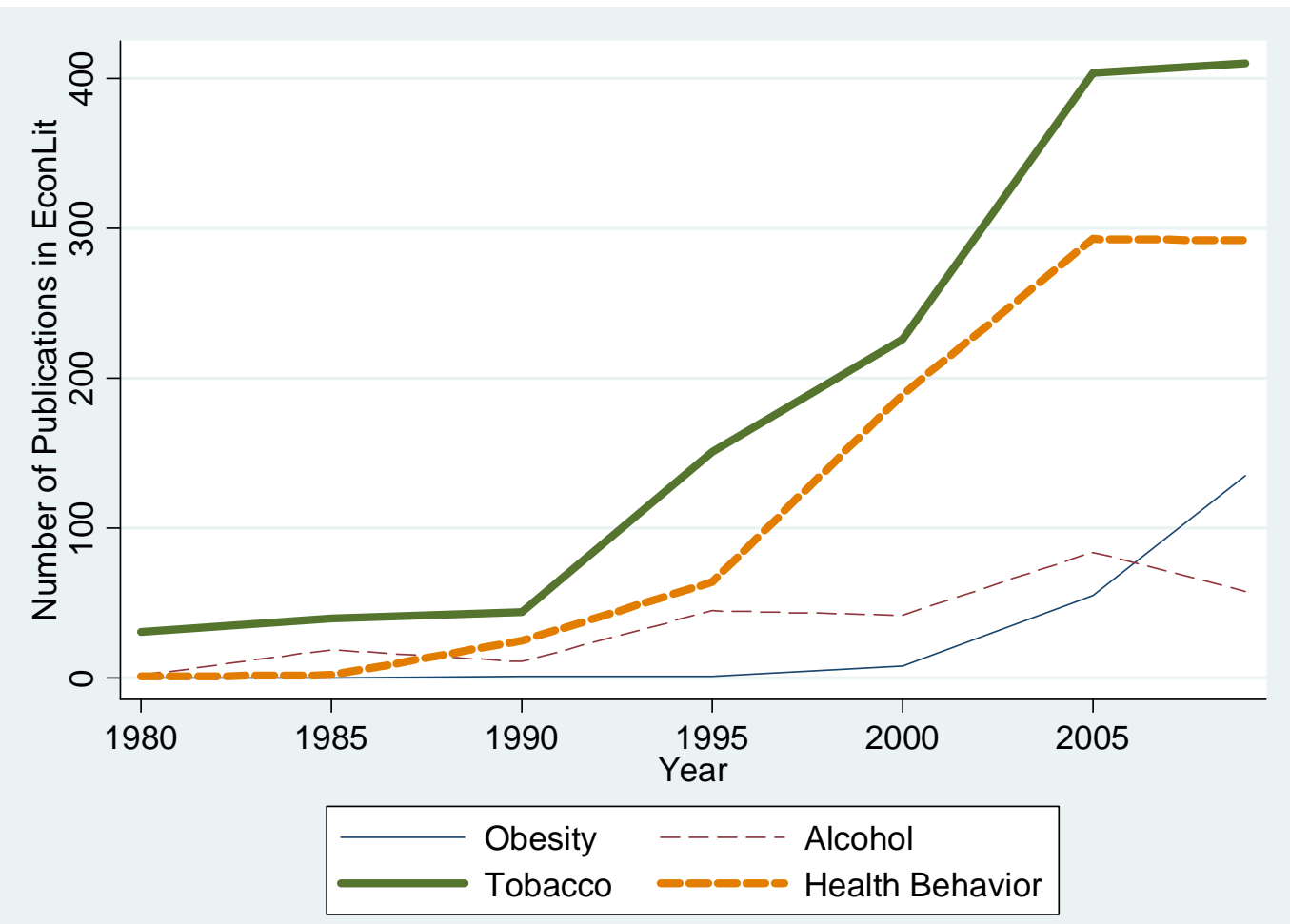

Notes: Figure is based on year-specific searches of EconLit, a database of journal articles, dissertations, and working papers in economics. Obesity indicates the number of publications with the keyword "overweight" or "obesity." Alcohol indicates the number of publications with the keyword "alcohol" or "drinking." Tobacco indicates the number of publications with the keyword "tobacco," "cigarettes," or "smoking." Health behavior indicates the number of publications with the keyword "health behavior." 
Figure 3.2.1: Graphical Representation of Characteristics of Addiction

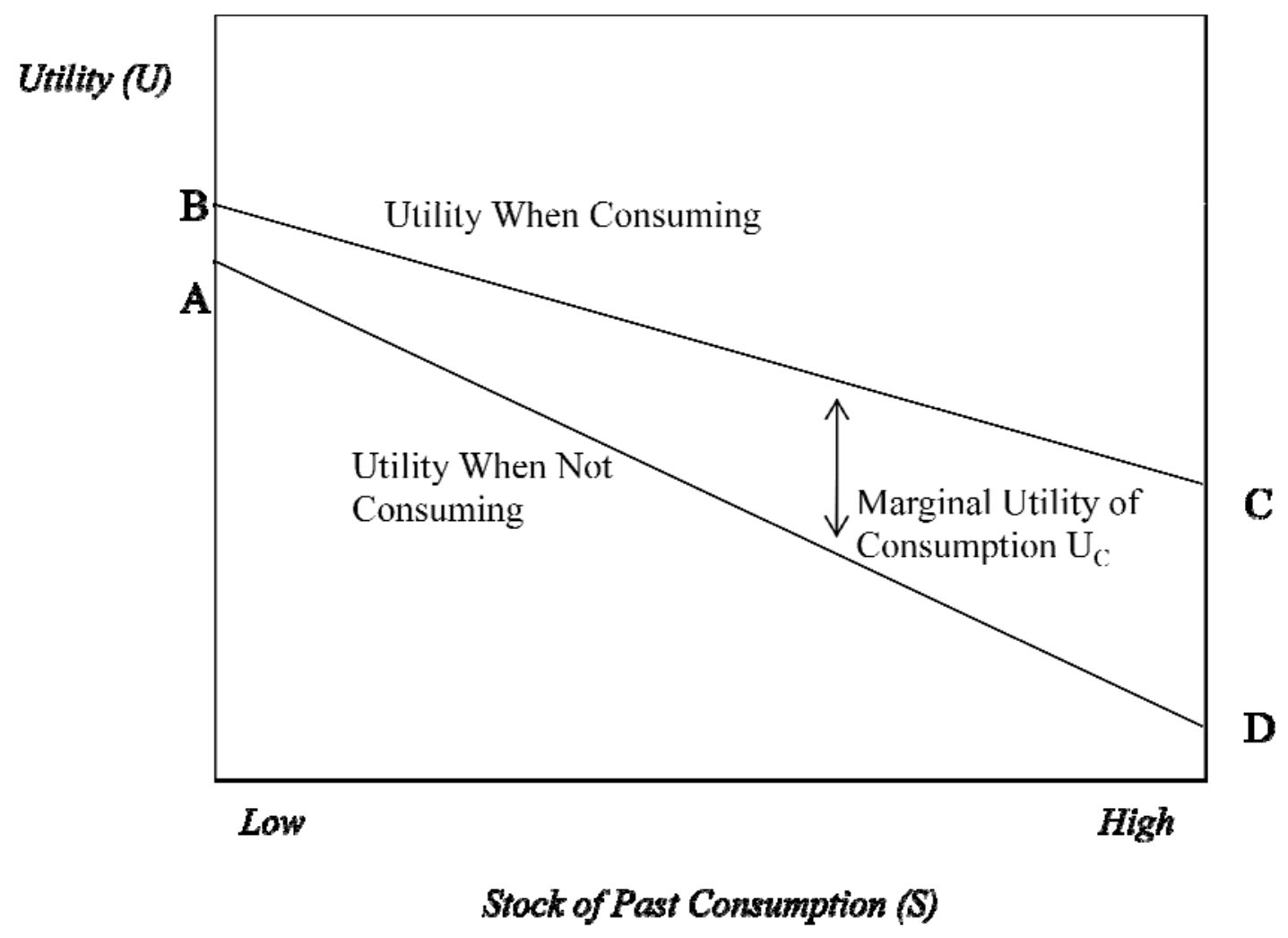

Notes: Adapted from Rachlin (1997). 